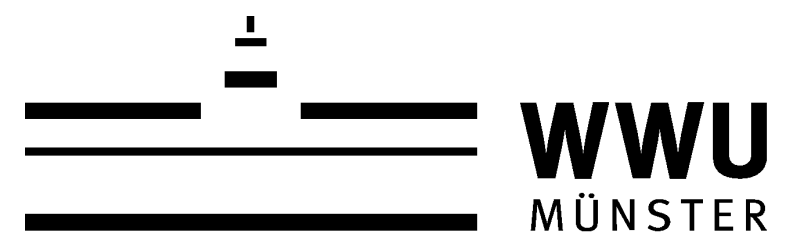

Westfälische Wilhelms-Universität Münster Fachbereich Mathematik und Informatik

André Schemaitat

\title{
THE JIANG-SU ALGEBRA IS STRONGLY SELF-ABSORBING REVISITED
}



Mathematik

Inaugural-Dissertation

zur Erlangung des Doktorgrades

der Naturwissenschaften im Fachbereich

Mathematik und Informatik

der Mathematisch-Naturwissenschaftlichen Fakultät

der Westfälischen Wilhelms-Universität Münster

vorgelegt von

André-Harald Schemaitat

aus Kleve

- 2019 - 
Dekan: Prof. Dr. Xiaoyi Jiang

Erster Gutachter: Prof. Dr. Wilhelm Winter

Zweiter Gutachter: Prof. Dr. Siegfried Echterhoff

Tag der mündlichen Prüfung: 10.02 .2020

Tag der Promotion: 10.02 .2020 


\begin{abstract}
We give a shorter proof of the fact that the Jiang-Su algebra is strongly self-absorbing. This is achieved by introducing and studying so-called unitarily suspended endomorphisms of generalized dimension drop algebras. Along the way we prove uniqueness and existence results for maps between dimension drop algebras and UHF-algebras, using both elementary methods and more advanced tools like the Cuntz semigroup and uniqueness of the hyperfinite $\mathrm{II}_{1}$-factor.
\end{abstract}

\title{
Zusammenfassung
}

In dieser Arbeit präsentieren wir einen kürzeren Beweis dafür, dass die Jiang-Su Algebra stark selbst-absorbierend ist. Als fundamentales Werkzeug definieren wir sogenannte unitär eingehängte Endomorphismen, dessen Existenz aus der Klassifizierung von Abbildungen zwischen Dimension-DropAlgebren und UHF-Algebren folgt. Diese Klassifikation beweisen wir mit elementaren Mitteln, jedoch werden auch alternative Zugänge mithilfe der Cuntz Halbgruppe oder auch der Eindeutigkeit des hyperendlichen $\mathrm{II}_{1}$-Faktors dargelegt. 


\section{Acknowledgements}

First of all I thank my parents and my wife Leonie for their infinite love and support.

I also thank Jacopo, Christian, Federico, Matthias, Shirly, Sabrina, Elizabeth, Zhuofeng, Tim, Timo, Hannes and the whole work group for their company in Münster. A special thank goes to Dominic Enders for always inspiring me.

I also thank everyone within the wonderful operator algebra community, that I met during my travels, and especially those who became friends.

Finally I thank Wilhelm Winter for supervising me and suggesting the topic of this thesis. 


\section{Contents}

Introduction 8

\begin{tabular}{ll}
\hline I Preliminaries & 12
\end{tabular}

I.1 Notation . . . . . . . . . . . . . . . . . . . . . . . . . . . . . . 12

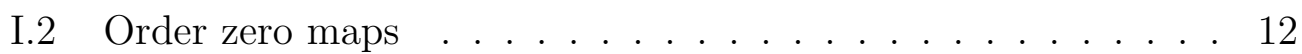

I.3 $\quad$ Generalized dimension drop algebras . . . . . . . . . . . . . 14

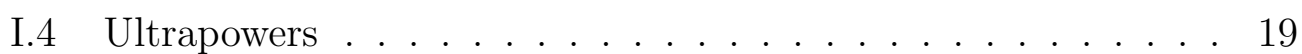

I.5 $\quad$ Some equivalence relations $\ldots \ldots \ldots \ldots$

II Strongly self-absorbing C*-algebras 22

II.1 Generalities . . . . . . . . . . . . . . . . . . . . . . . . . . . . 22

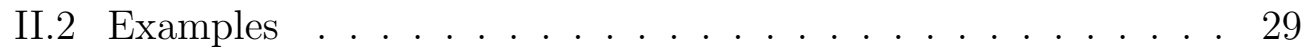

$\begin{array}{ll}\text { III Uniqueness } & 36\end{array}$

III.1 Classification of Lebesgue contractions . . . . . . . . . . . 36

III.2 Uniqueness for order zero maps . . . . . . . . . . . . . . . . . . 39

III.3 Uniqueness for maps on dimension drop algebras. . . . . . . . 41

III.4 Basic Homotopy Lemmas. . . . . . . . . . . . . . . . . . . . 45

III.5 Asymptotic uniqueness . . . . . . . . . . . . . . . . 55

III.6 Uniqueness for endomorphisms of dimension drop algebras . . 57

$\begin{array}{ll}\text { IV Existence } & 59\end{array}$

IV.1 Order zero maps . . . . . . . . . . . . . . . . . . . . . . . . . 59

IV.2 Unitarily suspended endomorphisms . . . . . . . . . . . . . 63

\begin{tabular}{|ll}
\hline A Alternative approaches to classification & 66
\end{tabular}

V.1 The Cuntz semigroup . . . . . . . . . . . . . . . . . . . . . 67

V.2 Uniqueness for order zero maps . . . . . . . . . . . . . . . 74

V.3 Existence of order zero maps . . . . . . . . . . . . . . . . . . 79 
\begin{tabular}{lll}
\hline V.4 Existence and uniqueness of maps on dimension drop algebras & 81
\end{tabular}

\begin{tabular}{|l|l|}
\hline VI Strongly self-absorbing stationary inductive limits & 84
\end{tabular}

VI.1 An approximate intertwining. . . . . . . . . . . . . . . . 84

VI.2 Proof of the main theorem . . . . . . . . . . . . . . . . . . . . 90 


\section{Introduction}

In Elliott's classification program for nuclear $\mathrm{C}^{*}$-algebras, a particularly prominent role is played by strongly self-absorbing $\mathrm{C}^{*}$-algebras (see [Rør02] and Win14]). A separable and unital $\mathrm{C}^{*}$-algebra $D ¥ \mathbb{C}$ is called strongly selfabsorbing if there exists a ${ }^{*}$-isomorphism $D \rightarrow D \otimes D$, which is approximately unitarily equivalent to the first factor embedding $\operatorname{id}_{D} \otimes 1_{D}$ ([TW07]). Strongly self-absorbing $\mathrm{C}^{*}$-algebras have approximately inner flip and hence are simple and nuclear ([ER78]). The list of known strongly self-absorbing $\mathrm{C}^{*}$-algebras is quite short and consists of the Cuntz algebras $\mathcal{O}_{2}, \mathcal{O}_{\infty}$, UHFalgebras of infinite type, tensor products of UHF-algebras of infinite type with $\mathcal{O}_{\infty}$ and the Jiang-Su algebra $\mathcal{Z}$.

The Jiang-Su algebra $\mathcal{Z}$ has been introduced by Jiang and $\mathrm{Su}$ in their remarkable paper [JS99]. For reasons we explain below, it is today one of the most natural and important objects in the classification theory of $\mathrm{C}^{*}$ algebras. Inspired by the work of Connes on the uniqueness of the hyperfinite $\mathrm{II}_{1}$-factor $\mathcal{R}$, Jiang and $\mathrm{Su}$ already observed that $\mathcal{Z}$ is strongly self-absorbing ([JS99, Proposition 8.3, Corollary 8.8]). Before we discuss their proof of this fact in more detail, let us briefly put $\mathcal{Z}$ into more context.

For the classification of $\mathrm{C}^{*}$-algebras, by what is today known as the Elliott invariant, $\mathcal{Z}$-stability is of fundamental importance. The Elliott invariant of a unital $\mathrm{C}^{*}$-algebra $A$ is given by the six-tupel

$$
\left(\mathrm{K}_{0}(A), \mathrm{K}_{0}(A)_{+},\left[1_{A}\right]_{0}, \mathrm{~K}_{1}(A), T(A), r_{A}\right) .
$$

The map $r_{A}$ is a natural pairing between K-theory and the trace simplex $T(A)$. Since $\mathcal{Z}$ has a unique trace and is KK-equivalent to the complex numbers $\mathbb{C}$, in fact it has the same Elliott invariant as $\mathbb{C}$, one might think of $\mathcal{Z}$ as an infinite dimensional version of the complex numbers. Under a natural restriction on the $\mathrm{K}$-groups of a simple $\mathrm{C}^{*}$-algebra $A$, the Elliott invariant of $A$ and $A \otimes \mathcal{Z}$ are isomorphic. This suggests that classification is in general only possible up to $\mathcal{Z}$-stability (cf. [Win14, Definition 2.6]).

Intimately related is the Toms-Winter conjecture (cf. [WZ10, Conjecture 9.3]), which predicts that the following regularity conditions for a (unital) separable, simple, nuclear and infinite dimensional $\mathrm{C}^{*}$-algebra $A$ are equivalent: 
(i) $A$ has finite nuclear dimension,

(ii) $A$ is $\mathcal{Z}$-stable (i.e. $A \cong A \otimes \mathcal{Z}$ ),

(iii) A has strict comparison.

The implication (ii) $\Rightarrow$ (iii) is proven in $[\mathrm{R} \varnothing \mathrm{r} 04$, Corollary 4.6], where the strong self-absorption of $\mathcal{Z}$ is used in an essential way. Just recently, in $\left[\mathrm{CET}^{+} 19\right]$, the nuclear dimension of a $\mathrm{C}^{*}$-algebra like $A$ has been computed to be zero or one in the $\mathcal{Z}$-stable case and infinite otherwise. Together with a result by Winter ([Win12, Corollary 6.3]) this proves the equivalence between (ii) and (i). By previous results of many hands and decades of work, one of the most outstanding results in the classification of $\mathrm{C}^{*}$-algebras follows: unital, separable, simple, nuclear and $\mathcal{Z}$-stable $\mathrm{C}^{*}$-algebras satisfying the UCT are classified by the Elliott invariant $\left(\left[\mathrm{CET}^{+} 19\right.\right.$, Corollary D]). In particular unital, separable, simple and nuclear $\mathrm{C}^{*}$-algebras satisfying the UCT are classified up to $\mathcal{Z}$-stability. The UCT is Rosenberg and Schochet's universal coefficient theorem ([RS87]), which roughly says that the KK-theory can be computed in terms of K-theory. A $\mathrm{C}^{*}$-algebra satisfies the $\mathrm{UCT}$ precisely if it is KK-equivalent to a commutative $\mathrm{C}^{*}$-algebra.

Getting back to the strong self-absorption of $\mathcal{Z}$, let us discuss the proof of Jiang and Su. In [JS99, Section 2] the algebra $\mathcal{Z}$ is constructed as an inductive limit of dimension drop algebras $Z_{p_{n}, q_{n}}$ with $p_{n}, q_{n}$ coprime and tending to infinity, where $Z_{p, q}$ is the $\mathrm{C}^{*}$-algebra of continuous functions $f:[0,1] \rightarrow M_{p} \otimes M_{q}$ such that $f(0) \in M_{p} \otimes 1_{q}$ and $f(1) \in 1_{p} \otimes M_{q}$. Fundamental to their proof that $\mathcal{Z}$ is strongly self-absorbing is a classification machinery for *-homomorphisms between dimension drop algebras ([JS99, Corollary 5.6, Theorem 6.2]). These results rely on a careful analysis of maps between K-homology induced by morphisms between dimension drop algebras.

Although the notion of a strongly self-absorbing $\mathrm{C}^{*}$-algebra was first introduced in [TW07], Jiang and Su already proved two abstract properties of $\mathcal{Z}$, showing that $\mathcal{Z}$ is strongly self-absorbing:

1. $\mathcal{Z}$ has approximately inner half flip, i.e. the first and the second factor embedding of $\mathcal{Z}$ into $\mathcal{Z} \otimes \mathcal{Z}$ are approximately unitarily equivalent,

2. there exists a ${ }^{*}$-homomorphism $\varphi: \mathcal{Z} \otimes \mathcal{Z} \rightarrow \mathcal{Z}$ such that $\varphi \circ\left(\operatorname{id}_{\mathcal{Z}} \otimes 1_{\mathcal{Z}}\right)$ is approximately inner. 
The first property is proven in [JS99, Proposition 8.3], only using basic properties of the construction of $\mathcal{Z}$. The second property is proven in [JS99, Proposition 8.5] and relies heavily on the classification machinery just mentioned. Jiang and Su first show that there exists a ${ }^{*}$-homomorphism $\psi$ from $\mathcal{Z} \otimes \mathcal{Z}$ to $B$, where $B$ is a simple and unital inductive limit of dimension drop algebras having the same Elliott invariant as $\mathcal{Z}$. Now their existence result ([JS99, Theorem 6.2]) provides a unital *-homomorphism $B \rightarrow \mathcal{Z}$. By composing with $\psi$ one gets the desired map $\varphi$. Using that any unital *endomorphism of $\mathcal{Z}$ is approximately inner ([JS99, Theorem 7.6]), it follows that $\varphi \circ\left(\operatorname{id}_{\mathcal{Z}} \otimes 1_{\mathcal{Z}}\right)$ is approximately inner. Here the uniqueness result for maps between dimension drop algebras is used once more.

The goal of this thesis is to prove that the Jiang-Su algebra is strongly self-absorbing in an as self-contained and elementary as possible way. To do so, we use a different picture of $\mathcal{Z}$. Today, there are many descriptions and characterizations of $\mathcal{Z}$, for example as universal $\mathrm{C}^{*}$-algebra ([JW14]) or as the initial object in the category of strongly self-absorbing $\mathrm{C}^{*}$-algebras ([Win11]). For us however, a construction of $\mathcal{Z}$ as an inductive limit of generalized dimension drop algebras will be most suitable. A generalized dimension drop algebra $Z_{\mathfrak{p}, \mathfrak{q}}$ is defined just as before, but now with $M_{p}$ and $M_{q}$ replaced by UHF-algebras $M_{\mathfrak{p}}$ respectively $M_{\mathfrak{q}}$, associated to supernatural numbers $\mathfrak{p}$ and q. More precisely, Rørdam and Winter show in [RW10, Theorem 3.4] that for all coprime supernatural numbers of infinite type $\mathfrak{p}$ and $\mathfrak{q}$, there exists a trace collapsing $\mathbb{1}^{*}$-homomorphism $\mu: Z_{\mathfrak{p}, \mathfrak{q}} \rightarrow Z_{\mathfrak{p}, \mathfrak{q}}$ and that the stationary inductive limit of $Z_{\mathfrak{p}, \mathfrak{q}}$ along $\mu$ is isomorphic to $\mathcal{Z}$, i.e.

$$
Z_{\mathfrak{p}, \mathfrak{q}} \stackrel{\mu}{\longrightarrow} Z_{\mathfrak{p}, \mathfrak{q}} \stackrel{\mu}{\longrightarrow} Z_{\mathfrak{p}, \mathfrak{q}} \stackrel{\mu}{\longrightarrow} \cdots \longrightarrow \mathcal{Z} \text {. }
$$

For classification, this picture of $\mathcal{Z}$ has already been proven to be very useful, most notably in [Win14]. Also the main theorem of [GLN14, Theorem 29.5] relies on this picture of $\mathcal{Z}$. However, showing that the limit of $Z_{\mathfrak{p}, \mathfrak{q}}$ along $\mu$ is strongly self-absorbing still requires comparing it to the original construction of Jiang and Su.

Using the picture of Rørdam and Winter and ideas of Winter (cf. [Win14, Section 4, Definition 4.2]), we introduce so-called unitarily suspended *endomorphisms of generalized dimension drop algebras (Definition IV.2.5). Built into the definition is a unitary path, which in some sense untwists

\footnotetext{
${ }^{1}$ Trace collapsing means that $\tau \circ \mu=\tau^{\prime} \circ \mu$, for all tracial states $\tau$ and $\tau^{\prime}$ on $Z_{\mathfrak{p}, \mathfrak{q}}$.
} 
the trace collapsing ${ }^{*}$-endomorphism. From the definition of a unitarily suspended *-endomorphism, it is then possible to prove that the stationary inductive limit along such a map is strongly self-absorbing. We thus arrive at the following theorem (Theorem IV.2.6. Theorem VI.2.2):

Main Theorem: Let $\mathfrak{p}$ and $\mathfrak{q}$ be coprime supernatural numbers of infinite type. Then,

(i) there exists a unitarily suspended ${ }^{*}$-endomorphism $\rho: Z_{\mathfrak{p q}} \rightarrow Z_{\mathfrak{p}, \mathfrak{q}}$,

(ii) for any unitarily suspended ${ }^{*}$-endomorphism $\sigma$ of $Z_{\mathfrak{p}, \mathfrak{q}}$, the stationary inductive limit

$$
\overrightarrow{Z_{\mathfrak{p}, \mathfrak{q}}^{\sigma}}:=\underline{\lim }\left(Z_{\mathfrak{p}, \mathfrak{q}} \stackrel{\sigma}{\longrightarrow} Z_{\mathfrak{p}, \mathfrak{q}} \stackrel{\sigma}{\longrightarrow} Z_{\mathfrak{p}, \mathfrak{q}} \stackrel{\sigma}{\longrightarrow} \cdots\right)
$$

is strongly self-absorbing.

We will also prove that $\overrightarrow{Z_{\mathfrak{p}, \mathfrak{q}}^{\rho}}$ enjoys all classification relevant properties which $\mathcal{Z}$ has (Theorem VI.2.9 without comparing it to $\mathcal{Z}$. For example, $\overrightarrow{Z_{\mathfrak{p}, \mathfrak{q}}^{\rho}}$ is the initial object in the category of strongly self-absorbing $\mathrm{C}^{*}$-algebras (hence the limit is independent of $\rho$ and the pair $\mathfrak{p}, \mathfrak{q}$ ).

This also confirms that the difficulty of showing that $\mathcal{Z}$ is strongly selfabsorbing lies between that for UHF-algebras of infinite type and $\mathcal{O}_{2}$ or $\mathcal{O}_{\infty}$ (cf. [JW14, 5.2]). Let us also mention that very recently yet another proof of the strong self-absorption of $\mathcal{Z}$ has been found in Gha19, using the theory of Fraïssé limits. This supports the previous statement.

Finally, I want to highlight that in Theorem VI.2.2 no classification theory beyond the classification of UHF-algebras is used. In particular, we do not rely on the classification of morphisms between dimension drop algebras or K-homological results. This is possible by proving existence and uniqueness results for the very specific class of maps between generalized prime dimension drop algebras and certain UHF-algebras. 


\section{Chapter I}

\section{Preliminaries}

\section{I.1 Notation}

We denote by $\mathbb{N}$ the set of natural numbers, by $\mathbb{Z}$ the set of integers and by $\mathbb{C}$ the set of complex numbers. A $\mathrm{C}^{*}$-algebra $A$ is a complex Banach ${ }^{*}$-algebra together with an antilinear involution such that $\left\|a^{*} a\right\|=\|a\|^{2}$, i.e. the $\mathrm{C}^{*}$-identity holds. Usually, $\mathrm{C}^{*}$-algebras will be denoted by the letters $A, B, C, \cdots$. If $A$ is a $\mathrm{C}^{*}$-algebra, we will denote by $A_{+}$the set of positive elements in $A$, by $A_{\leq 1}$ the closed unit ball of $A$, by $A^{+}$the forced unitization of $A$ and by $M(A)$ the multiplier algebra of $A$. A tracial state $\tau$ on $A$ is a positive linear functional of norm one such that $\tau(a b)=\tau(b a)$, for all $a, b \in A$. The set of tracial states on $A$ is denoted by $T(A)$. If $\phi: A \rightarrow B$ is a *-homomorphism, we denote by $\phi^{*}: T(B) \rightarrow T(A)$ the induced map on trace spaces. By $M_{n}$ we denote the $\mathrm{C}^{*}$-algebra of $n \times n$-matrices. For $\varepsilon>0$ and $a, b \in A$ we denote $a \approx_{\varepsilon} b$ if and only if $\|a-b\|<\varepsilon$. If $\varphi, \psi: A \rightarrow B$ are maps and $\mathcal{F} \subseteq A$ is a finite subset, we write $\varphi \approx_{(\mathcal{F}, \varepsilon)} \psi$ if and only if $\|\varphi(a)-\psi(a)\|<\varepsilon$, for all $a \in \mathcal{F}$. If $S, T \subseteq A$, we denote $[S, T]=\{s t-t s: s \in S, t \in T\}$. If not specified otherwise we will denote by $\iota$ the canonical generator of $C_{0}(0,1]$, i.e. $\iota(t)=t$, for $t \in(0,1]$.

\section{I.2 Order zero maps}

In this section we recall some basic facts about order zero maps, which have been defined and studied in [WZ09.

I.2.1 Definition. Let $\phi: A \rightarrow B$ be a linear map. We say that $\phi$ is completely 
positive (c.p.) if

$$
\phi \otimes \operatorname{id}_{M_{n}}: A \otimes M_{n} \rightarrow B \otimes M_{n}
$$

is positive, for all $n \in \mathbb{N}$. After identifying $A \otimes M_{n}$ with $M_{n}(A)$, we see that $\phi$ is c.p. if and only if for each $n \in \mathbb{N}$ the following holds:

$$
M_{n}(A) \ni\left(a_{i j}\right)_{i, j=1}^{n} \geq 0 \quad \Rightarrow \quad M_{n}(B) \ni\left(\phi\left(a_{i j}\right)\right)_{i, j=1}^{n} \geq 0 .
$$

If in addition $\|\phi\| \leq 1$, we say that $\phi$ is completely positive contractive (c.p.c.).

I.2.2 Definition. Let $\phi: A \rightarrow B$ be a c.p. map. We say that $\phi$ has order zero if $\phi$ preserves orthogonality, i.e. $\phi(x y)=0$, for all $x, y \in A$ such that $x y=0$.

The following is a version of Stinespring's dilation theorem for order zero maps.

I.2.3 Theorem. Let $\phi: A \rightarrow B$ be a c.p. order zero map and define $C:=$ $\mathrm{C}^{*}(\phi(A)) \subseteq B$. Then, there exists a positive element $h_{\phi} \in C^{* *}$, with $\left\|h_{\phi}\right\|=$ $\|\phi\|$, and $a^{*}$-homomorphism

$$
\pi_{\phi}: A \rightarrow C^{* *} \cap\left\{h_{\phi}\right\}^{\prime}
$$

such that

$$
\phi(x)=\pi_{\phi}(x) h_{\phi}=h_{\phi} \pi_{\phi}(x) \quad(x \in A) .
$$

If $A$ is unital, then $h_{\phi}=\phi\left(1_{A}\right)$.

I.2.4 Remark. Let $\phi: A \rightarrow B$ be a c.p. order zero map. Using the structure theorem one may define an order zero functional calculus via

$$
f(\phi):=f\left(h_{\phi}\right) \pi_{\phi} \quad\left(f \in C_{0}(0,\|\phi\|]_{+}\right) .
$$

If $\phi$ is c.p.c. and $\|f\| \leq 1$, then $f(\phi)$ is again a c.p.c. order zero map.

I.2.5 Lemma. There exists a bijection between the set of c.p.c. order zero maps from $A$ to $B$ and the set of ${ }^{*}$-homomorphisms from $C_{0}(0,1] \otimes A$ to $B$.

More precisely, let $\phi: A \rightarrow B$ be a c.p.c. order zero map. Then, the associated ${ }^{*}$-homomorphism $\bar{\phi}: C_{0}(0,1] \otimes A \rightarrow B$ is given by

$$
\bar{\phi}(f \otimes a)=f\left(h_{\phi}\right) \pi_{\phi}(a) \quad\left(f \in C_{0}(0,1], a \in A\right) .
$$

where $h_{\phi}$ and $\pi_{\phi}$ are as in Theorem I.2.3. Conversely, if $\varphi: C_{0}(0,1] \otimes A \rightarrow B$ is $a^{*}$-homomorphism, then the associated c.p.c. order zero map $\bar{\varphi}$ is given by

$$
\bar{\varphi}(a)=\varphi(\iota \otimes a) \quad(a \in A) .
$$


I.2.6 Remark. Let $\phi: A \rightarrow B$ be a c.p.c. order zero map. It follows immediately from Lemma I.2.5, that the order zero functional calculus for $\phi$ is given by

$$
f(\phi)(a)=\bar{\phi}(f \otimes a) \quad\left(a \in A, f \in C_{0}(0,1]_{+}\right) .
$$

I.2.7 Lemma. Let $\phi: A \rightarrow B$ be a c.p.c. order zero map, $\tau \in T(B)$ and $f \in C_{0}(0,1]_{+}$. Then $\tau \circ f(\phi)$ is a bounded trace on $A$, i.e. a positive linear functional satisfying the trace property.

The following definitions will be at the heart of our theory.

I.2.8 Definition. Let $h \in A$ be a positive contraction and assume $T(A) \neq \emptyset$. Then $h$ is called a Lebesgue contraction if

$$
\tau(f(h))=\tau_{\text {Leb }}(f) \stackrel{\text { def }}{=} \int_{0}^{1} f(t) d t \quad\left(f \in C_{0}(0,1], \tau \in T(A)\right) .
$$

We also say that $h$ has Lebesgue spectral measure, which refers to the fact that the measure corresponding to the functional $f \mapsto \tau(f)$ is the Lebesgue measure.

I.2.9 Definition. Let $A$ be a $\mathrm{C}^{*}$-algebra such that $T(A) \neq \emptyset . \quad \mathrm{A}^{*}$-homomorphism $\varphi: C_{0}(0,1] \rightarrow A$ is called standard if

$$
\tau \circ \varphi=\tau_{\text {Leb }} \quad(\tau \in T(A)) .
$$

Equivalently, $\operatorname{Im}\left(\varphi^{*}: T(A) \rightarrow T\left(C_{0}(0,1]\right)\right)=\left\{\tau_{\text {Leb }}\right\}$.

I.2.10 Lemma. Let $A$ be a unital $\mathrm{C}^{*}$-algebra and let $\phi: A \rightarrow B$ be a c.p.c. order zero map. Then $h_{\phi}=\phi\left(1_{A}\right)$ is a Lebesgue contraction if and only if $\bar{\phi}$ is standard when restricted to $C_{0}(0,1]$.

\section{I.3 Generalized dimension drop algebras}

A supernatural number $\mathfrak{p}$ is a formal product

$$
\mathfrak{p}=\prod_{p \text { prime }} p^{\nu_{p}(\mathfrak{p})}
$$

where $\nu_{p}(\mathfrak{p}) \in \mathbb{N} \cup\{\infty\}$. Note that every natural number is also a supernatural number. There is an obvious way to define the product of two

\footnotetext{
${ }^{1}$ The restriction map is given by $\operatorname{id}_{C_{0}(0,1]} \otimes 1_{A}: C_{0}(0,1] \rightarrow C_{0}(0,1] \otimes A$.
} 
supernatural numbers and we say that $\mathfrak{p}$ is of infinite type if $\mathfrak{p}^{2}=\mathfrak{p}$ and $\mathfrak{p} \neq 1$. This happens precisely when $\mathfrak{p} \neq 1$ and $\nu_{p}(\mathfrak{p}) \in\{0, \infty\}$, for all primes $p$. If $\left(P_{n}\right)_{n=1}^{\infty}$ is a sequence of natural numbers such that $P_{n}$ divides $P_{n+1}$, for every $n \in \mathbb{N}$, we say that the sequence $\left(P_{n}\right)_{n=1}^{\infty}$ converges to $\mathfrak{p}$ if for every prime number $p$ the following holds:

$$
\sup \left\{\nu \in \mathbb{N}: p^{\nu} \text { divides } P_{n}, \text { for some } n \in \mathbb{N}\right\}=\nu_{p}(\mathfrak{p}) .
$$

We then define the UHF-algebra $M_{\mathfrak{p}}$ by

$$
M_{\mathfrak{p}}:=\lim _{\longrightarrow}\left(M_{P_{n}}, i_{n}\right),
$$

where $i_{n}(x)$ is the block diagonal matrix in $M_{P_{n+1}}$ with $\frac{P_{n+1}}{P_{n}}$ copies of $x$ on the diagonal. It is a basic result in the classification of UHF-algebras that the limit does not depend on the sequence $\left(P_{n}\right)_{n=1}^{\infty}$ and the unital maps $i_{n}$, see for example [RLL00, Section 7.4] and Example II.2.1. Furthermore, we say that $\mathfrak{p}$ and $\mathfrak{q}$ are coprime if

$$
\min \left(\nu_{p}(\mathfrak{p}), \nu_{p}(\mathfrak{q})\right)=0
$$

for all primes $p$.

I.3.1 Definition. ([Win14, Definition 3.1]) Let $\mathfrak{p}$ and $\mathfrak{q}$ be supernatural numbers. We then define

$$
Z_{\mathfrak{p}, \mathfrak{q}}:=\left\{f \in C\left([0,1], M_{\mathfrak{p}} \otimes M_{\mathfrak{q}}\right): \begin{array}{l}
f(0) \in M_{\mathfrak{p}} \otimes 1_{\mathfrak{q}}, \\
f(1) \in 1_{\mathfrak{p}} \otimes M_{\mathfrak{q}}
\end{array}\right\} .
$$

We call $Z_{\mathfrak{p}, \mathfrak{q}}$ a generalized dimension drop algebra. If $\mathfrak{p}$ and $\mathfrak{q}$ are coprime, we say that $Z_{\mathfrak{p}, \mathfrak{q}}$ is a prime dimension drop algebra.

I.3.2 Remark. The center of $Z_{\mathfrak{p}, \mathfrak{q}}$ is given by $C([0,1])$, where we identify

$$
i: C([0,1]) \hookrightarrow Z_{\mathfrak{p}, \mathfrak{q}}: f \mapsto\left(t \mapsto f(t) \cdot 1_{\mathfrak{p}} \otimes 1_{\mathfrak{q}}\right)
$$

Furthermore, this inclusion induces an isomorphism of tracial state spaces (cf. [JS99, Lemma 2.4]):

$$
i^{*}: T\left(Z_{\mathfrak{p}, \mathfrak{q}}\right) \stackrel{\cong}{\rightrightarrows} T(C([0,1])) .
$$

For convenience of the reader, let us also compute the K-theory of prime dimension drop algebras. By $\mathrm{K}_{*}(A)$ we denote the group $\mathrm{K}_{0}(A) \oplus \mathrm{K}_{1}(A)$. Furthermore, we denote $V(A) \rightarrow \mathrm{K}_{0}(A):[p] \mapsto[p]_{0}$, where $V(A)$ is the Murray-von Neumann semigroup. 
I.3.3 Lemma. Let $p, q \in \mathbb{N}$ be coprime. Then $\mathrm{K}_{*}\left(Z_{p, q}\right) \cong \mathrm{K}_{*}(\mathbb{C}) \cong(\mathbb{Z}, 0)$. Furthermore, this isomorphism is unital, i.e. $\left[1_{Z_{p, q}}\right]_{0}$ corresponds to $1 \in \mathbb{Z}$.

Proof. Let us write $Z_{p, q}$ as a pullback:

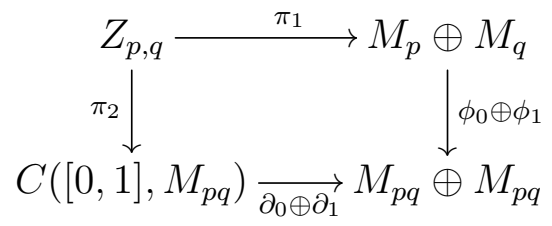

The maps $\partial_{i}$ denote the evaluations at the endpoints. The maps $\phi_{i}$ are given by $\phi_{0}(x)=x \otimes 1_{q}$ and $\phi_{1}(y)=1_{p} \otimes y$. Now, we get a six-term exact Mayer-Vietoris sequence ([Bla98, Theorem 21.2.2]):

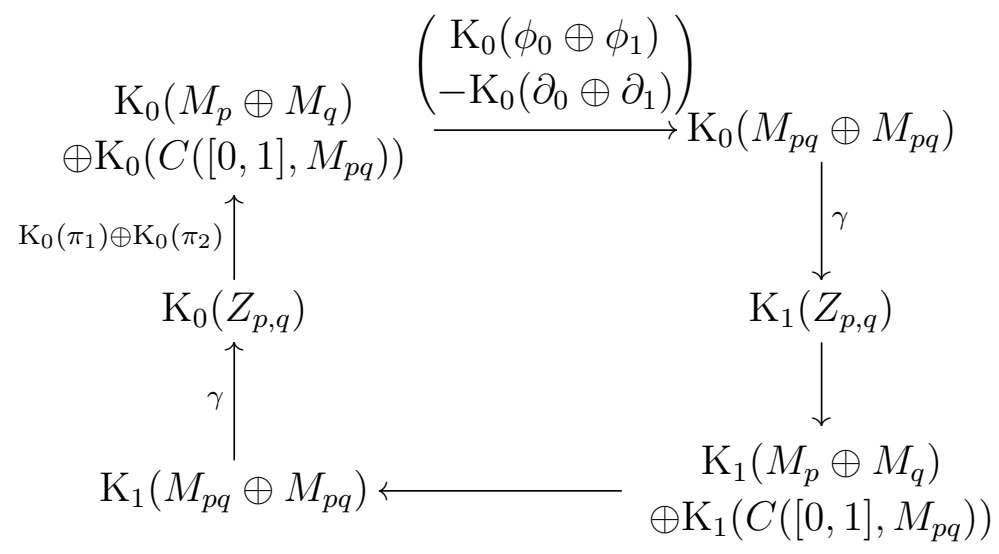

After identifications, this reduces to

$$
0 \longrightarrow \mathrm{K}_{0}\left(Z_{p, q}\right) \longrightarrow \mathbb{Z}^{3} \stackrel{\left(\begin{array}{lll}
q & 0 & -1 \\
0 & p & -1
\end{array}\right)}{\longrightarrow} \mathbb{Z}^{2} \stackrel{\gamma}{\longrightarrow} \mathrm{K}_{1}\left(Z_{p, q}\right) \longrightarrow 0 .
$$

Let us denote the map from $\mathbb{Z}^{3}$ to $\mathbb{Z}^{2}$ by $A$. Then $A$ is surjective, because $p$ and $q$ are coprime. Indeed, we may write $1=\alpha p+\beta q$ for some $\alpha, \beta \in \mathbb{Z}$. Then $A(\beta,-\alpha,-\alpha p)=(1,0)$ and $A(0,0,-1)=(1,1)$. By exactness, it follows immediately that $\gamma=0$ and hence $\mathrm{K}_{1}\left(Z_{p, q}\right)=0$. Next, $\mathrm{K}_{0}\left(Z_{p, q}\right) \cong \operatorname{Ker}(A) \cong$ $\mathbb{Z}$, with generator $(p, q, p q)$. Finally, $\left[1_{Z_{p, q}}\right]_{0} \in \mathrm{K}_{0}\left(Z_{p, q}\right)$ corresponds to the generator $(p, q, p q)$ of $\operatorname{Ker}(A)$, showing that our isomorphism is unital.

I.3.4 Lemma. Let $\mathfrak{p}$ and $\mathfrak{q}$ be coprime supernatural numbers. Then, there is a unital isomorphism

$$
\mathrm{K}_{*}\left(Z_{\mathfrak{p}, \mathfrak{q}}\right) \cong \mathrm{K}_{*}(\mathbb{C})
$$

and $Z_{\mathfrak{p}, \mathfrak{q}}$ is $\mathrm{KK}$-equivalent to $\mathbb{C}$. 
Proof. As in Convention IV.2.3, write $Z_{\mathfrak{p}, \mathfrak{q}}$ as an inductive limit of prime dimension drop algebras. Then, continuity of $K_{*}$ and Lemma I.3.3 show that there is a unital isomorphism $\mathrm{K}_{*}\left(Z_{\mathfrak{p}, \mathfrak{q}}\right) \cong \mathrm{K}_{*}(\mathbb{C})$. Furthermore, note that $Z_{\mathfrak{p}, \mathfrak{q}}$ satisfies the UCT. Indeed, there is an obvious short exact sequence

$$
0 \rightarrow C_{0}(0,1) \rightarrow Z_{\mathfrak{p}, \mathfrak{q}} \rightarrow M_{\mathfrak{p}} \oplus M_{\mathfrak{q}} \rightarrow 0
$$

Since $C_{0}(0,1)$ and $M_{\mathfrak{p}} \oplus M_{\mathfrak{q}}$ satisfy the UCT, a two-out-of-three type of argument ([Rør02, Theorem 2.4 .7 (i)]) shows that $Z_{\mathfrak{p}, \mathfrak{q}}$ satisfies the UCT, too. The statement about KK-equivalence then follows from the fact that the inclusion $\mathbb{C} \hookrightarrow Z_{\mathfrak{p}, \mathfrak{q}}$ defines a KK-equivalence in $\operatorname{KK}\left(\mathbb{C}, Z_{\mathfrak{p}, \mathfrak{q}}\right)$, cf. $[\mathrm{R} \varnothing \mathrm{r} 02$, Theorem 2.4.6 (ii)].

The following definition is a generalization of the one given in $\mathrm{R} \varnothing \mathrm{r} 04$, Section 2].

I.3.5 Definition. Let $\mathfrak{p}$ and $\mathfrak{q}$ be supernatural numbers and let $\varphi: Z_{\mathfrak{p}, \mathfrak{q}} \rightarrow A$ be a unital *-homomorphism, where $A$ is a unital $\mathrm{C}^{*}$-algebra with $T(A) \neq \emptyset$. We say that $\varphi$ is standard if

$$
(\tau \circ \varphi)(f)=\int_{0}^{1} \tau_{M_{\mathfrak{p}} \otimes M_{\mathfrak{q}}}(f(t)) d t \quad\left(\tau \in T(A), f \in Z_{\mathfrak{p}, \mathfrak{q}}\right) .
$$

In other words $\varphi$ is standard if

$$
\tau \circ \varphi=i^{*}\left(\tau_{\text {Leb }}\right) \quad(\tau \in T(A))
$$

where we recall that $i: C([0,1]) \rightarrow Z_{\mathfrak{p}, \mathfrak{q}}$ is the canonical inclusion.

I.3.6 Definition. Let $Z_{\mathfrak{p}, \mathfrak{q}}$ be a generalized dimension drop algebra. We then identify $C_{0}[0,1) \otimes M_{\mathfrak{p}}$ and $C_{0}(0,1] \otimes M_{\mathfrak{q}}$ with subalgebras of $Z_{\mathfrak{p}, \mathfrak{q}}$ via the inclusions

$$
\begin{aligned}
& \grave{\imath}: C_{0}[0,1) \otimes M_{\mathfrak{p}} \hookrightarrow Z_{\mathfrak{p}, \mathfrak{q}} ; \quad \grave{\imath}(f \otimes x)(t)=f(t)\left(x \otimes 1_{\mathfrak{q}}\right), \\
& i: C_{0}(0,1] \otimes M_{\mathfrak{q}} \hookrightarrow Z_{\mathfrak{p}, \mathfrak{q}} ; \quad i(g \otimes y)(t)=g(t)\left(1_{\mathfrak{p}} \otimes y\right) .
\end{aligned}
$$

Whenever $\varphi: Z_{\mathfrak{p}, \mathfrak{q}} \rightarrow A$ is a ${ }^{*}$-homomorphism, we denote

$$
\begin{aligned}
& \grave{\varphi}=\varphi \circ \grave{i} C_{0}[0,1) \otimes M_{\mathfrak{p}} \rightarrow A, \\
& \dot{\varphi}=\varphi \circ \imath: C_{0}(0,1] \otimes M_{\mathfrak{q}} \rightarrow A .
\end{aligned}
$$


Remember, that these maps correspond uniquely to c.p.c. order zero maps

$$
\begin{gathered}
\overline{(\grave{\varphi})^{\prime}}: M_{\mathfrak{p}} \rightarrow A, \\
\overline{\bar{\varphi}}: M_{\mathfrak{q}} \rightarrow A,
\end{gathered}
$$

where $(\cdot)^{\prime}$ means that we have reversed the orientation of the interval. More precisely,

$$
(\grave{\varphi})^{\prime}: C_{0}(0,1] \otimes M_{\mathfrak{p}} \rightarrow A: f \otimes x \mapsto \grave{\varphi}(\check{f} \otimes x),
$$

where $\check{f}(x):=f(1-x)$.

I.3.7 Remark. Note that a ${ }^{*}$-homomorphism $\varphi: Z_{\mathfrak{p}, \mathfrak{q}} \rightarrow A$ is standard if and only if $\varphi \circ i: C([0,1]) \rightarrow A$ is standard. This follows immediately from the fact that $i^{*}$ is an isomorphism (see Remark I.3.2).

I.3.8 Lemma. Let $i$ and $i$ be as in Definition I.3.6. Then the images of $i$ and $i$ commute and

$$
\mathrm{C}^{*}(\operatorname{Im}(\grave{\imath}), \operatorname{Im}(i))=Z_{\mathfrak{p}, \mathfrak{q}},
$$

i.e. $Z_{\mathfrak{p}, \mathfrak{q}}$ is canonically generated by two commuting cones over $M_{\mathfrak{p}}$ resp. $M_{\mathfrak{q}}$. Proof. This follows easily by an approximation argument.

The following theorem by Rørdam and Winter ([RW10, Theorem 2.5]) shows that any prime dimension drop algebra $Z_{p, q}$ can be seen as the universal $\mathrm{C}^{*}$-algebra generated by two c.p.c. order zero maps on $M_{p}$ resp. $M_{q}$, which have commuting ranges and are "large".

I.3.9 Theorem. Let $A$ be a unital $\mathrm{C}^{*}$-algebra and let $p, q \in \mathbb{N}$ be coprime. If $\alpha: M_{p} \rightarrow A$ and $\beta: M_{q} \rightarrow A$ are c.p.c. order zero maps such that

$$
\left[\alpha\left(M_{p}\right), \beta\left(M_{q}\right)\right]=\{0\}, \quad \alpha\left(1_{p}\right)+\beta\left(1_{q}\right)=1_{A},
$$

then there exists a (unique) unital ${ }^{*}$-homomorphism

$$
\varphi: Z_{p, q} \rightarrow A
$$

such that the following diagram commutes:

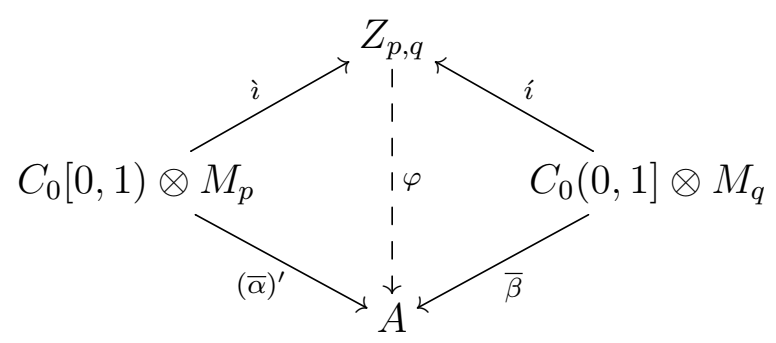


The map $(\bar{\alpha})^{\prime}$ is obtained by reversing the orientation of the unit interval. Furthermore, $\varphi$ is standard if and only if $h_{\alpha}$ and $h^{2} h_{\beta}$ are Lebesgue contractions.

Proof. It remains to prove the last statement. Assume $h_{\beta}$ is a Lebesgue contraction. To show that $\varphi$ is standard, it is enough to show that $\varphi$ is standard on $C([0,1])$, as already observed earlier. Since $C([0,1])$ is the unitization of $C_{0}(0,1]$, it is enough to check that $\varphi$ is standard on $C_{0}(0,1]$. So let $f \in C_{0}(0,1]$ and $\tau \in T(A)$. Then

$$
\tau(\varphi(f))=\tau(\dot{\varphi}(f))=\tau(\bar{\beta}(f))=\tau_{\mathrm{Leb}}(f) .
$$

Note that we use Lemma I.2.10 to show that $\bar{\beta}$ is standard, when restricted to $C_{0}(0,1]$.

Conversely, if $\varphi$ is standard it is easy to check that $h_{\alpha}$ and $h_{\beta}$ are Lebesgue contractions.

\section{I.4 Ultrapowers}

In this thesis we will stick to ultrapowers instead of sequence algebras. The main reason for this is the existence of induced traces on ultrapowers.

I.4.1 Definition. For a fixed free ultrafilter $\omega$ on $\mathbb{N}$, we denote the uniform ultrapower of $A$ by

$$
A_{\omega}:=\ell^{\infty}(A) /\left\{\left(x_{n}\right)_{n=1}^{\infty}: \lim _{n \rightarrow \omega}\left\|x_{n}\right\|=0\right\} .
$$

The equivalence class of a sequence $\left(x_{n}\right)_{n=1}^{\infty} \in \ell^{\infty}(A)$ will be denoted by $\left[\left(x_{n}\right)_{n=1}^{\infty}\right] \in A_{\omega}$. The diagonal inclusion of $A$ into $A_{\omega}$ is denoted by $\iota_{\omega}$. It will be often convenient to identify $A$ in this way with a subalgebra of $A_{\omega}$.

If $\tau \in T(A)$, we get a trace $\tau_{\omega} \in T\left(A_{\omega}\right)$ by

$$
\tau_{\omega}\left(\left[\left(x_{n}\right)_{n=1}^{\infty}\right]\right):=\lim _{\omega} \tau\left(x_{n}\right) .
$$

The limit exists because every bounded sequence of real numbers converges along an ultrafilter. The set of induced traces will be denoted by

$$
T(A)_{\omega}:=\left\{\tau_{\omega}: \tau \in T(A)\right\} \subseteq T\left(A_{\omega}\right) .
$$

\footnotetext{
${ }^{2}$ It is enough to require that $h_{\beta}$ is a Lebesgue contraction, since $h_{\alpha}=1-h_{\beta}$ is then a Lebesgue contraction, too.
} 


\section{I.5 Some equivalence relations}

I.5.1 Definition. Let $A$ and $B$ be $C^{*}$-algebras with $A$ separable and $B$ unital. For ${ }^{*}$-homomorphisms $\varphi, \psi: A \rightarrow B$ we define the following equivalence relations:

(i) Unitary equivalence $\sim_{\mathrm{u}}$ : There exists a unitary $u \in B$ with

$$
u \varphi(a) u^{*}=\psi(a) \quad(a \in A) .
$$

(ii) Approximate unitary equivalence $\approx_{\mathrm{u}}$ : There exists a sequence $\left(u_{n}\right)_{n=1}^{\infty}$ of unitaries in $B$ with

$$
\lim _{n \rightarrow \infty}\left\|u_{n} \varphi(a) u_{n}^{*}-\psi(a)\right\|=0 \quad(a \in A)
$$

(iii) Strong asymptotic unitary equivalence $\sim_{\text {asu }}:$ There exists a unitary path $\left(u_{t}\right)_{t \in[0,1)}$ in $B$ such that $u_{0}=1_{B}$ and

$$
\lim _{t \rightarrow 1}\left\|u_{t} \varphi(a) u_{t}^{*}-\psi(a)\right\|=0 \quad(a \in A) .
$$

(iv) Murray-von Neumann equivalence $\sim$ : There exists a contraction $v \in B$ with

$$
v \varphi(a) v^{*}=\psi(a), \quad v^{*} \psi(a) v=\varphi(a) \quad(a \in A) .
$$

(v) Approximate Murray-von Neumann equivalence $\approx$ : There exists a sequence $\left(v_{n}\right)_{n=1}^{\infty}$ of contractions in $B$ with

$$
\begin{array}{ll}
\lim _{n \rightarrow \infty}\left\|v_{n} \varphi(a) v_{n}^{*}-\psi(a)\right\|=0 & (a \in A), \\
\lim _{n \rightarrow \infty}\left\|v_{n}^{*} \psi(a) v_{n}-\varphi(a)\right\|=0 & (a \in A) .
\end{array}
$$

These equivalence relations also make sense for c.p.c. order zero maps, as they are identified with ${ }^{*}$-homomorphisms on the corresponding cone (see Lemma I.2.5). The same applies to positive contractions, which correspond to c.p.c. order zero maps on $\mathbb{C}$.

Let us also note that our definition of Murray-von Neumann equivalence generalizes the more common Murray-von Neumann equivalence of projections. 
I.5.2 Remark. ([Rør02, Lemma 6.2.5]) Let $A$ and $B$ be $\mathrm{C}^{*}$-algebras with $A$ separable and $B$ unital. For ${ }^{*}$-homomorphisms $\varphi, \psi: A \rightarrow B$ we have

$$
\varphi \approx_{\mathrm{u}} \psi \Longleftrightarrow \iota_{\omega} \circ \varphi \sim_{\mathrm{u}} \iota_{\omega} \circ \psi \Longleftrightarrow \iota_{\omega} \circ \varphi \approx_{\mathrm{u}} \iota_{\omega} \circ \psi
$$

Furthermore, if $\rho, \sigma: A \rightarrow B_{\omega}$ are ${ }^{*}$-homomorphisms, a reindexing argument shows that

$$
\rho \approx_{\mathrm{u}} \sigma \quad \Longleftrightarrow \quad \rho \sim_{\mathrm{u}} \sigma
$$




\section{Chapter II}

\section{Strongly self-absorbing $\mathrm{C}^{*}$-algebras}

In this chapter we recall the concept of a strongly self-absorbing $\mathrm{C}^{*}$-algebra, which was formally introduced and studied by Toms and Winter in [TW07]. Furthermore, we look at the methods used so far to show that $\mathcal{O}_{2}, \mathcal{O}_{\infty}$ and $\mathcal{Z}$ are strongly self-absorbing.

\section{II.1 Generalities}

II.1.1 Definition. Let $D$ be a unital and separable $\mathrm{C}^{*}$-algebra such that $D ¥$ $\mathbb{C}$. Then $D$ is called strongly self-absorbing if there exists an isomorphism $\varphi: D \rightarrow D \otimes D$ such that $\varphi \approx_{\mathrm{u}} \operatorname{id}_{D} \otimes 1_{D}$, i.e. there exists a sequence of unitaries $\left(u_{n}\right)_{n=1}^{\infty}$ in $D \otimes D$ such that

$$
\lim _{n \rightarrow \infty}\left\|u_{n} \varphi(x) u_{n}^{*}-x \otimes 1_{D}\right\|=0 \quad(x \in D) .
$$

II.1.2 Definition. Let $D$ be a unital and separable $\mathrm{C}^{*}$-algebra. We say that $D$ has approximately inner half flip if $\operatorname{id}_{D} \otimes 1_{D} \approx_{\mathrm{u}} 1_{D} \otimes \mathrm{id}_{D}$, i.e. there exists a sequence of unitaries $\left(u_{n}\right)_{n=1}^{\infty}$ in $D \otimes D$ such that

$$
\lim _{n \rightarrow \infty}\left\|u_{n}\left(x \otimes 1_{D}\right) u_{n}^{*}-1_{D} \otimes x\right\|=0 .
$$

Similarly, $D$ has approximately inner flip if the flip map

$$
\sigma_{D, D}: D \otimes D \rightarrow D \otimes D: x \otimes y \mapsto y \otimes x
$$

is approximately inner, i.e. $\sigma_{D, D} \approx_{\mathrm{u}} \mathrm{id}_{D \otimes D}$. 
Let us remark on the difference between approximately inner half flip and approximately inner flip.

II.1.3 Remark. It is clear that approximately inner flip implies approximately inner half flip. The converse however is not true. Examples of $\mathrm{C}^{*}$ algebras having approximately inner half flip but not approximately inner flip are $M_{n+1}\left(\mathcal{O}_{n}\right)$ for $2<n<\infty$, see [FHRT17, Example 3.6].

Another example is the following. Let us look at the unital UCT Kirchberg algebra ${ }^{1} \mathcal{O}^{\infty}$, determined by

$$
\mathrm{K}_{*}\left(\mathcal{O}^{\infty}\right)=(0, \mathbb{Z})
$$

By the Künneth formula, we know that

$$
\mathrm{K}_{*}\left(\mathcal{O}^{\infty} \otimes \mathcal{O}^{\infty}\right)=(\mathbb{Z}, 0)
$$

and the class of the unit is zero, i.e. $\mathcal{O}^{\infty} \otimes \mathcal{O}^{\infty} \cong \mathcal{O}_{\infty}^{\text {st }}$, where st indicates that $\mathcal{O}_{\infty}$ is in standard form ${ }^{2}$. By the classification of Kirchberg algebras, it follows that the half flip of $\mathcal{O}^{\infty}$ is approximately inner, see also [FHRT17, Lemma 3.4] . However, the flip of $\mathcal{O}^{\infty}$ is not approximately inner.

To see this, I refer to an argument ${ }^{3}$ due to Aaron Tikuisis, Dominic Enders and myself: The following diagram commutes, where $A$ and $B$ are arbitrary $\mathrm{C}^{*}$-algebras and $\alpha$ is the natural K-theory product as appearing in the Künneth formula ([B]a98, Theorem 23.1.3]).

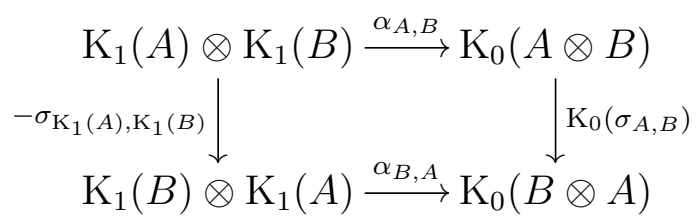

By naturality of $\alpha$ and a diagram chasing argument, the general case can be reduced to $A=B=C(\mathbb{T})$. Let $z \in C(\mathbb{T})$ be the canonical generator, so that $[z]_{1}$ is a generator of $\mathrm{K}_{1}(C(\mathbb{T})) \cong \mathbb{Z}$. We identify $C(\mathbb{T}) \otimes C(\mathbb{T})$ with $C\left(\mathbb{T}^{2}\right)$, and under this identification, the flip map $\sigma_{C(\mathbb{T}), C(\mathbb{T})}$ corresponds to swapping coordinates:

$$
\left(\sigma_{C(\mathbb{T}), C(\mathbb{T})}(f)\right)(w, z)=f(z, w) .
$$

\footnotetext{
${ }^{1} \mathrm{~A}$ Kirchberg algebra is a separable, simple, nuclear and purely infinite $\mathrm{C}^{*}$-algebra.

${ }^{2}$ A unital Kirchberg algebra $A$ is said to be in standard form if $\left[1_{A}\right]_{0}=0$. Equivalently, there exists a unital embedding $\mathcal{O}_{2} \rightarrow A$.

${ }^{3}$ This argument is part of future work and in particular it is a correction of [Tik16. Lemma 4.1].
} 
We have $\mathrm{K}_{0}\left(C\left(\mathbb{T}^{2}\right)\right) \cong \mathbb{Z}^{2}$, generated by $\left[1_{C\left(\mathbb{T}^{2}\right)}\right]_{0}$ and the Bott element $b$. Note that $\mathrm{K}_{0}\left(\sigma_{C(\mathbb{T}), C(\mathbb{T})}\right)(b)=-b^{4}$ and $\alpha_{C(\mathbb{T}), C(\mathbb{T})}\left([z]_{1} \otimes[z]_{1}\right)=b$. Thus,

$$
\begin{aligned}
\alpha_{C(\mathbb{T}), C(\mathbb{T})}\left(\sigma_{\mathrm{K}_{1}(C(\mathbb{T})), \mathrm{K}_{1}(C(\mathbb{T}))}\left([z]_{1} \otimes[z]_{1}\right)\right) & =\alpha_{C(\mathbb{T}), C(\mathbb{T})}\left([z]_{1} \otimes[z]_{1}\right) \\
& =b,
\end{aligned}
$$

and

$$
\begin{aligned}
\mathrm{K}_{0}\left(\sigma_{C(\mathbb{T}), C(\mathbb{T})}\right)\left(\alpha_{C(\mathbb{T}), C(\mathbb{T})}\left([z]_{1} \otimes[z]_{1}\right)\right) & =\mathrm{K}_{0}\left(\sigma_{C(\mathbb{T}), C(\mathbb{T})}\right)(b) \\
& =-b
\end{aligned}
$$

In particular, the following diagram commutes:

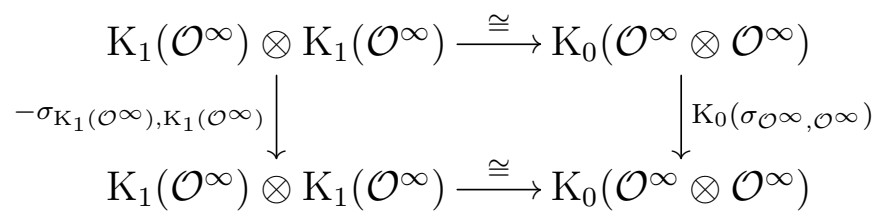

Note that the horizontal map $\alpha_{\mathcal{O}^{\infty}, \mathcal{O}}$ is an isomorphism, by the Künneth formula. After identifying $\mathrm{K}_{1}\left(\mathcal{O}^{\infty}\right) \otimes \mathrm{K}_{1}\left(\mathcal{O}^{\infty}\right)$ with $\mathbb{Z}$, we see that

$$
\mathrm{K}_{0}\left(\sigma_{\mathcal{O}^{\infty}, \mathcal{O}^{\infty}}\right)=-\mathrm{id}
$$

proving that $\sigma_{\mathcal{O}^{\infty}, \mathcal{O}} \infty$ cannot be approximately inner.

Let us recall some standard results about strongly self-absorbing $\mathrm{C}^{*}$ algebras.

II.1.4 Lemma. Let $D$ be a unital and separable $\mathrm{C}^{*}$-algebra. Then the following holds:

(i) if $D$ has approximately inner half flip, then $D$ is simple and nuclear,

(ii) if $D$ is strongly self-absorbing, then $D$ has approximately inner flip and $D$ is either purely infinite or stably finite with a unique trace,

(iii) if $D$ and $E$ are strongly self-absorbing, then $D \otimes E$ is strongly selfabsorbing.

\footnotetext{
${ }^{4}$ Since the Bott element in $\mathrm{K}_{0}\left(C_{0}\left((0,1)^{2}\right)\right)$ arises from a clutching construction, any reflection on $(0,1)^{2}$, and in particular the coordinate swap, i.e., the flip map, sends this Bott element to its inverse. Using the canonical embedding $C_{0}\left((0,1)^{2}\right) \rightarrow C\left(\mathbb{T}^{2}\right)$, it follows that the same is true for the flip map and the Bott element for $C\left(\mathbb{T}^{2}\right)$.
} 
Proof. The proofs of (ii) and (iii) are in [TW07, Section 1]. The proof of (i) is a slight modification of [ER78, Proposition 2.7], where the statement is proven for approximately inner flip. Following their argument, let us just prove that approximately inner half flip implies nuclearity. Given $\mathcal{F} \subseteq D$ finite and $\varepsilon>0$ let $u \in D \otimes D$ be a unitary such that

$$
\left\|u\left(x \otimes 1_{D}\right) u^{*}-1_{D} \otimes x\right\|<\varepsilon \quad(x \in \mathcal{F}) .
$$

Then, find a contraction $v$ in the algebraic tensor product $D \odot D$ such that $\|u-v\|<\frac{\varepsilon}{2}$. Let $\varphi \in S(D)$ be any state on $D$ and consider the slice map ${ }^{5}$

$$
\eta_{\varphi}: D \otimes D \rightarrow D: d \otimes d^{\prime} \mapsto \varphi(d) d^{\prime}
$$

Then $\eta_{\varphi}$ is a c.p.c. map and we get a factorization of $\operatorname{id}_{D}$ on $\mathcal{F}$ up to $\varepsilon$ as follows:

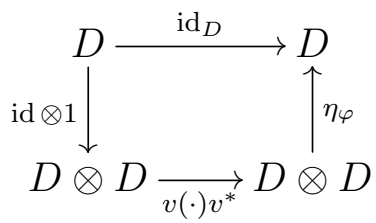

Note that the composition is a finite rank operator ${ }^{6}$ It follows that $D$ is nuclear.

The following is [Rør02, Proposition 7.2.1].

II.1.5 Proposition. Let $A$ and $B$ be separable $\mathrm{C}^{*}$-algebras and assume $\varphi: A \rightarrow B$ is an injective*-homomorphism. Assume that there are unitaries $\left(u_{n}\right)_{n=1}^{\infty}$ in $M(B)_{\omega} \cap \varphi(A)^{\prime}$ such that

$$
\lim _{n \rightarrow \infty} \operatorname{dist}\left(u_{n} b u_{n}^{*}, \varphi(A)_{\omega}\right)=0 \quad(b \in B) .
$$

Then, there is an isomorphism $\psi: A \rightarrow B$, such that $\psi \approx_{\mathrm{u}} \varphi$.

The next theorem is $\mathrm{Rør} 02$, Theorem 7.2.2] and is sometimes referred to as ultrapower intertwining.

II.1.6 Theorem. Let $A$ and $B$ be separable and unital $\mathrm{C}^{*}$-algebras. Assume $B$ has approximately inner half flip and embeds unitally into $A_{\omega} \cap A^{\prime}$. Then, there exists an isomorphism $\varphi: A \rightarrow A \otimes B$ such that $\varphi \approx_{\mathrm{u}} \operatorname{id}_{A} \otimes 1_{B}$. In particular, if $A ¥ \mathbb{C}$ and $A=B$, then $A$ is strongly self-absorbing.

\footnotetext{
${ }^{5}$ The existence of $\eta_{\varphi}$ follows for example from [BO08, Theorem 3.5.3], since $\eta_{\varphi}=$ $\varphi \otimes \operatorname{id}_{D}$

${ }^{6}$ If $v=\sum_{i=1}^{n} x_{i} \otimes y_{i}$, then the image is contained in $\operatorname{span}_{\mathbb{C}}\left\{x_{i} y_{j}^{*}: i, j=1,2, \cdots, n\right\}$.
} 
Proof. We show that the embedding $\operatorname{id}_{A} \otimes 1_{B}: A \rightarrow A \otimes B$ satisfies the hypothesis of Proposition II.1.5. By assumption there exists a unital *-homomorphism $B \rightarrow A_{\omega} \cap A^{\prime}$ and hence also

$$
\alpha: B \rightarrow\left(A \otimes 1_{B}\right)_{\omega} \cap\left(A \otimes 1_{B}\right)^{\prime} \subseteq(A \otimes B)_{\omega} \cap\left(A \otimes 1_{B}\right)^{\prime} .
$$

Denote

$$
\beta: B \rightarrow(A \otimes B)_{\omega} \cap\left(A \otimes 1_{B}\right)^{\prime}: b \mapsto 1_{A} \otimes b .
$$

Note that $\alpha$ and $\beta$ are unital and injective *-homomorphisms with commuting ranges, each of which is isomorphic to $B$. Injectivity of $\alpha$ follows from the fact that $B$ is simple (see Lemma II.1.4 (i)). Hence

$$
\mathrm{C}^{*}(\alpha(B), \beta(B)) \cong B \otimes B
$$

Since $B$ has approximately inner half flip, it follows that there are unitaries $u_{n} \in(A \otimes B)_{\omega} \cap\left(A \otimes 1_{B}\right)^{\prime}$ such that

$$
u_{n} \alpha(b) u_{n}^{*} \rightarrow \beta(b) \quad(b \in B)
$$

Let $a \in A$ and $b \in B$. Then

$$
\begin{aligned}
u_{n}^{*}(a \otimes b) u_{n} & =u_{n}^{*}\left(a \otimes 1_{B}\right) \beta(b) u_{n} \\
& =\left(a \otimes 1_{B}\right) u_{n}^{*} \beta(b) u_{n} \rightarrow\left(a \otimes 1_{B}\right) \alpha(b) \in\left(A \otimes 1_{B}\right)_{\omega} .
\end{aligned}
$$

Hence

$$
\operatorname{dist}\left(u_{n}^{*} x u_{n},\left(A \otimes 1_{B}\right)_{\omega}\right) \rightarrow 0 \quad(x \in A \otimes B) .
$$

By Proposition II.1.5, there exists an isomorphism $\varphi: A \rightarrow A \otimes B$ such that $\varphi \approx_{\mathrm{u}} \mathrm{id}_{A} \otimes 1_{B}$. The last statement follows from the definition of strongly self-absorbing $\mathrm{C}^{*}$-algebras.

The next theorem is [TW07, Proposition 1.10] and provides an abstract way to show that a given $\mathrm{C}^{*}$-algebra is strongly self-absorbing.

II.1.7 Theorem. Let $D$ be a separable and unital $\mathrm{C}^{*}$-algebra such that $D \nsubseteq$ $\mathbb{C}$. Then the following are equivalent:

(i) D is strongly self-absorbing,

(ii) D has approximately inner half flip and there exists a unital *-homomorphism $\psi: D \otimes D \rightarrow D$ such that $\psi \circ\left(\operatorname{id}_{D} \otimes 1_{D}\right) \approx_{\mathrm{u}} \mathrm{id}_{D}$. 
Proof. (ii) $\Rightarrow$ (i): Let $\left(u_{n}\right)_{n=1}^{\infty}$ be a sequence of unitaries in $D \otimes D$ such that

$$
\left\|u_{n} \psi\left(d \otimes 1_{D}\right) u_{n}^{*}-d\right\| \rightarrow 0 \quad(d \in D) .
$$

Define

$$
\phi_{n}: D \rightarrow D: d \mapsto u_{n} \psi\left(1_{D} \otimes d\right) u_{n}^{*} .
$$

Using that $\psi \circ\left(\mathrm{id}_{D} \otimes 1_{D}\right)$ and $\psi \circ\left(1_{D} \otimes \mathrm{id}_{D}\right)$ have commuting ranges, it follows that

$$
\left\|\phi_{n}(d) d^{\prime}-d^{\prime} \phi_{n}(d)\right\| \rightarrow 0 \quad\left(d, d^{\prime} \in D\right)
$$

i.e. $D$ admits an approximately central sequence of unital ${ }^{*}$-homomorphisms. In particular, there exists a unital ${ }^{*}$-homomorphism

$$
D \rightarrow D_{\omega} \cap D^{\prime}: d \mapsto\left[\left(\phi_{n}(d)\right)_{n=1}^{\infty}\right]
$$

Since $D$ has approximately inner half flip, Theorem II.1.6 shows that $D$ is strongly self-absorbing.

(i) $\Rightarrow$ (ii): By assumption, there exists an isomorphism $\varphi: D \rightarrow D \otimes D$ such that $\varphi \approx_{\mathrm{u}} \operatorname{id}_{D} \otimes 1_{D}$. So let $\left(u_{n}\right)_{n=1}^{\infty}$ be a sequence of unitaries in $D \otimes D$ such that

$$
u_{n} \varphi(d) u_{n}^{*} \rightarrow d \otimes 1_{D} \quad(d \in D) .
$$

Define $w_{n}:=\varphi^{-1}\left(u_{n}\right)$ and $\psi:=\varphi^{-1}$. Then

$$
w_{n}^{*} \psi\left(d \otimes 1_{D}\right) w_{n} \rightarrow d \quad(d \in D),
$$

showing that $\psi \circ\left(\operatorname{id}_{D} \otimes 1_{D}\right) \approx_{\mathrm{u}} \operatorname{id}_{D}$. Furthermore, $D$ has approximately inner half flip by Lemma II.1.4 (ii).

II.1.8 Remark. Assuming (ii) in Theorem II.1.7, it is also possible to prove that $D$ is strongly self-absorbing without knowing about ultrapowers or central sequence algebras. Indeed, one can perform an ordinary approximate intertwining, by showing that

$$
\left(\operatorname{id}_{D} \otimes 1_{D}\right) \circ \psi \approx_{\mathrm{u}} \operatorname{id}_{D \otimes D}
$$

The approximate intertwining will then look as follows:

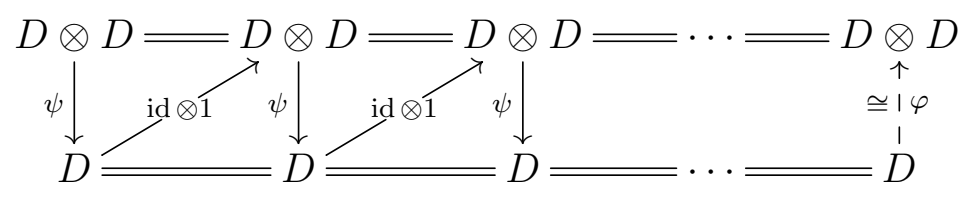


Once this is established, it is entailed in the approximate intertwining that the isomorphism $\varphi: D \rightarrow D \otimes D$ is approximately unitarily equivalent to $\operatorname{id}_{D} \otimes 1_{D}$ (see [Rør02, Corollary 2.3.4]).

Let us denote the factor embeddings by $\imath^{[i]}: D \rightarrow D \otimes D$, for $i=1,2$ (cf. Notation VI.1.1). Using that $D$ has approximately inner half flip, let $\left(\bar{u}_{n}\right)_{n=1}^{\infty}$ be a sequence of unitaries in $D \otimes D$ such that

$$
\left(\bar{u}_{n}\right)^{*}\left(1_{D} \otimes y\right) \bar{u}_{n} \rightarrow y \otimes 1_{D} \quad(y \in D) .
$$

Define an endomorphism $\theta$ of $D \otimes D$ by $\theta=\left(\psi \circ{ }^{[2]}\right) \otimes \operatorname{id}_{D}$ and let $u_{n}:=\theta\left(\bar{u}_{n}\right)$. One then has

$$
\begin{aligned}
u_{n}^{*}\left(1_{D} \otimes y\right) u_{n} & =u_{n}^{*} \theta\left(1_{D} \otimes y\right) u_{n} \\
& \rightarrow \theta\left(y \otimes 1_{D}\right) \\
& =\psi\left(1_{D} \otimes y\right) \otimes 1_{D},
\end{aligned}
$$

for all $y \in D$. Next, by assumption $\psi \circ \imath^{[1]} \approx_{\mathrm{u}} \operatorname{id}_{D}$. Let $\left(v_{n}\right)_{n=1}^{\infty}$ be a sequence of unitaries in $D$ such that

$$
v_{n}^{*} x v_{n} \rightarrow \psi\left(x \otimes 1_{D}\right) \quad(x \in D) .
$$

Define $w_{n}:=\left(v_{n} \otimes 1_{D}\right) u_{n} \in D \otimes D$. Let $\varepsilon>0$ and $x \in D$. Then, for large $n \in \mathbb{N}$ we get:

$$
\begin{aligned}
w_{n}^{*}\left(x \otimes 1_{D}\right) w_{n} & =u_{n}^{*}\left(v_{n}^{*} \otimes 1_{D}\right)\left(x \otimes 1_{D}\right)\left(v_{n} \otimes 1_{D}\right) u_{n} \\
& \approx_{\varepsilon} u_{n}^{*}\left(\psi\left(x \otimes 1_{D}\right) \otimes 1_{D}\right) u_{n} \\
& =\psi\left(x \otimes 1_{D}\right) \otimes 1_{D} .
\end{aligned}
$$

The last equality follows because the images of $\left(\psi \circ \imath^{[1]}\right) \otimes 1_{D}$ and $\theta=(\psi \circ$ $\left.{ }_{\iota^{[2]}}\right) \otimes \mathrm{id}_{D}$ commute. On the other hand, for every $y \in D$, we have:

$$
\begin{aligned}
w_{n}^{*}\left(1_{D} \otimes y\right) w_{n} & =u_{n}^{*}\left(v_{n}^{*} \otimes 1_{D}\right)\left(1_{D} \otimes y\right)\left(v_{n} \otimes 1_{D}\right) u_{n} \\
& =u_{n}^{*}\left(1_{D} \otimes y\right) u_{n} \\
& \rightarrow \psi\left(1_{D} \otimes y\right) \otimes 1_{D} .
\end{aligned}
$$

It follows that

$$
w_{n}^{*}(x \otimes y) w_{n} \rightarrow \psi(x \otimes y) \otimes 1_{D} \quad(x, y \in D) .
$$

This completes the proof. 


\section{II.2 Examples}

In this section we will look at the known examples of strongly self-absorbing $\mathrm{C}^{*}$-algebras and how one proves that these are strongly self-absorbing. In fact, by [TW07, Corollary 5.3], the list of strongly self-absorbing $\mathrm{C}^{*}$-algebras satisfying the UCT consists of the Jiang-Su algebra $\mathcal{Z}$, the Cuntz algebras $\mathcal{O}_{2}, \mathcal{O}_{\infty}$, UHF-algebras of infinite type, denoted by $\mathrm{UHF}^{\infty}$, and tensor products of these. Let us denote the universal UHF-algebra by $\mathcal{Q}$, i.e. $\mathcal{Q}$ is the unique UHF-algebra such that $\left(\mathrm{K}_{*}(\mathcal{Q}),\left[1_{\mathcal{Q}}\right]_{0}\right) \cong(\mathbb{Q}, 0,1)$. These examples follow a certain hierarchy as depicted in the diagram below, where an arrow indicates a unital inclusion:

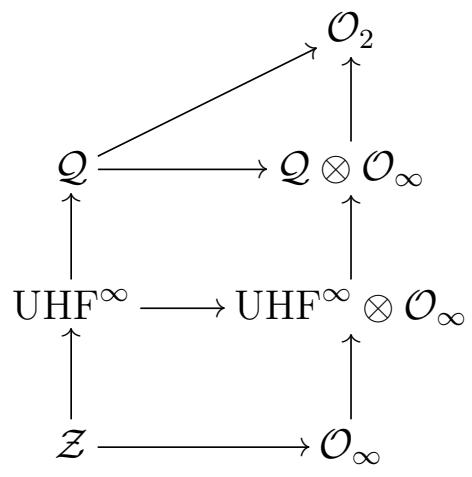

As we do not know any nuclear $\mathrm{C}^{*}$-algebra outside the UCT-class, this list contains all known strongly self-absorbing $\mathrm{C}^{*}$-algebras.

Until now it is only the case of UHF-algebras, where an inductive limit structure is used explicitly to prove strong self-absorption. For all other examples one has to establish some kind of elaborate classification to access the abstract characterization of strongly self-absorbing $\mathrm{C}^{*}$-algebras (Theorem II.1.7). At this point I would like to note that the main theorem of this thesis (Theorem VI.2.2 provides a new strategy to show that the Jiang-Su algebra $\mathcal{Z}$ is strongly self-absorbing by actually using an inductive limit structure.

II.2.1 Example. Let $U$ be a UHF-algebra of infinite type. Then $U$ is strongly self-absorbing. Let us do this for the CAR-algebra

$$
M_{2^{\infty}}=\underset{\lim }{\longrightarrow}\left(M_{2^{n}}, \gamma_{n}\right) \quad \text { with } \quad \gamma_{n}(x)=\left(\begin{array}{ll}
x & 0 \\
0 & x
\end{array}\right) .
$$


We can perform an approximate intertwining

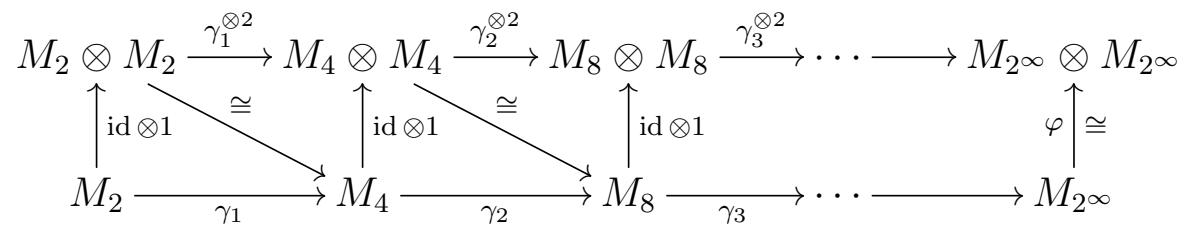

Indeed, it is easy to see that two unital *-homomorphisms from $M_{n}$ to $M_{m}$ must be unitarily equivalent. By [Rør02, Corollary 2.3.3] it then follows that there is an isomorphism $\varphi: M_{2^{\infty}} \rightarrow M_{2^{\infty}} \otimes M_{2^{\infty}}$ and unitaries $\left(u_{n}\right)_{n=1}^{\infty}$ in $M_{2^{\infty}} \otimes M_{2^{\infty}}$ such that

$$
u_{n} \varphi(x) u_{n}^{*} \rightarrow x \otimes 1_{M_{2} \infty} \quad\left(x \in M_{2 \infty}\right) .
$$

Let us check the statement about maps between matrix algebras, since we will come back to it later (in Lemma III.2.1). If $\varphi: M_{n} \rightarrow M_{m}$ is a unital *-homomorphism, then $n \mid m$ and the projections $\varphi\left(e_{i i}\right)$ all have the same rank $\frac{m}{n}$. In particular, if $\psi: M_{n} \rightarrow M_{m}$ is another unital ${ }^{*}$-homomorphism, then $\varphi\left(e_{11}\right)$ and $\psi\left(e_{11}\right)$ are Murray-von Neumann equivalent, i.e. there exists a partial isometry $v \in M_{m}$ such that $\varphi\left(e_{11}\right)=v v^{*}$ and $\psi\left(e_{11}\right)=v^{*} v$. Define

$$
u:=\sum_{i=1}^{n} \varphi\left(e_{i 1}\right) v \psi\left(e_{1 i}\right) .
$$

Then

$$
\begin{aligned}
u u^{*} & =\sum_{i, j=1}^{n} \varphi\left(e_{i 1}\right) v \psi\left(e_{1 i}\right) \psi\left(e_{j 1}\right) v^{*} \varphi\left(e_{1 j}\right)=\sum_{i=1}^{n} \varphi\left(e_{i 1}\right) v v^{*} v v^{*} \varphi\left(e_{1 i}\right) \\
& =\sum_{i=1}^{n} \varphi\left(e_{i 1}\right) v v^{*} \varphi\left(e_{1 i}\right)=\sum_{i=1}^{n} \varphi\left(e_{i i}\right)=1_{M_{m}}
\end{aligned}
$$

Similarly, one computes that $u u^{*}=1_{M_{m}}$ and hence $u$ is a unitary. Furthermore, it is easy to check that

$$
u \psi\left(e_{i j}\right) u^{*}=\varphi\left(e_{i j}\right) \quad(i, j=1,2, \cdots, n) .
$$

It follows that $\varphi \sim_{\mathrm{u}} \psi$.

II.2.2 Example. For the purely infinite examples, let us start with the Cuntz algebra $\mathcal{O}_{2} \cong \mathrm{C}^{*}\left(s_{1}, s_{2}: s_{i}^{*} s_{i}=1, s_{1} s_{1}^{*}+s_{2} s_{2}^{*}=1\right)$. See $\operatorname{Rør} 02$, Chapter 4] for the details. The main tool to study uniqueness is stability of unital *-endomorphisms of purely infinite simple $\mathrm{C}^{*}$-algebras, which is the

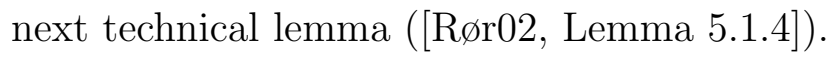


II.2.3 Lemma. Let $A$ be a unital, purely infinite, simple $\mathrm{C}^{*}$-algebra and let $t_{1}, t_{2}$ be isometries in $A$ satisfying $\left.t_{1} t_{1}^{*}+t_{2} t_{2}^{*}=1_{A}\right]^{7}$ Let $\gamma$ be the unital ${ }^{*}$-endomorphism of $A$ given by $\gamma(a)=t_{1} a t_{1}^{*}+t_{2} a t_{2}^{*}$. Then, for each unitary $u \in A$ and for each $\varepsilon>0$, there is a unitary $v \in A$ such that

$$
\left\|v \gamma(v)^{*}-u\right\|<\varepsilon
$$

As a consequence we get the following uniqueness result for $\mathcal{O}_{2}$ :

II.2.4 Theorem. Let $A$ be a unital, purely infinite, simple $\mathrm{C}^{*}$-algebra and let $\varphi, \psi: \mathcal{O}_{2} \rightarrow A$ be unital ${ }^{*}$-homomorphisms. Then $\varphi \approx_{\mathrm{u}} \psi$.

Proof. Let $t_{i}:=\varphi\left(s_{i}\right)$, where $s_{1}, s_{2}$ denote the canonical generators of $\mathcal{O}_{2}$. Define a unitary

$$
u:=\psi\left(s_{1}\right) \varphi\left(s_{1}\right)^{*}+\psi\left(s_{2}\right) \varphi\left(s_{2}\right)^{*} .
$$

By Lemma II.2.3, there exists a sequence $\left(v_{n}\right)_{n=1}^{\infty}$ of unitaries in $A$ such that $v_{n} \gamma\left(v_{n}\right)^{*} \rightarrow u$, where $\gamma(a)=t_{1} a t_{1}^{*}+t_{2} a t_{2}^{*}$. Then, for $i \in \mathbb{Z} / 2 \mathbb{Z}$, the following holds:

$$
\begin{aligned}
v_{n} \varphi\left(s_{i}\right) v_{n}^{*} & =v_{n} t_{i} v_{n}^{*}=v_{n}\left(t_{i} v_{n}^{*} t_{i}^{*}\right) t_{i} \\
& =v_{n}\left(t_{i} v_{n}^{*} t_{i}^{*}+t_{i-1} v_{n}^{*} t_{i-1}^{*}\right) t_{i}=v_{n} \gamma\left(v_{n}\right)^{*} t_{i} \\
& \rightarrow u t_{i}=\psi\left(s_{i}\right) .
\end{aligned}
$$

It follows that $v_{n} \varphi(x) v_{n}^{*} \rightarrow \psi(x)$, for all $x \in \mathcal{O}_{2}$.

II.2.5 Lemma. The Cuntz algebra $\mathcal{O}_{2}$ has approximately inner half flip and admits an asymptotically central sequence of unital ${ }^{*}$-endomorphisms. In particular, $\mathcal{O}_{2}$ embeds unitally into its central sequence algebra $\left(\mathcal{O}_{2}\right)_{\omega} \cap\left(\mathcal{O}_{2}\right)^{\prime}$.

Proof. Since $\mathcal{O}_{2} \otimes \mathcal{O}_{2}$ is purely infinite and simple (cf. Rør02, Theorem 4.1.10]), it follows from Theorem II.2.4 that $\mathcal{O}_{2}$ has approximately inner half flip. For the second statement, note that a sequence $\left(x_{n}\right)_{n=1}^{\infty}$ in $\mathcal{O}_{2}$ is approximately central if and only if $\left\|\lambda\left(x_{n}\right)-x_{n}\right\| \rightarrow 0$, where $\lambda(x)=$ $s_{1} x s_{1}^{*}+s_{2} x s_{2}^{*}$. Indeed, assume that $\left\|\lambda\left(x_{n}\right)-x_{n}\right\| \rightarrow 0$. Let $\varepsilon>0$. For $i=0,1$ and large $n \in \mathbb{N}$ we get:

$$
x_{n} s_{i} \approx_{\varepsilon} \lambda\left(x_{n}\right) s_{i}=\left(s_{1} x_{n} s_{1}^{*}+s_{2} x_{n} s_{2}^{*}\right) s_{i}=s_{i} x_{n} .
$$

\footnotetext{
${ }^{7}$ Which implies that $\mathcal{O}_{2}$ embeds unitally into $A$.
} 
It follows that $\left(x_{n}\right)_{n=1}^{\infty}$ is approximately central. On the other hand, assume $\left(x_{n}\right)_{n=1}^{\infty}$ is approximately central and let $\varepsilon>0$. Then, for large $n \in \mathbb{N}$, we get

$$
\lambda\left(x_{n}\right)=\sum_{i=1}^{2} s_{i} x_{n} s_{i}^{*} \approx_{\varepsilon} x_{n} \sum_{i=1}^{2} s_{i} s_{i}^{*}=x_{n} .
$$

Now, let $u \in \mathcal{O}_{2}$ be a unitary such that $\lambda=\lambda_{u}$, where $\lambda_{u}$ is determined by 8 $\lambda_{u}\left(s_{i}\right)=u s_{i}$. Actually,

$$
u=\sum_{i=1}^{2} \lambda\left(s_{i}\right) s_{i}^{*} .
$$

By Lemma II.2.3 we again find unitaries $\left(v_{n}\right)_{n=1}^{\infty}$ in $\mathcal{O}_{2}$ such that $v_{n} \lambda\left(v_{n}\right)^{*} \rightarrow$ $u$. Define $\rho_{n}:=\lambda_{v_{n}}$. We claim that the $\rho_{n}$ are approximately central. Indeed,

$$
\begin{aligned}
\left\|\lambda\left(\rho_{n}\left(s_{i}\right)\right)-\rho_{n}\left(s_{i}\right)\right\| & =\left\|\lambda\left(v_{n} s_{i}\right)-v_{n} s_{i}\right\|=\left\|\lambda\left(s_{i}\right)-\lambda\left(v_{n}\right)^{*} v_{n} s_{i}\right\| \\
& =\left\|u s_{i}-\lambda\left(v_{n}\right)^{*} v_{n} s_{i}\right\| \\
& \rightarrow\left\|u s_{i}-u s_{i}\right\|=0 .
\end{aligned}
$$

By our previous observation, it follows that $\left(\rho_{n}(x)\right)_{n=1}^{\infty}$ is approximately central, for every $x \in \mathcal{O}_{2}$. Furthermore, the $\rho_{n}$ induce a unital *-homomorphism

$$
\mathcal{O}_{2} \rightarrow\left(\mathcal{O}_{2}\right)_{\omega} \cap\left(\mathcal{O}_{2}\right)^{\prime}: x \mapsto\left[\left(\rho_{n}(x)\right)_{n=1}^{\infty}\right]
$$

By Theorem II.1.6 it now follows that $\mathcal{O}_{2}$ is strongly self-absorbing.

II.2.6 Example. The next purely infinite example is

$$
\mathcal{O}_{\infty}=\mathrm{C}^{*}\left(\left(s_{i}\right)_{i=1}^{\infty}: s_{i}^{*} s_{j}=\delta_{i j} \cdot 1\right)
$$

which behaves quite differently from $\mathcal{O}_{2}$. Let us briefly recall the two main ingredients for this case. As for $\mathcal{O}_{2}$, there is a uniqueness result for $\mathcal{O}_{\infty}$ ( $\left[\mathrm{R} \varnothing \mathrm{r} 02\right.$, Proposition 7.2.5]), showing that $\mathcal{O}_{\infty}$ has approximately inner half flip. The other main ingredient is that the central sequence algebra of a unital Kirchberg algebra is unital, simple and purely infinite ( $\mid \mathrm{R} \varnothing \mathrm{r} 02$, Proposition 7.1.1]). The proof relies on a detailed study of nuclear maps between purely infinite $\mathrm{C}^{*}$-algebras.

\footnotetext{
${ }^{8}$ The map $\lambda_{u}$ exists by the universal property of $\mathcal{O}_{2}$.
} 
II.2.7 Theorem. Let $A$ be a unital Kirchberg algebra. Then $A_{\omega} \cap A^{\prime}$ is a unital, purely infinite and simple $\mathrm{C}^{*}$-algebra.

Applying this theorem to $\mathcal{O}_{\infty}$, we see that the unit of the central sequence algebra of $\mathcal{O}_{\infty}$ is properly infinite, showing that there exists a unital embedding $\mathcal{O}_{\infty} \rightarrow\left(\mathcal{O}_{\infty}\right)_{\omega} \cap\left(\mathcal{O}_{\infty}\right)^{\prime}$, see [Rør02, Proposition 1.1.2]. Again, by Theorem II.1.6, it follows that $\mathcal{O}_{\infty}$ is strongly self-absorbing.

II.2.8 Example. Our final example will be the Jiang-Su algebra $\mathcal{Z}$. In [JS99, $\mathcal{Z}$ is constructed as an inductive limit

$$
\mathcal{Z}=\left(Z_{p_{n}, q_{n}}, \phi_{n}\right)
$$

where $p_{n}, q_{n} \in \mathbb{N}$ are coprime, finite and $p_{n}, q_{n} \rightarrow \infty$. The connecting maps $\phi_{m, n}$ have a particularly explicit form and look like

$$
\phi_{m, n}(f)=u \operatorname{diag}\left(f \circ \xi_{1}, f \circ \xi_{2}, \cdots, f \circ \xi_{k}\right) u^{*} \quad(m<n),
$$

where $u$ is a unitary path in $M_{p_{n} q_{n}}$ and each $\xi_{i}:[0,1] \rightarrow[0,1]$ is a continuous function looking almost constant for larger $n$, more precisely:

$$
\sup _{x, y \in[0,1]}\left|\xi_{i}(x)-\xi_{i}(y)\right| \leq \frac{1}{2^{n-m}} \quad(i=1,2, \cdots, k) .
$$

In this picture, one can prove that $\mathcal{Z}$ is strongly self-absorbing by using Theorem II.1.7. This uses the full power of classification (existence and uniqueness) for unital and simple inductive limits of dimension drop algebras. Let us start with the following existence theorem ([JS99, Theorem 6.2]):

II.2.9 Theorem. Let $A$ and $B$ be unital, infinite dimensional and simple inductive limits of finite direct sums of dimension drop algebra9. If there exists a unital morphism

$$
\alpha: \operatorname{Ell}(A) \rightarrow \operatorname{Ell}(B),
$$

then there exists a unital ${ }^{*}$-homomorphism $\varphi: A \rightarrow B$, such that $\varphi$ induces $\alpha$.

Recall that $\operatorname{Ell}(A)=\left(\mathrm{K}_{0}(A), \mathrm{K}_{0}(A)_{+},\left[1_{A}\right]_{0}, \mathrm{~K}_{1}(A), T(A)\right)$. A unital morphism $\alpha: \operatorname{Ell}(A) \rightarrow \operatorname{Ell}(B)$ then consists of a triple $\left(\alpha_{0}, \alpha_{1}, \nu\right)$, where $\alpha_{0}, \alpha_{1}$

\footnotetext{
${ }^{9}$ Here, a dimension drop algebra is an algebra of the form $Z_{p, q}$ with $p, q \in \mathbb{N}$.
} 
are homomorphisms on the respective K-groups, with $\alpha_{0}$ positive and unital, and with $\nu: \operatorname{Aff}(T(A)) \rightarrow \operatorname{Aff}(T(B))$ a unital, positive and affine map. Furthermore, there should be a compatibility between K-theory and traces, in the sense that the following diagram commutes:

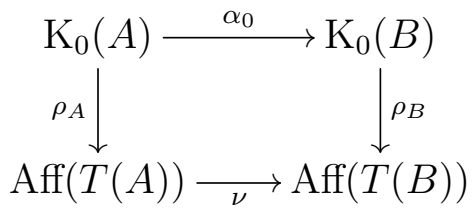

The map $\rho_{A}$ is given by $\rho_{A}\left([p]_{0}\right)(\tau)=(\tau \otimes \operatorname{Tr})(p)$, where $\operatorname{Tr}$ is the standard trace on $\mathbb{K}$, such that $\operatorname{Tr}\left(e_{11}\right)=1$.

The above existence result will be used for the next theorem ([JS99, Proposition 8.5]).

II.2.10 Theorem. There exists a unital ${ }^{*}$-homomorphism $\mathcal{Z} \otimes \mathcal{Z} \rightarrow \mathcal{Z}$.

Proof. We only recall the main steps of the construction of Jiang and $\mathrm{Su}$ (without proof). Let us denote by $A_{n}$ the building blocks of $\mathcal{Z}$, i.e. $A_{n}=$ $Z_{p_{n}, q_{n}}$ as described earlier. Let $B_{n}$ denote the diagonal of $A_{n} \otimes A_{n}$, where we identify $A_{n} \otimes A_{n}$ with continuous functions on $[0,1]^{2}$ satisfying certain boundary conditions. Let us denote the restriction to the diagonal by $\rho_{n}: A_{n} \otimes$ $A_{n} \rightarrow B_{n}$. The goal is then to construct unital ${ }^{*}$-homomorphisms $\varphi_{n}: B_{n} \rightarrow$ $B_{n+1}$, such that the following diagram is a (one-sided) approximate intertwining, where $B:=\underset{\lim }{\longrightarrow}\left(B_{n}, \varphi_{n}\right)$ :

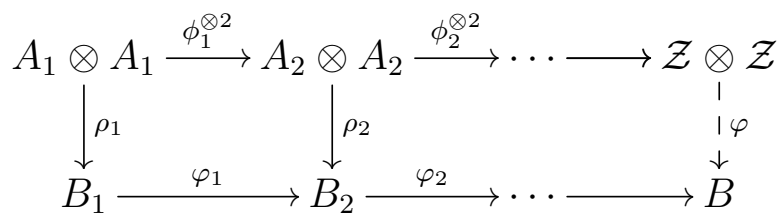

Let us remark that the $\varphi_{n}$ are constructed by hand, without using a uniqueness result. Once this is established, we still need a unital ${ }^{*}$-homomorphism $B \rightarrow \mathcal{Z}$. This is done by invoking Theorem II.2.9. Since each $B_{n}$ is a prime dimension drop algebra, it follows that $\left(\mathrm{K}_{0}(B), \mathrm{K}_{1}(B),\left[1_{B}\right]_{0}\right) \cong(\mathbb{Z}, 0,1)$ and one can show that the limit $B$ is simple and has a unique trace. By writing down a map on the level of the Elliott invariant Ell, we then find a unital ${ }^{*}$-homomorphism $\sigma: B \rightarrow \mathcal{Z}$. Now, the composition $\sigma \circ \varphi: \mathcal{Z} \otimes \mathcal{Z} \rightarrow \mathcal{Z}$ is the required unital ${ }^{*}$-homomorphism. 
We can now use Theorem II.1.7 to show that $\mathcal{Z}$ is strongly self-absorbing. In [JS99, Proposition 8.3] it is proven that $\mathcal{Z}$ has approximately inner half flip. Let us mention that the proof of this fact, different from the purely infinite cases, does not rely on any classification machinery. Indeed, the unitaries implementing the approximate inner half flip come from the unitary paths used in the construction of $\mathcal{Z}$. Then, fix a unital *-homomorphism $\psi: \mathcal{Z} \otimes \mathcal{Z} \rightarrow \mathcal{Z}$. The uniqueness theorem for maps between dimension drop algebras ([JS99, Theorem 7.6, Corollary 5.6]) shows that

$$
\psi \circ\left(\operatorname{id}_{\mathcal{Z}} \otimes 1_{\mathcal{Z}}\right) \approx_{\mathrm{u}} \operatorname{id}_{\mathcal{Z}}
$$

Therefore, all assumptions in Theorem II.1.7 are satisfied and it follows that $\mathcal{Z}$ is strongly self-absorbing.

We see that Jiang and $\mathrm{Su}$ need the full force of classification to show that $\mathcal{Z}$ is strongly self-absorbing. This is because it seems difficult to write down an approximate intertwining between $\mathcal{Z} \otimes \mathcal{Z}$ and $\mathcal{Z}$, only using the construction of $\mathcal{Z}$ by Jiang and $\mathrm{Su}$. In this thesis we will circumvent this problem by using a different picture, due to Rørdam and Winter. 


\section{Chapter III}

\section{Uniqueness}

The aim of this chapter is to prove uniqueness results for standard *-homomorphisms on $Z_{\mathfrak{p}, \mathfrak{q}}$. Using a so-called Basic Homotopy Lemma (Lemma III.4.9, we can prove an asymptotic uniqueness result for standard ${ }^{*}$-homomorphisms $Z_{\mathfrak{p}, \mathfrak{q}} \rightarrow M_{\mathfrak{p}} \otimes M_{\mathfrak{q}}$ and an approximate uniqueness result for standard ${ }^{*}$-endomorphisms $Z_{\mathfrak{p}, \mathfrak{q}} \rightarrow Z_{\mathfrak{p}, \mathfrak{q}}$, see Theorem III.5.4 respectively Theorem III.6.1.

\section{III.1 Classification of Lebesgue contractions}

This section relies on spectral theory and basic properties of UHF-algebras. Even though the main result of this section (Theorem III.1.5) is well-known, I want to provide an elementary proof, as for example described in [Sko16].

III.1.1 Definition. Let $A$ be a unital $\mathrm{C}^{*}$-algebra and $\tau \in T(A)$. We then define the dimension function $d_{\tau}: A_{+} \rightarrow[0, \infty)$ associated to $\tau$ by

$$
d_{\tau}(a):=\lim _{n \rightarrow \infty} \tau\left(a^{\frac{1}{n}}\right) \quad\left(a \in A_{+}\right) .
$$

Note that the limit exists since the sequence $\left(\tau\left(a^{\frac{1}{n}}\right)\right)_{n=1}^{\infty}$ is increasing and bounded by $\|a\|$.

Dimension functions play an important role in the classification of $\mathrm{C}^{*}$ algebras and are closely related to the Cuntz semigroup, as we will see in Chapter V. 
III.1.2 Definition. Let $A$ be a unital $\mathrm{C}^{*}$-algebra and $\tau \in T(A)$. Let $a \in A$ be a self-adjoint element. We then define the eigenvalue function $\lambda_{\tau}^{a}:[0,1) \rightarrow$ $[0, \infty)$ of $a$ with respect to $\tau$ by

$$
\lambda_{\tau}^{a}(s)=\inf \left\{t \in \mathbb{R}: d_{\tau}\left((a-t)_{+}\right) \leq s\right\} .
$$

Here $(a-t)_{+}$denotes the positive part of $a-t 1_{A}$.

The next lemma justifies the name eigenvalue function.

III.1.3 Lemma. Let $A$ be a unital $\mathrm{C}^{*}$-algebra and $\tau \in T(A)$. Let $\left\{p_{i}\right\}_{i=1}^{n}$ be a family of pairwise orthogonal projections in $A$ such that $\sum_{i=1}^{n} p_{i}=1_{A}$. Let $\lambda_{1} \geq \lambda_{2} \geq \cdots \geq \lambda_{n}$ be real numbers and define

$$
a:=\sum_{i=1}^{n} \lambda_{i} p_{i} .
$$

Then, for $k=1,2, \cdots, n$,

$$
\lambda_{\tau}^{a}(s)=\lambda_{k} \quad\left(s \in\left[s_{k-1}, s_{k}\right)\right),
$$

where $s_{k}=\sum_{i=1}^{k} \tau\left(p_{i}\right)$.

Proof. One should think of $a$ as a diagonal operator, with respect to the projections $p_{i}$, having the $\lambda_{i}$ as diagonal entries. For $t \in \mathbb{R}$, we get

$$
(a-t)_{+}=\sum_{i=1}^{n}\left(\lambda_{i}-t\right)_{+} p_{i} .
$$

It is clear that $\left(\lambda_{i}-t\right)_{+}=0$ if $t \geq \lambda_{i}$. It follows that

$$
d_{\tau}\left((a-t)_{+}\right)=\sum_{\left\{i: t<\lambda_{i}\right\}} \tau\left(p_{i}\right)=s_{k^{\prime}}
$$

where $k^{\prime}$ is the largest integer such that $t<\lambda_{k^{\prime}}$. We see that for $s \in\left[s_{k-1}, s_{k}\right)$ we have

$$
d_{\tau}\left((a-t)_{+}\right) \leq s \quad \Longleftrightarrow \quad t \geq \lambda_{k}
$$

Hence $\lambda_{\tau}^{a}(s)=\lambda_{k}$.

III.1.4 Example. Let $A$ be a unital $\mathrm{C}^{*}$-algebra and assume $h \in A_{+}$is a Lebesgue contraction (see Definition I.2.8). Then, the eigenvalue function of 
$a$ with respect to any tracial state $\tau \in T(A)$ can be computed very explicitly (where $\mu$ is the Lebesgue measure):

$$
\begin{aligned}
\lambda_{\tau}^{h}(s) & =\inf \left\{t \in \mathbb{R}: d_{\tau}\left((h-t)_{+}\right) \leq s\right\} \\
& =\inf \left\{t \in \mathbb{R}: \mu\left(\operatorname{supp}\left((\iota-t)_{+}\right)\right) \leq s\right\} \\
& =\inf \{t \in \mathbb{R}: 1-t \leq s\} \\
& =1-s .
\end{aligned}
$$

In particular, any two Lebesgue contractions have the same eigenvalue function.

Using the same idea, the following theorem ([Sko16, Theorem 5.1]) is true in a slightly more general setting. In fact, the proof only requires that the $\mathrm{C}^{*}$ algebra in question has real rank zero and strong comparison of projections w.r.t. a faithful tracial state. Relying on more sophisticated methods, we will also show that $U$ can be replaced by a more complicated $\mathrm{C}^{*}$-algebra, for example the ultrapower $U_{\omega}$ of $U$, cf. Lemma V.2.7.

III.1.5 Theorem. Let $U$ be a UHF-algebra and assume that $h, k \in U$ are two Lebesgue contractions. Then $h \approx_{\mathrm{u}} k$.

Proof. Let $\varepsilon>0$. Since $U$ has real rank zero, there exist positive contractions $h^{\prime}, k^{\prime} \in U$ with finite spectrum such that $\left\|h-h^{\prime}\right\|<\frac{\varepsilon}{4}$ and $\left\|k-k^{\prime}\right\|<$ $\frac{\varepsilon}{4}$. Using an iterative argument ([Sko16, Lemma 4.2]) one can show that there exist pairwise orthogonal families of projections $\left\{p_{i}\right\}_{i=1}^{n},\left\{q_{i}\right\}_{i=1}^{n}$, each of which adds up to the identity, and decreasing scalars $\left\{\alpha_{i}\right\}_{i=1}^{n},\left\{\beta_{i}\right\}_{i=1}^{n}$ such that

$$
\tau_{U}\left(p_{i}\right)=\tau_{U}\left(q_{i}\right) \quad(i=1,2, \cdots, n)
$$

and

$$
h^{\prime}=\sum_{i=1}^{n} \alpha_{i} p_{i}, \quad k^{\prime}=\sum_{i=1}^{n} \beta_{i} q_{i} .
$$

Since $\tau_{U}\left(p_{i}\right)=\tau_{U}\left(q_{i}\right)$ it follows that $p_{i} \sim_{\mathrm{u}} q_{i}$, say $u_{i} p_{i} u_{i}^{*}=q_{i}$. Define

$$
u:=\sum_{i=1}^{n} q_{i} u_{i} p_{i} .
$$

\footnotetext{
${ }^{1}$ The same proof works if we assume that $h$ and $k$ have the same eigenvalue function.
} 
Then $u$ is a unitary in $U$ and $u p_{i} u^{*}=q_{i}$, for all $i$ (see also Lemma III.4.5). From Lemma III.1.3 and (III.1.1, we know that

$$
\alpha_{i}=\lambda_{h^{\prime}}^{\tau_{U}}\left(s_{i}\right), \quad \beta_{i}=\lambda_{k^{\prime}}^{\tau_{U}}\left(s_{i}\right),
$$

for some $s_{i} \in[0,1)$. Furthermore, by [Pet85, Proposition 2 (ii)], it follows that

$$
\left|\lambda_{h^{\prime}}^{\tau_{U}}(s)-\lambda_{h}^{\tau_{U}}(s)\right| \leq\left\|h^{\prime}-h\right\|<\frac{\varepsilon}{4} \quad(s \in[0,1)) .
$$

The same holds for $k^{\prime}$ and $k$, too. Now, we have:

$$
\begin{aligned}
\left\|u h u^{*}-k\right\| & \leq \frac{\varepsilon}{2}+\left\|u h^{\prime} u^{*}-k^{\prime}\right\| \\
& =\frac{\varepsilon}{2}+\left\|\sum_{i=1}^{n}\left(\alpha_{i}-\beta_{i}\right) q_{i}\right\| \\
& =\frac{\varepsilon}{2}+\max _{i}\left|\alpha_{i}-\beta_{i}\right| \\
& =\frac{\varepsilon}{2}+\max _{i}\left\{\left|\lambda_{h^{\prime}}^{\tau_{U}}\left(s_{i}\right)-\lambda_{k^{\prime}}^{\tau_{U}}\left(s_{i}\right)\right|\right. \\
& \leq \varepsilon+\max _{i}\left\{\left|\lambda_{h}^{\tau_{U}}\left(s_{i}\right)-\lambda_{k}^{\tau_{U}}\left(s_{i}\right)\right|\right. \\
& =\varepsilon .
\end{aligned}
$$

In the last line we have an equality, because the eigenvalue function of $h$ and $k$ are equal, as shown in Example III.1.4. It follows that there exists a sequence of unitaries $\left(u_{n}\right)_{n=1}^{\infty}$ in $U$ with $u_{n} h u_{n}^{*} \rightarrow k$, as desired.

\section{III.2 Uniqueness for order zero maps}

The next lemma provides a uniqueness result for c.p.c. order zero maps defined on matrix algebras. Since c.p.c. order zero maps are certain compressions of ${ }^{*}$-homomorphisms, we will use ideas from the classification of ${ }^{*}$-homomorphisms, cf. Example II.2.1.

III.2.1 Lemma. Let $A$ be a unital $\mathrm{C}^{*}$-algebra, $p \in \mathbb{N}$ and assume that $\varphi, \psi: M_{p} \rightarrow A$ are c.p.c. order zero maps, such that $\varphi\left(e_{11}\right) \approx \psi\left(e_{11}\right)$. Then $\varphi \approx \psi$. If in addition $A$ has stable rank one, i.e. the set of invertible elements is dense in $A$, then $\varphi \approx_{\mathrm{u}} \psi$.

Proof. Assume $\varphi\left(e_{11}\right) \approx \psi\left(e_{11}\right)$. By a standard argument, there exists a contraction $v \in A_{\omega}$ with

$$
v \varphi\left(e_{11}\right) v^{*}=\psi\left(e_{11}\right), \quad v^{*} \psi\left(e_{11}\right) v=\varphi\left(e_{11}\right) .
$$


Consider the elements $e_{\varphi}:=\left[\left(h_{\varphi}^{\frac{1}{n}}\right)_{n=1}^{\infty}\right]$ and $e_{\psi}:=\left[\left(h_{\psi}^{\frac{1}{n}}\right)_{n=1}^{\infty}\right]$ in $A_{\omega}$, where $h_{\varphi}$ and $h_{\psi}$ are as in Theorem I.2.3. The elements $e_{\varphi}$ and $e_{\psi}$ act as unit on the image of $\varphi$ respectively $\psi$. Considering $A_{\omega} \subseteq\left(A^{* *}\right)_{\omega}$, we furthermore have that $e_{\varphi} \pi_{\varphi}(x)$ and $e_{\psi} \pi_{\psi}(x)$ are elements in $A_{\omega}$, for every $x \in M_{p}$. One then defines

$$
u:=\sum_{i=1}^{p} \pi_{\psi}\left(e_{i 1}\right) e_{\psi} v e_{\varphi} \pi_{\varphi}\left(e_{1 i}\right) \in A_{\omega}
$$

and computes that

$$
u \varphi\left(e_{i j}\right) u^{*}=\psi\left(e_{i j}\right), \quad u^{*} \psi\left(e_{i j}\right) u=\varphi\left(e_{i j}\right) \quad(i, j=1,2, \cdots, p) .
$$

This shows that $\varphi \approx \psi$. If $A$ has stable rank one, we have a polar decomposition in $A_{\omega}$ (see the proof of Lemma V.2.5). We thus may write $u=v|u|$, for some unitary $v \in A_{\omega}$. This unitary will then implement the unitary equivalence of $\varphi$ and $\psi$ in $A_{\omega}$ (cf. Gab18, Proposition 3.13]). It follows that $\varphi \approx_{\mathrm{u}} \psi$

III.2.2 Remark. Let us recall two standard facts about functional calculus. If $x$ and $y$ are two positive and orthogonal contractions in a $\mathrm{C}^{*}$-algebra, then

$$
f(x+y)=f(x)+f(y) \quad\left(f \in C_{0}(0,1]\right) .
$$

It is enough to check this on polynomials of the form $z \mapsto z^{n}$ :

$$
(x+y)^{n}=\sum_{k=0}^{n}\left(\begin{array}{l}
n \\
k
\end{array}\right) x^{k} y^{n-k}=x^{n}+y^{n} .
$$

Furthermore, if $x$ and $y$ are equivalent, in the sense that $x=a^{*} a$ and $y=a a^{*}$, then

$$
\tau(f(x))=\tau(f(y)) \quad\left(f \in C_{0}(0,1]\right),
$$

where $\tau$ is a tracial state. Again, since $\tau$ is continuous and linear, it is enough to check this on polynomials of the form $z \mapsto z^{n}$. Using that $\tau$ is tracial, we see that

$$
\begin{aligned}
\tau\left(x^{n}\right) & =\tau\left(\left(a^{*} a\right)^{n}\right)=\tau\left(a^{*} a \cdot a^{*} a \cdots a^{*} a\right) \\
& =\tau\left(a a^{*} \cdot a a^{*} \cdots a a^{*}\right)=\tau\left(\left(a a^{*}\right)^{n}\right)=\tau\left(y^{n}\right) .
\end{aligned}
$$

III.2.3 Lemma. Let $\mathfrak{p}$ be a supernatural number and let $\varphi, \psi: M_{\mathfrak{p}} \rightarrow U$ be two c.p.c. order zero maps, where $U$ is a UHF-algebra. Assume that $h_{\varphi}$ and $h_{\psi}$ are both Lebesgue contractions. Then $\varphi \approx_{\mathrm{u}} \psi$. 
Proof. By an easy approximation argument one may assume that $\varphi$ and $\psi$ are defined on $M_{p}$, where $p \in \mathbb{N}$. Note that the positive contractions $\left\{\varphi\left(e_{i i}\right)\right\}_{i=1}^{p}$ are mutually orthogonal and equivalent positive elements, in the sense that there exist $x_{i} \in U$ with $\varphi\left(e_{11}\right)=x_{i}^{*} x_{i}$ and $x_{i} x_{i}^{*}=\varphi\left(e_{i i}\right)$. Using that the $\varphi\left(e_{i i}\right)$ are mutually equivalent, it follows that $\tau_{U}\left(f\left(\varphi\left(e_{11}\right)\right)\right)=\tau_{U}\left(f\left(\varphi\left(e_{i i}\right)\right)\right)$, for $i=1,2, \cdots, p$ and $f \in C_{0}(0,1]$. Using that $h_{\varphi}=\varphi\left(1_{p}\right)$ is a Lebesgue contraction, it follows that

$$
\begin{aligned}
\frac{1}{p} \int_{0}^{1} f(t) d t & =\frac{1}{p} \tau_{U}\left(f\left(\varphi\left(1_{p}\right)\right)\right)=\frac{1}{p} \sum_{i=1}^{p} \tau_{U}\left(f\left(\varphi\left(e_{i i}\right)\right)\right) \\
& =\frac{1}{p} \sum_{i=1}^{p} \tau_{U}\left(f\left(\varphi\left(e_{11}\right)\right)\right)=\tau_{U}\left(f\left(\varphi\left(e_{11}\right)\right)\right)
\end{aligned}
$$

for all $f \in C_{0}(0,1]$. The same computation works for $\psi$ and we see that both $\varphi\left(e_{11}\right)$ and $\psi\left(e_{11}\right)$ have $\frac{1}{p}$ Lebesgue spectral measure. In particular they have the same eigenvalue function, see Definition III.1.2 and [Sko16, Definition 2.6]. It follows by Theorem III.1.5 that $\varphi\left(e_{11}\right) \approx_{\mathrm{u}} \psi\left(e_{11}\right)$ and in particular $\varphi\left(e_{11}\right) \approx \psi\left(e_{11}\right)$. Since $U$ has stable rank one, Lemma III.2.1 shows that $\varphi \approx_{\mathrm{u}} \psi$.

\section{III.3 Uniqueness for maps on dimension drop algebras}

In this section we prove approximate uniqueness results for standard *-homomorphisms $Z_{\mathfrak{p}, \mathfrak{q}} \rightarrow M_{\mathfrak{p}} \otimes M_{\mathfrak{q}}$. This is done by reducing the problem to the commuting cones generating the dimension drop algebra and using the following rotation trick.

III.3.1 Rotation trick. The following provides a method that allows to reduce many arguments about maps on $Z_{\mathfrak{p}, \mathfrak{q}}$ to maps on $C([0,1])$. This ingredient is essential for the proofs of Lemma III.3.2 and Lemma III.4.9.

Let $\varphi: Z_{\mathfrak{p}, \mathfrak{q}} \rightarrow U$ be a unital ${ }^{*}$-homomorphism, where $U$ is some $\mathrm{C}^{*}$ algebra (in the following a UHF-algebra or an ultrapower of such). Let $\mathfrak{p}$ and $\mathfrak{q}$ be supernatural numbers of infinite type. By [DW09, Theorem 2.2], the flip map on $M_{\mathfrak{p}} \otimes M_{\mathfrak{p}}$ and $M_{\mathfrak{q}} \otimes M_{\mathfrak{q}}$ is strongly asymptotically inner, witnessed by say unitary paths $S_{\mathfrak{p}}:[0,1) \rightarrow M_{\mathfrak{p}} \otimes M_{\mathfrak{p}}$ and $S_{\mathfrak{q}}:[0,1) \rightarrow M_{\mathfrak{q}} \otimes M_{\mathfrak{q}}$. For 
$t \in[0,1)$, we define a continuous function

$$
\xi_{t}:[0,1] \rightarrow[0,1): s \mapsto \begin{cases}t & \text { if } s \in[0, t], \\ \text { linear } & \text { if } s \in[t, 1], \\ 0 & \text { if } s=1\end{cases}
$$

and unitary paths

$$
\begin{aligned}
& \dot{W}_{t}:[0,1] \rightarrow M_{\mathfrak{q}} \otimes M_{\mathfrak{q}}: s \mapsto S_{\mathfrak{q}}\left(\xi_{t}(1-s)\right), \\
& \grave{W}_{t}:[0,1] \rightarrow M_{\mathfrak{p}} \otimes M_{\mathfrak{p}}: s \mapsto S_{\mathfrak{p}}\left(\xi_{t}(s)\right) .
\end{aligned}
$$

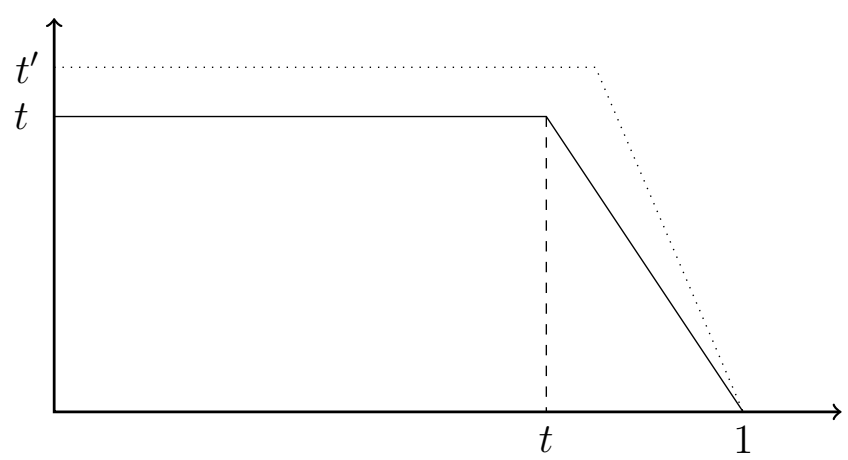

Figure III.1: The function $\xi_{t}$ and $\xi_{t^{\prime}}$ for $t^{\prime}$ slightly larger than $t$.

The path $\hat{W}_{t}$, for example, is designed in such a way that it takes most of the time, namely on $[1-t, 1]$, the value $S_{\mathfrak{q}}(t)$, which implements the flip better and better. Furthermore $W_{t}(0)=1_{\mathfrak{q}} \otimes 1_{\mathfrak{q}}$, showing that $\hat{W}_{t}$ defines an element of the unitization $\left(C_{0}(0,1] \otimes M_{\mathfrak{q}} \otimes M_{\mathfrak{q}}\right)^{+}$and $\hat{W}_{t}-1 \in C_{0}(0,1] \otimes M_{\mathfrak{q}} \otimes M_{\mathfrak{q}}$, where 1 denotes the adjoined unit. Note that we freely identify $C_{0}(0,1] \otimes M_{\mathfrak{q}} \otimes M_{\mathfrak{q}}$ with $\left\{f \in C\left([0,1], M_{\mathfrak{q}} \otimes M_{\mathfrak{q}}\right): f(0)=0\right\}$ and its unitization is then given by $\left\{f \in C\left([0,1], M_{\mathfrak{q}} \otimes M_{\mathfrak{q}}\right): f(0) \in \mathbb{C}\right\}$. Similarly, $\grave{W}_{t} \in\left(C_{0}[0,1) \otimes M_{\mathfrak{p}} \otimes M_{\mathfrak{p}}\right)^{+}$ with scalar part 1 . One then easily checks that

$$
\begin{aligned}
& \lim _{t \rightarrow 1} \dot{W}_{t}(g \otimes r \otimes s)\left(\dot{W}_{t}\right)^{*}=g \otimes s \otimes r, \\
& \lim _{t \rightarrow 1} \grave{W}_{t}(f \otimes x \otimes y)\left(\grave{W}_{t}\right)^{*}=f \otimes y \otimes x,
\end{aligned}
$$

for $g \in C_{0}(0,1], r, s \in M_{\mathfrak{q}}$ and $f \in C_{0}[0,1), x, y \in M_{\mathfrak{p}}$. Since the paths $W_{t}$ and $\grave{W}_{t}$ depend continuously on the parameter $t$, we get unitary paths 
$\left(\grave{U}_{t}^{\varphi}\right)_{t \in[0,1)}$ and $\left(\grave{U}_{t}^{\varphi}\right)_{t \in[0,1)}$ defined by

$$
\begin{aligned}
\grave{U}_{t}^{\varphi} & :=\left(\grave{\varphi} \otimes \mathrm{id}_{M_{\mathfrak{p}}}\right)^{+}\left(\grave{W}_{t}\right) \in U \otimes M_{\mathfrak{p}}, \\
\dot{U}_{t}^{\varphi} & :=\left(\dot{\varphi} \otimes \mathrm{id}_{M_{\mathfrak{q}}}\right)^{+}\left(\dot{W}_{t}\right) \in U \otimes M_{\mathfrak{q}} .
\end{aligned}
$$

See I.3.6 for the definition of $\dot{\varphi}$ and $\grave{\varphi}$. From (III.3.2), it follows that we may twist the non-central part of each cone separately into an extra tensor factor (as done in Lemma III.3.2):

$$
\begin{aligned}
& \lim _{t \rightarrow 1} \dot{U}_{t}^{\varphi}\left(\grave{\varphi}(g \otimes r) \otimes 1_{\mathfrak{q}}\right)\left(\dot{U}_{t}^{\varphi}\right)^{*}=\dot{\varphi}\left(g \otimes 1_{\mathfrak{q}}\right) \otimes r=\varphi(g) \otimes r, \\
& \lim _{t \rightarrow 1} \grave{U}_{t}^{\varphi}\left(\grave{\varphi}(f \otimes x) \otimes 1_{\mathfrak{p}}\right)\left(\grave{U}_{t}^{\varphi}\right)^{*}=\grave{\varphi}\left(f \otimes 1_{\mathfrak{p}}\right) \otimes x=\varphi(f) \otimes x .
\end{aligned}
$$

If we use $U \otimes M_{\mathfrak{p}} \otimes M_{\mathfrak{q}}$ as codomain, as in Lemma III.4.9, we instead define

$$
\begin{aligned}
\grave{U}_{t}^{\varphi} & :=\left(\grave{\varphi} \otimes \imath_{(\mathfrak{p}, \mathfrak{q})}^{[1]}\right)^{+}\left(\grave{W}_{t}\right) \in U \otimes M_{\mathfrak{p}} \otimes M_{\mathfrak{q}}, \\
\dot{U}_{t}^{\varphi} & :=\left(\dot{\varphi} \otimes \imath_{(\mathfrak{p}, \mathfrak{q})}^{[2]}\right)^{+}\left(\dot{W}_{t}\right) \in U \otimes M_{\mathfrak{p}} \otimes M_{\mathfrak{q}},
\end{aligned}
$$

where $\imath_{(\mathfrak{p}, \mathfrak{q})}^{[1]}$ and $\imath_{(\mathfrak{p}, \mathfrak{q})}^{[2]}$ denote the obvious embeddings of $M_{\mathfrak{p}}$ resp. $M_{\mathfrak{q}}$ into the first resp. second tensor factor of $M_{\mathfrak{p}} \otimes M_{\mathfrak{q}}$ (see Notation VI.1.1). In that case

$$
\left[\grave{U}_{t}^{\varphi}, \dot{U}_{t}^{\varphi}\right]=0 \quad(t \in[0,1)) .
$$

Then, the obvious adjustment of (III.3.4) holds:

$$
\begin{array}{ll}
\lim _{t \rightarrow 1} \quad \dot{U}_{t}^{\varphi}\left(\grave{\varphi}(g \otimes r) \otimes 1_{\mathfrak{p q}}\right)\left(\grave{U}_{t}^{\varphi}\right)^{*}=\grave{\varphi}\left(g \otimes 1_{\mathfrak{q}}\right) \otimes 1_{\mathfrak{p}} \otimes r=\varphi(g) \otimes 1_{\mathfrak{p}} \otimes r, \\
\lim _{t \rightarrow 1} \quad \grave{U}_{t}^{\varphi}\left(\grave{\varphi}(f \otimes x) \otimes 1_{\mathfrak{p q}}\right)\left(\grave{U}_{t}^{\varphi}\right)^{*}=\grave{\varphi}\left(f \otimes 1_{\mathfrak{p}}\right) \otimes x \otimes 1_{\mathfrak{q}}=\varphi(f) \otimes x \otimes 1_{\mathfrak{q}},
\end{array}
$$

where $1_{\mathfrak{p q}}:=1_{\mathfrak{p}} \otimes 1_{\mathfrak{q}}$. Most importantly, in this situation we may twist the non-central part of both cones simultaneously into commuting subalgebras of $U \otimes M_{\mathfrak{p}} \otimes M_{\mathfrak{q}}$ :

$$
\begin{aligned}
& \lim _{t \rightarrow 1} \grave{U}_{t}^{\varphi} \grave{U}_{t}^{\varphi}\left(\grave{\varphi}(f \otimes x) \otimes 1_{\mathfrak{p q}}\right)\left(\grave{U}_{t}^{\varphi}\right)^{*}\left(\grave{U}_{t}^{\varphi}\right)^{*}=\varphi(f) \otimes x \otimes 1_{\mathfrak{q}}, \\
& \lim _{t \rightarrow 1} \grave{U}_{t}^{\varphi} \dot{U}_{t}^{\varphi}\left(\grave{\varphi}(g \otimes y) \otimes 1_{\mathfrak{p q}}\right)\left(\dot{U}_{t}^{\varphi}\right)^{*}\left(\grave{U}_{t}^{\varphi}\right)^{*}=\varphi(g) \otimes 1_{\mathfrak{p}} \otimes y,
\end{aligned}
$$

for all $f \in C_{0}[0,1), x \in M_{\mathfrak{p}}$ and $g \in C_{0}(0,1], y \in M_{\mathfrak{q}}$. Note that (III.3.7) follows directly from (III.3.4) and (III.3.6).

III.3.2 Lemma. Let $\mathfrak{p}$ and $\mathfrak{q}$ be supernatural numbers and let $U$ be a UHFalgebra of infinite type, which tensorially absorbs $M_{\mathfrak{p}}$ or $M_{\mathfrak{q}}$. Assume that $\beta_{0}, \beta_{1}: Z_{\mathfrak{p}, \mathfrak{q}} \rightarrow U$ are standard ${ }^{*}$-homomorphisms. Then $\beta_{0} \approx_{\mathrm{u}} \beta_{1}$. 
Proof. Let us assume that $U$ tensorially absorbs $M_{\mathfrak{p}}$. The other case is then obtained by reversing the orientation of the unit interval. By assumption,

$$
h_{\overline{\beta_{0}}}=\dot{\beta}_{0}\left(\iota \otimes 1_{\mathfrak{q}}\right) \quad \text { and } \quad h_{\overline{\beta_{1}}}=\dot{\beta}_{1}\left(\iota \otimes 1_{\mathfrak{q}}\right)
$$

are both Lebesgue contractions and by Lemma III.2.3 we see that $\hat{\beta}_{0} \approx_{\mathrm{u}} \dot{\beta}_{1}$. By Remark I.5.2, there exists a unitary $u \in U_{\omega}$, such that $\operatorname{ad}(u) \circ \dot{\beta}_{0}=\dot{\beta}_{1}$, when $\hat{\beta}_{0}$ and $\hat{\beta}_{1}$ are considered as maps taking values in $U_{\omega}$. Let us define $\gamma_{i}: Z_{\mathfrak{p}, \mathfrak{q}} \rightarrow U_{\omega}$ by

$$
\gamma_{0}:=\operatorname{ad}(u) \circ \beta_{0}, \quad \gamma_{1}:=\beta_{1} .
$$

Then $\dot{\gamma}_{0}=\dot{\gamma}_{1}$ and one easily checks that

$$
\gamma_{0}(f)=\gamma_{1}(f) \quad(f \in C([0,1])) .
$$

For $t \in[0,1)$, we define $V_{t}:=\left(\grave{U}_{t}^{\gamma_{1}}\right)^{*} \grave{U}_{t}^{\gamma_{0}} \in U_{\omega} \otimes M_{\mathfrak{p}} \subseteq\left(U \otimes M_{\mathfrak{p}}\right)_{\omega}$, with $\grave{U}_{t}^{\gamma_{i}}$ as in (III.3.3). Let $f \in C_{0}[0,1), x \in M_{\mathfrak{p}}$ and $\varepsilon>0$. Using (III.3.4), choose $t_{0} \in[0,1)$ such that

$$
\grave{U}_{t}^{\gamma_{i}}\left(\grave{\gamma}_{i}(f \otimes x) \otimes 1_{\mathfrak{p}}\right)\left(\grave{U}_{t}^{\gamma_{i}}\right)^{*} \approx_{\frac{\varepsilon}{2}} \gamma_{i}(f) \otimes x \quad\left(i=0,1, t \geq t_{0}\right) .
$$

For $t \geq t_{0}$, we then get

$$
\begin{aligned}
& V_{t}\left(\grave{\gamma}_{0}(f \otimes x) \otimes 1_{\mathfrak{p}}\right) V_{t}^{*}=\left(\grave{U}_{t}^{\gamma_{1}}\right)^{*} \grave{U}_{t}^{\gamma_{0}}\left(\grave{\gamma}_{0}(f \otimes x) \otimes 1_{\mathfrak{p}}\right)\left(\grave{U}_{t}^{\gamma_{0}}\right)^{*} \grave{U}_{t}^{\gamma_{1}} \\
& \stackrel{\text { III.3.9 }}{\approx} \frac{\varepsilon}{2}\left(\grave{U}_{t}^{\gamma_{1}}\right)^{*}\left(\gamma_{0}(f) \otimes x\right) \grave{U}_{t}^{\gamma_{1}}
\end{aligned}
$$

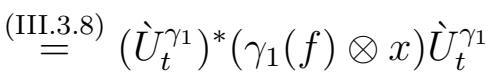

$$
\begin{aligned}
& \stackrel{[I I I .3 .9)}{\approx} \frac{\varepsilon}{2} \grave{\gamma}_{1}(f \otimes x) \otimes 1_{\mathfrak{p}} \text {. }
\end{aligned}
$$

Furthermore, $V_{t}$ commutes with $\dot{\gamma}_{0} \otimes 1_{\mathfrak{p}}=\dot{\gamma}_{1} \otimes 1_{\mathfrak{p}}$. It follows that

$$
\lim _{n \rightarrow \infty}\left\|V_{1-\frac{1}{n}}\left(\gamma_{0}(g) \otimes 1_{\mathfrak{p}}\right) V_{1-\frac{1}{n}}^{*}-\gamma_{1}(g) \otimes 1_{\mathfrak{p}}\right\|=0 \quad\left(g \in Z_{\mathfrak{p}, \mathfrak{q}}\right),
$$

and hence $\gamma_{0} \otimes 1_{\mathfrak{p}} \approx_{\mathrm{u}} \gamma_{1} \otimes 1_{\mathfrak{p}}$. By Remark I.5.2, we have $\gamma_{0} \otimes 1_{\mathfrak{p}} \sim_{\mathrm{u}} \gamma_{1} \otimes 1_{\mathfrak{p}}$. Let $u^{\prime} \in\left(U \otimes M_{\mathfrak{p}}\right)_{\omega}$, such that

$$
\operatorname{ad}\left(u^{\prime}\right) \circ\left(\gamma_{0} \otimes 1_{\mathfrak{p}}\right)=\gamma_{1} \otimes 1_{\mathfrak{p}}
$$

Putting $u^{\prime \prime}:=u^{\prime}\left(u \otimes 1_{\mathfrak{p}}\right) \in\left(U \otimes M_{\mathfrak{p}}\right)_{\omega}$, we thus have $\operatorname{ad}\left(u^{\prime \prime}\right) \circ\left(\beta_{0} \otimes 1_{\mathfrak{p}}\right)=\beta_{1} \otimes 1_{\mathfrak{p}}$. By [TW07, Theorem 2.3] and the assumption that $U \cong U \otimes M_{\mathfrak{p}}$, there exists 
$\mathrm{a}^{*}$-homomorphism $\varphi: U \otimes M_{\mathfrak{p}} \rightarrow U_{\omega}$ with $\varphi\left(x \otimes 1_{\mathfrak{p}}\right)=x$, for $x \in U$. This induces a map

$$
\varphi_{\omega}:\left(U \otimes M_{\mathfrak{p}}\right)_{\omega} \rightarrow\left(U_{\omega}\right)_{\omega} .
$$

Let $w:=\varphi_{\omega}\left(u^{\prime \prime}\right) \in\left(U_{\omega}\right)_{\omega}$. Then

$$
w \beta_{0}(x) w^{*}=\varphi_{\omega}\left(u^{\prime \prime}\right) \varphi_{\omega}\left(\beta_{0}(x) \otimes 1_{\mathfrak{p}}\right) \varphi_{\omega}\left(\left(u^{\prime \prime}\right)^{*}\right)=\varphi_{\omega}\left(\beta_{1}(x) \otimes 1_{\mathfrak{p}}\right)=\beta_{1}(x) .
$$

It follows that $\beta_{0} \sim_{\mathrm{u}} \beta_{1}$ in $\left(U_{\omega}\right)_{\omega}$. By Remark I.5.2 again, $\beta_{0} \approx_{\mathrm{u}} \beta_{1}$ as maps into $U$.

\section{III.4 Basic Homotopy Lemmas}

In this section we prove a so-called Basic Homotopy Lemma for maps $Z_{\mathfrak{p}, \mathfrak{q}} \rightarrow$ $M_{\mathfrak{p}} \otimes M_{\mathfrak{q}}$. Roughly, it says that a unitary in $M_{\mathfrak{p}} \otimes M_{\mathfrak{q}}$, which approximately commutes with the image of such a map, may be connected to the identity by a unitary path, which pointwise approximately commutes with the image very well, too.

Combining this with our approximate uniqueness result (Lemma III.3.2, we arrive at an asymptotic uniqueness result for standard *-homomorphisms $Z_{\mathfrak{p}, \mathfrak{q}} \rightarrow M_{\mathfrak{p}} \otimes M_{\mathfrak{q}}$ and an approximate uniqueness result for standard *endomorphisms $Z_{\mathfrak{p}, \mathfrak{q}} \rightarrow Z_{\mathfrak{p}, \mathfrak{q}}$ (Theorem III.5.3 respectively Theorem III.6.1).

Let us first collect some basic facts about projections and spectral theory, cf. [BEEK98, Section 2].

III.4.1 Lemma. Let $A$ be a $\mathrm{C}^{*}$-algebra and let $p, q$ be projections in $A$ with $\|p-q\|<1$. Then, there exists a partial isometry $v \in A$ with $v v^{*}=p, v^{*} v=$ $q$ and $\|v-q\|<2\|p-q\|$.

III.4.2 Lemma. Let $\varepsilon>0$ and $g \in C([0,1])$. Then, there exists $\delta>0$ such that the following holds: Whenever $h, k$ are positive contractions in some $\mathrm{C}^{*}$-algebra $A$ and $\|h-k\|<\delta$, then $\|g(h)-g(k)\|<\varepsilon$.

III.4.3 Lemma. Let $\varepsilon>0$ and assume that $I, J \subseteq[0,1]$ are two intervals such that $I$ is contained in the interior of $J$. Then, there exists $\delta_{0}>0$ such that the following holds: Whenever $h$ and $k$ are positive contractions with $\|h-k\|<\delta<\delta_{0}$, then

$$
\|p q-q\|<\varepsilon,
$$

where $q=\chi_{I}(k)$ and $p=\chi_{J}(h)$ denote the respective spectral projections. 
The next lemma is maybe less known and contained in [BEEK98, Lemma 2.4].

III.4.4 Lemma. Let $p, q$, e be projections in some unital $\mathrm{C}^{*}$-algebra $A$ with $e \leq p$ and

$$
\|p q-q\| \leq \varepsilon<\frac{1}{6}
$$

Then, there exists a unitary $u$ in $A$ with

$$
e \leq u q u^{*} \leq p
$$

and $\left\|u q u^{*}-q\right\| \leq 10 \varepsilon$.

III.4.5 Lemma. Let $\left(p_{i}\right)_{i=0}^{N}$ and $\left(q_{i}\right)_{i=0}^{N}$ be two families of projections in a unital $\mathrm{C}^{*}$-algebra $A$, each of which sums up to the identity. If for all $i=0,1,2, \cdots, N$, we have $\left\|p_{i}-q_{i}\right\|<\varepsilon \leq 1$, then there exists a unitary $u \in A$ with $u p_{i} u^{*}=q_{i}$, for $i=1,2, \cdots, N$, and $\left\|u-1_{A}\right\| \leq 2(N+1) \varepsilon$.

Proof. Since $\left\|p_{i}-q_{i}\right\|<1$, it follows from Lemma III.4.1 that there exist partial isometries $v_{i} \in A$ such that $v_{i} v_{i}^{*}=p_{i}$ and $v_{i}^{*} v_{i}=q_{i}$. Furthermore, $\left\|v_{i}-p_{i}\right\| \leq 2\left\|q_{i}-p_{i}\right\|<2 \varepsilon$. Note that $v_{i}=p_{i} v_{i}=v_{i} q_{i}=p_{i} v_{i} q_{i}$. Hence $v_{i} v_{j}^{*}=v_{i} q_{i} q_{j} v_{j}^{*}=\delta_{i, j} v_{i} v_{j}^{*}=\delta_{i, j} p_{i}$. Similarly $v_{i}^{*} v_{j}=\delta_{i, j} q_{i}$. Define

$$
u:=\sum_{i=0}^{N} v_{i} .
$$

Then $u$ is a unitary in $A$, since

$$
u u^{*}=\sum_{i, j=0}^{N} v_{i} v_{j}^{*}=\sum_{i, j=0}^{N} \delta_{i, j} p_{i}=\sum_{i=0}^{N} p_{i}=1_{A} .
$$

Similarly, we get $u^{*} u=1_{A}$ and it is clear that $u p_{i} u^{*}=q_{i}$, for all $i$. Finally, we have

$$
\left\|u-1_{A}\right\|=\left\|\sum_{i=0}^{N} v_{i}-p_{i}\right\|<2(N+1) \varepsilon .
$$

The following lemma is a reformulation of [BEEK98, Lemma 5.1], which is therein referred to as Isospectral Homotopy Lemma in Case of a Large Spectral Gap. In modern work this type of lemma is often called a Basic Homotopy Lemma and due to its importance to the classification of $\mathrm{C}^{*}$-algebras 
great effort has been made to generalize the theory of such (cf. [Lin17]). For our purpose however, this elementary result is sufficient.

III.4.6 Lemma. For every $\varepsilon>0$ there exists $\delta>0$ such that the following holds: Whenever $h$ is a positive contraction with full spectrum and $u$ is a unitary in some UHF-algebra $U$ such that

$$
\left\|u h u^{*}-h\right\|<\delta
$$

then there exists a positive contraction $h^{\prime} \in U$ with finite spectrum such that $\left\|h-h^{\prime}\right\|<\varepsilon$ and there exists a unitary path $\left(w_{t}\right)_{t \in[0,1]}$ in $U$ such that $w_{0}=1_{U}$ and

(i) $\left\|w_{t} h^{\prime} w_{t}^{*}-h^{\prime}\right\|<\varepsilon$, for all $t \in[0,1]$,

(ii) $w_{1} h^{\prime} w_{1}^{*}=u h^{\prime} u^{*}$.

Proof. (I) Let $\varepsilon>0$. Choose $N \in \mathbb{N}$ even with $\frac{1}{N}<\frac{\varepsilon}{10}$ and let $\eta:=\frac{\varepsilon}{1000(N+1)}$. Having $N$, we may define intervals

$$
I_{k}:=\left[\frac{N-k+1}{N}, 1\right] \quad\left(k=1, \frac{3}{2}, 2, \frac{5}{2}, \cdots, N+1\right),
$$

so that $\{1\}=I_{1} \subseteq I_{\frac{3}{2}} \subseteq I_{2} \subseteq \cdots \subseteq I_{N+1}=[0,1]$. By Lemma III.4.3, there exists $0<\delta<\frac{\varepsilon}{10}$ such that whenever $h$ and $k$ are positive contractions with $\|h-k\|<\delta$, then for $k=1, \frac{3}{2}, 2, \cdots, N+1$ :

$$
\left\|p_{k} q_{k-\frac{1}{2}}-q_{k-\frac{1}{2}}\right\|<\eta, \quad\left\|q_{k} p_{k-\frac{1}{2}}-p_{k-\frac{1}{2}}\right\|<\eta,
$$

where $p_{k}=\chi_{I_{k}}(h)$ and $q_{k}=\chi_{I_{k}}(k)$.

(II) We now show that $\frac{\delta}{3}$ does the job. To this end, let $h$ be a positive contraction with full spectrum in some UHF-algebra $U$ and let $u \in U$ be a unitary with

$$
\left\|u h u^{*}-h\right\|<\frac{\delta}{3}
$$

Find a positive contraction $h_{0} \in U$ with finite spectrum such that $h_{0}$ has no spectral gaps of length greater or equal to $\frac{1}{2 N}$ and such that $\left\|h-h_{0}\right\|<\frac{\delta}{3}$. Such $h_{0}$ can be found easily since $h$ has full spectrum and $U$ has real rank zero. Then

$$
\left\|u h_{0} u^{*}-h_{0}\right\|<\delta
$$


Let us look at

$$
h^{\prime}:=\frac{1}{N} \sum_{k=1}^{N} p_{k} \in U,
$$

where $p_{k}:=\chi_{I_{k}}\left(h_{0}\right)$. Then, by functional calculus, $\left\|h_{0}-h^{\prime}\right\| \leq \frac{1}{N}$ and hence $\left\|h-h^{\prime}\right\|<\frac{1}{N}+\frac{\delta}{3}<\varepsilon$. Let us define

$$
k^{\prime}:=u h^{\prime} u^{*}=\frac{1}{N} \sum_{k=1}^{N} u p_{k} u^{*}=\frac{1}{N} \sum_{k=1}^{N} q_{k},
$$

where $q_{k}:=u p_{k} u^{*}=\chi_{I_{k}}\left(u h_{0} u^{*}\right)$. Since $\left\|u h_{0} u^{*}-h_{0}\right\|<\delta$, it follows that (III.4.1) holds for the spectral projections $p_{k}, q_{k}$ associated to $h_{0}$ resp. $u h_{0} u^{*}$ and we may apply Lemma III.4.4 to get unitaries $\left(u_{k}\right)_{k=1}^{N}$ and $\left(v_{k}\right)_{k=1}^{N}$ such that

$$
\begin{aligned}
p_{k-1}<p_{k-\frac{1}{2}} & \leq u_{k} q_{k} u_{k}^{*} \leq p_{k+\frac{1}{2}}<p_{k+1} \\
q_{k-1}<q_{k-\frac{1}{2}} & \leq v_{k} p_{k} v_{k}^{*} \leq q_{k+\frac{1}{2}}<q_{k+1}
\end{aligned}
$$

and, with $q_{k}^{\prime}:=u_{k} q_{k} u_{k}^{*}$ resp. $p_{k}^{\prime}:=v_{k} p_{k} v_{k}^{*}$, we have

$$
\left\|q_{k}^{\prime}-q_{k}\right\| \leq 10 \eta, \quad\left\|p_{k}^{\prime}-p_{k}\right\| \leq 10 \eta .
$$

After having done this setup, we are now able to find the desired unitary path $\left(w_{t}\right)_{t \in[0,1]}$. We will construct this path in three steps.

(III) Let us first show how to connect

$$
h^{\prime}=\frac{1}{N}\left(p_{1}+p_{2}+p_{3}+p_{4}+\cdots+p_{N-1}+p_{N}\right)
$$

to

$$
h^{\prime \prime}:=\frac{1}{N}\left(p_{1}+q_{2}^{\prime}+p_{3}+q_{4}^{\prime}+\cdots+p_{N-1}+q_{N}^{\prime}\right) .
$$

Note that III.4.2 entails that for $k=1,2, \cdots, \frac{N}{2}$ :

$$
p_{2 k}-p_{2 k-1} \sim_{\mathrm{u}} q_{2 k}^{\prime}-p_{2 k-1} \text { in }\left(p_{2 k+1}-p_{2 k-1}\right) U\left(p_{2 k+1}-p_{2 k-1}\right) .
$$

Since this corner is again a UHF-algebra it follows that there exist unitary paths $\left(u_{2 k, t}\right)_{t \in\left[0, \frac{1}{3}\right]}$ inside $\left(p_{2 k+1}-p_{2 k-1}\right) U\left(p_{2 k+1}-p_{2 k-1}\right)$ connecting $p_{2 k}-p_{2 k-1}$ and $q_{2 k}^{\prime}-p_{2 k-1}$. One now checks that

$$
w_{t}:=p_{1}+u_{2, t}+u_{4, t}+\cdots+u_{N, t} \quad(t \in[0,1 / 3])
$$


defines a unitary path in $U$ and connects $h^{\prime}$ to $h^{\prime \prime}$, i.e. $w_{0}=1_{U}$ and $w_{\frac{1}{3}} h^{\prime} w_{\frac{1}{3}}^{*}=$ $h^{\prime \prime}$. Furthermore, it is easy to check that $\left\|w_{t} h^{\prime} w_{t}^{*}-h^{\prime}\right\| \leq \frac{2}{N}<\frac{\varepsilon}{5}<\varepsilon$, for $t \in[0,1 / 3]$.

(IV) In the second step we connect $h^{\prime \prime}$ to

$$
k^{\prime \prime}:=\frac{1}{N}\left(p_{1}^{\prime}+q_{2}+p_{3}^{\prime}+q_{4}+\cdots+p_{N-1}^{\prime}+q_{N}\right) .
$$

Since

$$
\begin{aligned}
1_{U} & =p_{1}+\left(q_{2}^{\prime}-p_{1}\right)+\left(p_{3}-q_{2}^{\prime}\right)+\cdots+\left(q_{N}^{\prime}-p_{N-1}\right)+\left(1_{U}-q_{N}^{\prime}\right) \\
& =p_{1}^{\prime}+\left(q_{2}-p_{1}^{\prime}\right)+\left(p_{3}^{\prime}-q_{2}\right)+\cdots+\left(q_{N}-p_{N-1}^{\prime}\right)+\left(1_{U}-q_{N}\right)
\end{aligned}
$$

and, by III.4.4,

$$
\begin{aligned}
& \left\|\left(q_{k}^{\prime}-p_{k-1}\right)-\left(q_{k}-p_{k-1}^{\prime}\right)\right\|<2 \cdot 10 \eta \\
& \left\|\left(p_{k}-q_{k-1}^{\prime}\right)-\left(p_{k}^{\prime}-q_{k-1}\right)\right\|<2 \cdot 10 \eta
\end{aligned}
$$

it follows together with Lemma III.4.5 that there exists a unitary $z \in U$ which conjugates the $q_{2 k}^{\prime}-p_{2 k-1}$ into $q_{2 k}-p_{2 k-1}^{\prime}$ and $p_{2 k+1}-q_{2 k}^{\prime}$ into $p_{2 k+1}^{\prime}-q_{2 k}$, such that

$$
\left\|z-1_{U}\right\| \leq 2(N+1) \cdot 20 \eta=40(N+1) \eta \leq \frac{\varepsilon}{20} .
$$

Clearly $z h^{\prime \prime} z^{*}=k^{\prime \prime}$. Since $-1 \notin \sigma(z)$, we may write $z=e^{i \pi a}$ for some self-adjoint element $a \in U$ with $\|a\|<1$ and one checks that

$$
z_{t}=e^{i 3(t-1 / 3) \pi a} \quad(t \in[1 / 3,2 / 3])
$$

connects $1_{U}$ to $z$ such that $\left\|z_{t}-1_{U}\right\| \leq \frac{\varepsilon}{20}$. Define

$$
w_{t}:=z_{t} w_{\frac{1}{3}} \quad(t \in[1 / 3,2 / 3]) .
$$

For $t \in[1 / 3,2 / 3]$ we then get

$$
\begin{aligned}
\left\|w_{t} h^{\prime} w_{t}^{*}-h^{\prime}\right\| & =\left\|z_{t} h^{\prime \prime} z_{t}^{*}-h^{\prime}\right\| \leq\left\|z_{t} h^{\prime \prime} z_{t}^{*}-h^{\prime \prime}\right\|+\left\|h^{\prime \prime}-h^{\prime}\right\| \\
& \leq 2 \cdot \frac{\varepsilon}{20}+\left\|w_{\frac{1}{3}} h^{\prime} w_{\frac{1}{3}}^{*}-h^{\prime}\right\|<\frac{\varepsilon}{10}+\frac{\varepsilon}{5}<\varepsilon .
\end{aligned}
$$

(V) In the last step we connect $k^{\prime \prime}=w_{\frac{2}{3}} h^{\prime} w_{\frac{2}{3}}^{*}$ to $k^{\prime}=u h^{\prime} u^{*}$ similarly as we connected $h^{\prime}$ to $h^{\prime \prime}$, producing a unitary path $\left(y_{t}\right)_{t \in[2 / 3,1]}$. We finally define

$$
w_{t}:=y_{t} w_{\frac{2}{3}} \quad(t \in[2 / 3,1]) .
$$


Since $\left\|h^{\prime}-k^{\prime}\right\| \leq\left\|h^{\prime}-h_{0}\right\|+\left\|h_{0}-u h_{0} u^{*}\right\|+\left\|u h_{0} u^{*}-u h^{\prime} u^{*}\right\| \leq \frac{1}{N}+\delta+\frac{1}{N}=$ $\frac{2}{N}+\delta$, it follows that for $t \in[2 / 3,1]$, we also have

$$
\begin{aligned}
\left\|w_{t} h^{\prime} w_{t}^{*}-h^{\prime}\right\| & =\left\|y_{t} k^{\prime \prime} y_{t}^{*}-h^{\prime}\right\| \leq\left\|y_{t} k^{\prime \prime} y_{t}^{*}-k^{\prime}\right\|+\left\|k^{\prime}-h^{\prime}\right\| \\
& \leq \frac{2}{N}+\frac{2}{N}+\delta<\frac{2 \varepsilon}{5}+\frac{\varepsilon}{3}<\varepsilon .
\end{aligned}
$$

We are now done, since the path $\left(w_{t}\right)_{t \in[0,1]}$ satisfies $w_{0}=1_{U}, w_{1} h^{\prime} w_{1}^{*}=k^{\prime}=$ $u h^{\prime} u^{*}$ and $\left\|w_{t} h^{\prime} w_{t}^{*}-h^{\prime}\right\|<\varepsilon$, for $t \in[0,1]$.

III.4.7 Corollary. For every $\varepsilon>0$ there exists $\delta>0$ such that the following holds: Whenever $h$ is a positive contraction with full spectrum in a UHFalgebra $U$ and $u \in U$ is a unitary such that

$$
\left\|u h u^{*}-h\right\|<\delta
$$

then there exists a unitary path $\left(u_{t}\right)_{t \in[0,1]}$ in $U$ such that $u_{0}=1_{U}, u_{1}=u$ and

$$
\left\|u_{t} h u_{t}^{*}-h\right\|<\varepsilon \quad(t \in[0,1]) .
$$

Proof. Let $\varepsilon>0$. Choose $0<\delta<\varepsilon$ such that the conclusion of Lemma III.4.6 holds for $\varepsilon$ replaced by $\frac{\varepsilon}{3}$. We show that $\frac{\delta}{3}$ does the job. To this end let $h$ be a positive contraction in some UHF-algebra $U$ and let $u$ be a unitary in $U$ with

$$
\left\|u h u^{*}-h\right\|<\frac{\delta}{3}
$$

By the choice of $\delta$, there exists a positive contraction $h^{\prime} \in U$ with finite spectrum such that $\left\|h-h^{\prime}\right\|<\frac{\varepsilon}{3}$ and there exists a unitary path $\left(z_{t}\right)_{t \in[0,1]}$ in $U$ such that $z_{0}=1_{U}, z_{1} h^{\prime} z_{1}^{*}=u h^{\prime} u^{*}$ and

$$
\left\|z_{t} h^{\prime} z_{t}^{*}-h^{\prime}\right\|<\frac{\varepsilon}{3} \quad(t \in[0,1]) .
$$

Let $w:=z_{1}^{*} u$. Then, the unitary path $\left(v_{t}\right)_{t \in[0,1]}$, given by $v_{t}:=z_{t}^{*} u$, connects $u$ with $w$ and one easily checks that

$$
\left\|v_{t} h v_{t}^{*}-h\right\|<\varepsilon \quad(t \in[0,1]) .
$$

Using that $z_{1} h^{\prime} z_{1}^{*}=u h^{\prime} u^{*}$, it follows that $w$ actually commutes with $h^{\prime}$. Since $h^{\prime}$ has finite spectrum, we can connect $w$ to $1_{U}$ via a unitary path $\left(w_{t}\right)_{t \in[0,1]}$ such that $w_{t}$ commutes with $h^{\prime}$, for every $t \in[0,1]$. Then,

$$
\left\|w_{t} h w_{t}^{*}-h\right\|<\varepsilon \quad(t \in[0,1]) .
$$


The desired path $\left(u_{t}\right)_{t \in[0,1]}$ is now given by

$$
u_{t}= \begin{cases}v_{2 t} & \text { if } t \in[0,1 / 2] \\ w_{2 t-1} & \text { if } t \in[1 / 2,1]\end{cases}
$$

As an easy consequence we get the following Basic Homotopy Lemma for maps on $C([0,1])$ :

III.4.8 Lemma. For every $\varepsilon>0$ and $\mathcal{F} \subseteq C([0,1])$ finite there exists $\delta>0$ such that the following holds: Whenever $\varphi: C([0,1]) \rightarrow U$ is a unital injectiv $£^{2}{ }^{*}$-homomorphism, where $U$ is a UHF-algebra, and $u \in U$ is a unitary such that

$$
\left\|u \varphi(\iota) u^{*}-\varphi(\iota)\right\|<\delta
$$

then there exists a unitary path $\left(u_{t}\right)_{t \in[0,1]}$ in $U$ such that $u_{0}=1_{U}, u_{1}=u$ and

$$
\left\|u_{t} \varphi(f) u_{t}^{*}-\varphi(f)\right\|<\varepsilon \quad(f \in \mathcal{F}, t \in[0,1]) .
$$

Proof. Let $\varepsilon>0$ and $\mathcal{F} \subseteq C([0,1])$ be finite. By Lemma III.4.2 there exists $\eta>0$ such that the following holds: Whenever $h$ and $k$ are positive contractions in a $\mathrm{C}^{*}$-algebra with $\|h-k\|<\eta$, then $\|f(h)-f(k)\|<\varepsilon$, for all $f \in \mathcal{F}$. Now Corollary III.4.7 produces some $\delta>0$ such that the conclusion of the corollary holds for $\varepsilon$ replaced by $\eta$. We claim that this $\delta$ works.

So let $\varphi: C([0,1]) \rightarrow U$ and $u \in U$ be given, where $U$ is some UHFalgebra, such that

$$
\left\|u \varphi(\iota) u^{*}-\varphi(\iota)\right\|<\delta
$$

By the choice of $\delta$ we can connect $u$ to the identity by a unitary path $\left(u_{t}\right)_{t \in[0,1]}$ such that for all $t \in[0,1]$ :

$$
\left\|u_{t} \varphi(\iota) u_{t}^{*}-\varphi(\iota)\right\|<\eta
$$

But then, for $f \in \mathcal{F}$ and $t \in[0,1]$ we get:

$$
\begin{aligned}
\left\|u_{t} \varphi(f) u_{t}^{*}-\varphi(f)\right\| & =\left\|u_{t} f(\varphi(\iota)) u_{t}^{*}-f(\varphi(\iota))\right\| \\
& =\left\|f\left(u_{t} \varphi(\iota) u_{t}^{*}\right)-f(\varphi(\iota))\right\| \\
& <\varepsilon .
\end{aligned}
$$

\footnotetext{
${ }^{2}$ This assumption ensures that $\varphi(\iota)$ has full spectrum, as needed for Lemma III.4.6. However, one can work around this and remove the assumption of full spectrum so that this lemma also works for $\varphi$ not necessarily injective. See [BEEK98.
} 
The last inequality follows from the choice of $\eta$ and (III.4.5).

Using our rotation trick and the previous lemma, we are now able to prove a Basic Homotopy Lemma for maps on dimension drop algebras.

III.4.9 Lemma. For every $\varepsilon>0$ and $\mathcal{F} \subseteq Z_{\mathfrak{p}, \mathfrak{q}}$ finite, where $\mathfrak{p}$ and $\mathfrak{q}$ are supernatural numbers of infinite type, there exists $\delta>0$ and $\mathcal{G} \subseteq Z_{\mathfrak{p}, \mathfrak{q}}$ finite such that the following holds: Whenever $\varphi: Z_{\mathfrak{p}, \mathfrak{q}} \rightarrow U$ is a unital ${ }^{*}{ }_{-}$homomorphism, where $U$ is a UHF-algebra of infinite type that absorbs $M_{\mathfrak{p}}$ and $M_{\mathfrak{q}}$ tensorially, and $u \in U$ is a unitary such that

$$
\left\|u \varphi(g) u^{*}-\varphi(g)\right\|<\delta \quad(g \in \mathcal{G})
$$

then, there exists a unitary path $\left(u_{t}\right)_{t \in[0,1]}$ in $U$ with $u_{0}=1_{U}, u_{1}=u$ and

$$
\left\|u_{t} \varphi(f) u_{t}^{*}-\varphi(f)\right\|<\varepsilon \quad(f \in \mathcal{F}, t \in[0,1]) .
$$

Proof. Let $\varepsilon>0$ and $\mathcal{F} \subseteq Z_{\mathfrak{p}, \mathfrak{q}}$ be finite. We may assume that

$$
\mathcal{F}=\dot{\mathcal{F}} \cup \grave{\mathcal{F}}
$$

where $\dot{\mathcal{F}}=\left\{g_{k} \otimes y_{k}\right\}_{k=1}^{N} \subseteq C_{0}(0,1] \otimes M_{\mathfrak{q}}$ and $\dot{\mathcal{F}}=\left\{f_{k} \otimes x_{k}\right\}_{k=1}^{N} \subseteq C_{0}[0,1) \otimes M_{\mathfrak{p}}$. The two cones are identified with subalgebras of $Z_{\mathfrak{p}, \mathfrak{q}}$ as explained in I.3.6. With (III.3.2), we choose $t_{0} \in[0,1)$ such that for $k=1,2, \cdots, N$ :

$$
\begin{aligned}
& \dot{W}_{t_{0}}\left(g_{k} \otimes y_{k} \otimes 1_{\mathfrak{q}}\right)\left(\dot{W}_{t_{0}}\right)^{*} \approx_{\frac{\varepsilon}{10}} g_{k} \otimes 1_{\mathfrak{q}} \otimes y_{k} \\
& \grave{W}_{t_{0}}\left(f_{k} \otimes x_{k} \otimes 1_{\mathfrak{p}}\right)\left(\grave{W}_{t_{0}}\right)^{*} \approx_{\frac{\varepsilon}{10}} f_{k} \otimes 1_{\mathfrak{p}} \otimes x_{k} .
\end{aligned}
$$

The unitaries $\dot{W}_{t_{0}}$ and $\grave{W}_{t_{0}}$ are defined in (III.3.1). As observed in III.3.1) the scalar part of $\dot{W}_{t_{0}}$ and $\grave{W}_{t_{0}}$ is 1 , so that $\hat{W}_{t_{0}}-1 \in C_{0}(0,1] \otimes M_{\mathfrak{q}} \otimes M_{\mathfrak{q}}$ and $\grave{W}_{t_{0}}-1 \in C_{0}[0,1) \otimes M_{\mathfrak{p}} \otimes M_{\mathfrak{p}}$. We thus may approximate

$$
\begin{aligned}
& \grave{W}_{t_{0}}-1 \approx_{\frac{\varepsilon}{100}} \sum_{i=1}^{K} h_{i} \otimes a_{i} \otimes b_{i}, \\
& \dot{W}_{t_{0}}-1 \approx_{\frac{\varepsilon}{100}} \sum_{i=1}^{K} k_{i} \otimes c_{i} \otimes d_{i},
\end{aligned}
$$

where $h_{i} \in C_{0}[0,1), a_{i}, b_{i} \in M_{\mathfrak{p}}$ and $k_{i} \in C_{0}(0,1], c_{i}, d_{i} \in M_{\mathfrak{q}}$. Furthermore, we clearly may assume that the elements $a_{i}, b_{i}, c_{i}, d_{i}$ are normalized. We then define

$$
\mathcal{G}:=\left\{h_{i} \otimes a_{i}\right\}_{i=1}^{K} \cup\left\{k_{i} \otimes c_{i}\right\}_{i=1}^{K} \cup\{\iota\} \subseteq Z_{\mathfrak{p}, \mathfrak{q}}
$$


where $\iota$ is the canonical generator of $C_{0}(0,1]$. Let $0<\delta<\frac{\varepsilon}{100 K}$ be as in Lemma III.4.8, with $\varepsilon$ replaced by $\frac{\varepsilon}{100}$ and $\mathcal{F}$ replaced by $\left\{f_{k}\right\}_{k=1}^{N} \cup\left\{g_{k}\right\}_{k=1}^{N}$. Let us check that this choice of $\mathcal{G}$ and $\delta$ works.

To this end assume that $\varphi: Z_{\mathfrak{p}, \mathfrak{q}} \rightarrow U$ is a unital ${ }^{*}$-homomorphism, where $U \cong U \otimes M_{\mathfrak{p}} \otimes M_{\mathfrak{q}}$, and $u \in U$ is a unitary such that

$$
\left\|u \varphi(g) u^{*}-\varphi(g)\right\|<\delta \quad(g \in \mathcal{G})
$$

Since $\iota \in \mathcal{G}$, it follows from the choice of $\delta$, that there exists a unitary path $\left(u_{t}\right)_{t \in[0,1]}$ in $U$, such that $u_{0}=1_{U}, u_{1}=u$ and

$$
\left\|u_{t} \varphi(f) u_{t}^{*}-\varphi(f)\right\|<\frac{\varepsilon}{100} \quad\left(f \in\left\{f_{k}\right\}_{k=1}^{N} \cup\left\{g_{k}\right\}_{k=1}^{N}\right) .
$$

Let us define a unitary path

$$
Z_{t}:=\left(\dot{U}_{t_{0}}^{\varphi}\right)^{*}\left(\grave{U}_{t_{0}}^{\varphi}\right)^{*}\left(u_{t} \otimes 1_{\mathfrak{p q}}\right) \dot{U}_{t_{0}}^{\varphi} \grave{U}_{t_{0}}^{\varphi}, \quad(t \in[0,1]),
$$

with $\grave{U}_{t_{0}}^{\varphi}$ and $\dot{U}_{t_{0}}^{\varphi}$ as in III.3.5. We show that $Z_{t}$ commutes sufficiently well with $\varphi(\mathcal{F})$. Let us check this for $\dot{\mathcal{F}}$. The computation for $\dot{\mathcal{F}}$ is analogous. For $t \in[0,1]$ and $k=1,2, \cdots, N$ we get:

$$
\begin{aligned}
& \operatorname{ad}\left(Z_{t}\right) \circ\left(\dot{\varphi}\left(g_{k} \otimes y_{k}\right) \otimes 1_{\mathfrak{p q}}\right) \\
& =\quad\left(\dot{U}_{t_{0}}^{\varphi}\right)^{*}\left(\grave{U}_{t_{0}}^{\varphi}\right)^{*}\left(u_{t} \otimes 1_{\mathfrak{p q}}\right) \dot{U}_{t_{0}}^{\varphi} \grave{U}_{t_{0}}^{\varphi}\left(\dot{\varphi}\left(g_{k} \otimes y_{k}\right) \otimes 1_{\mathfrak{p q}}\right) . \\
& \left(\grave{U}_{t_{0}}^{\varphi}\right)^{*}\left(\hat{U}_{t_{0}}^{\varphi}\right)^{*}\left(u_{t}^{*} \otimes 1_{\mathfrak{p q}}\right) \grave{U}_{t_{0}}^{\varphi} \dot{U}_{t_{0}}^{\varphi}
\end{aligned}
$$

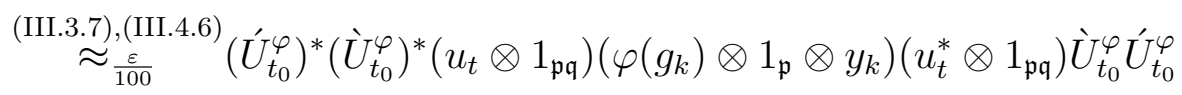

$$
\begin{aligned}
& \underset{\frac{\varepsilon}{100}}{\stackrel{[I I I .49}{\approx}}\left(\dot{U}_{t_{0}}^{\varphi}\right)^{*}\left(\grave{U}_{t_{0}}^{\varphi}\right)^{*}\left(\varphi\left(g_{k}\right) \otimes 1_{\mathfrak{p}} \otimes y_{k}\right) \grave{U}_{t_{0}}^{\varphi} \grave{U}_{t_{0}}^{\varphi} \\
& \stackrel{\text { III.3.7. }}{\underset{\frac{1}{\varepsilon}}{\frac{I I I .4 .6}{100}} \varphi} \varphi\left(g_{k} \otimes y_{k}\right) \otimes 1_{\mathfrak{p q}} \text {. }
\end{aligned}
$$

It follows that

$$
\left\|Z_{t} \varphi(f) Z_{t}^{*}-\varphi(f)\right\|<\frac{\varepsilon}{30} \quad(f \in \mathcal{F}, t \in[0,1]) .
$$

Next, we show that $Z_{1}$ is close to $u \otimes 1_{\mathfrak{p q}}$. To this end, observe that

$$
\begin{aligned}
& \left\|\left(\grave{U}_{t_{0}}^{\varphi}\right)^{*}\left(u \otimes 1_{\mathfrak{p q}}\right) \grave{U}_{t_{0}}^{\varphi}-u \otimes 1_{\mathfrak{p q}}\right\|<\frac{\varepsilon}{30}, \\
& \left\|\left(\dot{U}_{t_{0}}^{\varphi}\right)^{*}\left(u \otimes 1_{\mathfrak{p q}}\right) \dot{U}_{t_{0}}^{\varphi}-u \otimes 1_{\mathfrak{p q}}\right\|<\frac{\varepsilon}{30} .
\end{aligned}
$$


Let us prove that III.4.11 holds for $\grave{U}_{t_{0}}^{\varphi}$. The computation for $\dot{U}_{t_{0}}^{\varphi}$ is similar. Since the image of the scalar part is central it suffices to show that (recall the definition of $\grave{U}_{t_{0}}^{\varphi}$ from (III.3.5) :

$$
\left(u \otimes 1_{\mathfrak{p q}}\right) \cdot\left(\grave{\varphi} \otimes i_{(\mathfrak{p}, \mathfrak{q})}^{[1]}\right)^{+}\left(\grave{W}_{t_{0}}-1\right) \approx_{\frac{\varepsilon}{30}}\left(\grave{\varphi} \otimes i_{(\mathfrak{p}, \mathfrak{q})}^{[1]}\right)+\left(\grave{W}_{t_{0}}-1\right) \cdot\left(u \otimes 1_{\mathfrak{p q}}\right) .
$$

The following calculation shows that this is indeed true:

$$
\begin{aligned}
& \left(u \otimes 1_{\mathfrak{p q}}\right) \cdot\left(\grave{\varphi} \otimes \imath_{(\mathfrak{p}, \mathfrak{q})}^{[1]}\right)^{+}\left(\grave{W}_{t_{0}}-1\right) \\
& \underset{\frac{\varepsilon}{100}}{\stackrel{[I I .4 .7]}{\approx}}\left(u \otimes 1_{\mathfrak{p q}}\right) \cdot\left(\grave{\varphi} \otimes i_{(\mathfrak{p}, \mathfrak{q})}^{[1]}\right)+\left(\sum_{i=1}^{K} h_{i} \otimes a_{i} \otimes b_{i}\right) \\
& =\quad \sum_{i=1}^{K} u \grave{\varphi}\left(h_{i} \otimes a_{i}\right) \otimes b_{i} \otimes 1_{\mathfrak{q}} \\
& \underset{i=1}{\stackrel{\text { III.4.8 }}{\approx} \delta \delta} \sum_{i=1}^{K} \grave{\varphi}\left(h_{i} \otimes a_{i}\right) u \otimes b_{i} \otimes 1_{\mathfrak{q}} \\
& =\quad\left(\grave{\varphi} \otimes \imath_{(\mathfrak{p}, \mathfrak{q})}^{[1]}\right)^{+}\left(\sum_{i=1}^{K} h_{i} \otimes a_{i} \otimes b_{i}\right) \cdot\left(u \otimes 1_{\mathfrak{p q}}\right)
\end{aligned}
$$

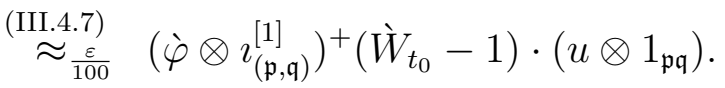

Since $K \delta<\frac{\varepsilon}{100}$, the claim follows. We are now ready to construct the desired unitary path in $U$. By [TW07, Remark 2.7], there exists a unital *-homomorphism $\theta: U \otimes M_{\mathfrak{p}} \otimes M_{\mathfrak{q}} \rightarrow U$ such that

$$
\theta\left(x \otimes 1_{\mathfrak{p q}}\right) \approx_{\frac{\varepsilon}{10}} x \quad(x \in \varphi(\mathcal{F}) \cup\{u\}) .
$$

Let $\left(W_{t}\right)_{t \in[0,1]}$ be given by $W_{t}:=\theta\left(Z_{t}\right)$. Then,

$$
\begin{aligned}
W_{t} \varphi(f) W_{t}^{*} & \approx_{\frac{\varepsilon}{10}} \theta\left(Z_{t}\left(\varphi(f) \otimes 1_{\mathfrak{p q}}\right) Z_{t}^{*}\right) \\
& \approx_{\frac{\varepsilon}{30}} \theta\left(\varphi(f) \otimes 1_{\mathfrak{p q}}\right) \\
& \approx_{\frac{\varepsilon}{10}} \varphi(f),
\end{aligned}
$$

for all $f \in \mathcal{F}$ and $t \in[0,1]$. Next, by (III.4.11], it follows that $\left\|Z_{1}-u \otimes 1_{\mathfrak{p q}}\right\|<$ $\frac{\varepsilon}{15}$, so that $W_{1}=\theta\left(Z_{1}\right) \approx_{\frac{\varepsilon}{15}} \theta\left(u \otimes 1_{\mathfrak{p q}}\right) \approx_{\frac{\varepsilon}{10}} u$. By a standard functional calculus argument, we may extend $\left(W_{t}\right)_{t \in[0,1]}$ to a unitary path $\left(W_{t}\right)_{t \in[0,2]}$ such that $W_{2}=u$ and $\left\|W_{t}-W_{1}\right\|<\frac{\varepsilon}{6}$, for $t \in[1,2]$. Then

$$
\left\|W_{t} \varphi(f) W_{t}^{*}-\varphi(f)\right\|<\varepsilon \quad(t \in[0,2], f \in \mathcal{F}) .
$$

This finishes the proof. 


\section{III.5 Asymptotic uniqueness}

III.5.1 Definition. Assume $A$ is a unital $\mathrm{C}^{*}$-algebra and let $\mathscr{C}$ be a class of unital $\mathrm{C}^{*}$-algebras. We say that $A$ satisfies the Basic Homotopy Property for $\mathscr{C}$ if the following holds:

For every $\varepsilon>0$ and $\mathcal{F} \subseteq A$ finite there exists $\delta>0$ and $\mathcal{G} \subseteq A$ finite such that: If $\varphi: A \rightarrow C \in \mathscr{C}$ is a unital ${ }^{*}$-homomorphism and $u \in C$ is a unitary such that

$$
\left\|u \varphi(g) u^{*}-\varphi(g)\right\|<\delta \quad(g \in \mathcal{G})
$$

then there exists a unitary path $\left(u_{t}\right)_{t \in[0,1]}$ in $C$ such that $u_{0}=1_{C}, u_{1}=u$ and

$$
\left\|u_{t} \varphi(f) u_{t}^{*}-\varphi(f)\right\|<\varepsilon \quad(f \in \mathcal{F}, t \in[0,1]) .
$$

We will abbreviate this and say that $A$ satisfies the (BHP) for $\mathscr{C}$.

III.5.2 Example. It follows from Lemma III.4.8 that $C([0,1])$ satisfies the (BHP) for UHF-algebras. By Lemma III.4.9, any generalized dimension drop algebra $Z_{\mathfrak{p}, \mathfrak{q}}$, with $\mathfrak{p}$ and $\mathfrak{q}$ of infinite type, satisfies the (BHP) for the class

$$
\mathrm{UHF}^{\infty} \otimes M_{\mathfrak{p}} \otimes M_{\mathfrak{q}}
$$

consisting of $M_{\mathfrak{p}} \otimes M_{\mathfrak{q}}$-stable UHF-algebras of infinite type. Furthermore, by [DW09, Lemma 2.1], we have that any strongly self-absorbing $\mathrm{C}^{*}$-algebra $D$ satisfies the (BHP) for

$$
\left\{A \otimes D: A \text { is a unital } \mathrm{C}^{*} \text {-algebra }\right\} .
$$

The next lemma is a standard tool which combines the Basic Homotopy Property and approximate uniqueness to get an asymptotic uniqueness result (cf. [DW09, Theorem 2.2]).

III.5.3 Lemma. Let $A$ be a unital and separable $\mathrm{C}^{*}$-algebra and assume $A$ satisfies the (BHP) for $\mathscr{C}$. Then the following is true: If $\varphi, \psi: A \rightarrow C \in \mathscr{C}$ are unital ${ }^{*}$-homomorphisms and $\varphi \approx_{\mathrm{u}} \psi$, then $\varphi$ and $\psi$ are asymptotically unitarily equivalent. If furthermore the unitary group of $C$ is connected, we get strong asymptotic unitary equivalence, i.e. $\varphi \sim_{\text {asu }} \psi$.

Proof. Let $\varphi, \psi: A \rightarrow C \in \mathscr{C}$ be unital ${ }^{*}$-homomorphisms such that $\varphi \approx_{\mathrm{u}} \psi$. Let $\left(\mathcal{F}_{n}\right)_{n=1}^{\infty}$ be an increasing sequence of finite subsets of $A$ such that their 
union is dense. Let us also fix a decreasing sequence $\varepsilon_{n}>0$ such that $\varepsilon_{n} \rightarrow 0$. Using the Basic Homotopy Property, let $\mathcal{G}_{n} \subseteq A$ be finite and $\delta_{n}>0$ such that the following holds:

Whenever $u \in C$ is a unitary such that

$$
\left\|u^{*} \psi(g) u-\psi(g)\right\|<\delta_{n} \quad\left(g \in \mathcal{G}_{n}\right)
$$

then $u$ is connected to $1_{C}$ via a unitary path that commutes pointwise with $\psi\left(\mathcal{F}_{n}\right)$ up to $\varepsilon_{n}$.

Since $\varphi$ and $\psi$ are are approximately unitarily equivalent, we may choose unitaries $\left(u_{n}\right)_{n=1}^{\infty}$ in $C$, such that

$$
\left\|u_{n}^{*} \varphi(g) u_{n}-\psi(g)\right\|<\frac{\delta_{n}}{2} \quad\left(g \in \mathcal{G}_{n}, n \in \mathbb{N}\right) .
$$

We may furthermore assume that

$$
\mathcal{F}_{n} \subseteq \mathcal{F}_{n+1} \subseteq \mathcal{G}_{n+1} \quad \text { and } \quad \delta_{n+1}<\delta_{n}<\varepsilon_{n} \quad(n \in \mathbb{N}) .
$$

Define

$$
w_{n}:=u_{n+1}^{*} u_{n} \quad(n \in \mathbb{N}) .
$$

One then checks that

$$
\left\|w_{n}^{*} \psi(g) w_{n}-\psi(g)\right\|<\delta_{n} \quad\left(g \in \mathcal{G}_{n}\right) .
$$

By the choice of $\mathcal{G}_{n}$ and $\delta_{n}$, there exists a path $\left(w_{n, t}\right)_{t \in[0,1]}$ of unitaries in $C$ such that $w_{n, 0}=w_{n}, w_{n, 1}=1_{C}$ and

$$
\left\|w_{n, t}^{*} \psi(f) w_{n, t}-\psi(f)\right\|<\varepsilon_{n} \quad\left(f \in \mathcal{F}_{n}, t \in[0,1]\right) .
$$

Let us now define a unitary path $\left(v_{t}\right)_{t \in[0, \infty)}$ in $C$ by

$$
v_{t}=u_{n+1} w_{n, t-n} \quad(t \in[n, n+1)) .
$$

One checks that $v$ is continuous and that

$$
\left\|v_{t} \psi(f) v_{t}^{*}-\varphi(f)\right\|<2 \varepsilon_{n} \quad\left(f \in \mathcal{F}_{n}, t \geq n\right) .
$$

By density, it follows that

$$
\lim _{t \rightarrow \infty}\left\|v_{t} \psi(f) v_{t}^{*}-\varphi(f)\right\|=0 \quad(f \in A) .
$$


By defining

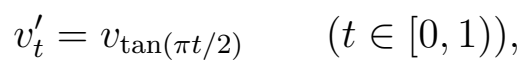

we see that $\varphi$ and $\psi$ are asymptotically unitarily equivalent. Furthermore, if the unitary group of $C$ is connected, we can clearly assume that the unitary path starts at the identity. Hence $\varphi \sim_{\text {asu }} \psi$.

III.5.4 Theorem. Let $\mathfrak{p}$ and $\mathfrak{q}$ be supernatural numbers of infinite type. Assume $\varphi, \psi: Z_{\mathfrak{p}, \mathfrak{q}} \rightarrow U$ are standard ${ }^{*}$-homomorphisms, where $U$ is a UHFalgebra of infinite type that absorbs $M_{\mathfrak{p}}$ and $M_{\mathfrak{q}}$ tensorially. Then $\varphi \sim_{\text {asu }} \psi$.

Proof. We know that $\varphi \approx_{\mathrm{u}} \psi$ by LemmaIII.3.2 and we saw in ExampleIII.5.2 that $Z_{\mathfrak{p}, \mathfrak{q}}$ satisfies the (BHP) for $U$. By Lemma III.5.3 we get $\varphi \sim_{\text {asu }} \psi$.

\section{III.6 Uniqueness for endomorphisms of di- mension drop algebras}

The next theorem shows how the Basic Homotopy Lemma for maps $Z_{\mathfrak{p}, \mathfrak{q}} \rightarrow$ $M_{\mathfrak{p}} \otimes M_{\mathfrak{q}}$ can be lifted to an approximate uniqueness result for standard ${ }^{*}$-endomorphisms of $Z_{\mathfrak{p}, \mathfrak{q}}$.

III.6.1 Theorem. Let $\mathfrak{p}$ and $\mathfrak{q}$ be supernatural numbers of infinite type. Assume that $\varphi, \psi: Z_{\mathfrak{p}, \mathfrak{q}} \rightarrow Z_{\mathfrak{p}, \mathfrak{q}}$ are standard ${ }^{*}$-homomorphisms. Then $\varphi \approx_{\mathrm{u}}$ $\psi$.

Proof. Let $\mathcal{F} \subseteq Z_{\mathfrak{p}, \mathfrak{q}}$ be finite and $\varepsilon>0$. Let $\mathcal{G} \subseteq Z_{\mathfrak{p}, \mathfrak{q}}$ be finite and $0<\delta<\varepsilon$, such that the conclusion of Lemma III.4.9 holds for $\mathcal{F}$ and $\varepsilon$ replaced by $\frac{\varepsilon}{10}$. Let $N \in \mathbb{N}$ such that

$$
|s-t| \leq \frac{1}{N} \quad \Rightarrow \quad\left\|\gamma_{s}(f)-\gamma_{t}(f)\right\|<\frac{\delta}{10} \quad(f \in \mathcal{F} \cup \mathcal{G}, \gamma \in\{\varphi, \psi\}) .
$$

Since $\varphi_{s}, \psi_{s}: Z_{\mathfrak{p}, \mathfrak{q}} \rightarrow M_{\mathfrak{p}} \otimes M_{\mathfrak{q}}$ are standard for each $s \in[0,1]$, we know from Lemma III.3.2 that

$$
\varphi_{\frac{i}{N}} \approx_{\mathrm{u}} \psi_{\frac{i}{N}} \quad(i=0,1,2, \cdots, N-1, N) .
$$

It follows that there exist unitaries $u_{0}, u_{1}, \cdots, u_{N-1}, u_{N} \in M_{\mathfrak{p}} \otimes M_{\mathfrak{q}}$, such that

$$
\left\|u_{i} \varphi_{\frac{i}{N}}(g) u_{i}^{*}-\psi_{\frac{i}{N}}(g)\right\|<\frac{\delta}{5} \quad(g \in \mathcal{G})
$$


We may assume that $u_{0} \in M_{\mathfrak{p}} \otimes 1_{\mathfrak{q}}$ and $u_{N} \in 1_{\mathfrak{p}} \otimes M_{\mathfrak{q}}$. Now, observe that for $i=0,1,2, \cdots, N-1$, we have

$$
\left\|u_{i+1}^{*} u_{i} \varphi_{\frac{i}{N}}(g) u_{i}^{*} u_{i+1}-\varphi_{\frac{i}{N}}(g)\right\|<\delta \quad(g \in \mathcal{G}) .
$$

By the choice of $\mathcal{G}$ and $\delta$, it follows that there exist unitary paths $\left(u_{i, t}\right)_{t \in[0,1]}$ for $i=0,1, \cdots, N-1$, with $u_{i, 0}=1_{\mathfrak{p q}}, u_{i, 1}=u_{i+1}^{*} u_{i}$ and such that:

$$
\left\|u_{i, t} \varphi_{\frac{i}{N}}(f) u_{i, t}^{*}-\varphi_{\frac{i}{N}}(f)\right\|<\frac{\varepsilon}{10} \quad(t \in[0,1], f \in \mathcal{F}) .
$$

This defines a unitary $u$ in $Z_{\mathfrak{p}, \mathfrak{q}}$ by

$$
u_{t}:=u_{i} u_{i, N t-i}^{*} \quad \text { for } \quad t \in\left[\frac{i}{N}, \frac{i+1}{N}\right] .
$$

For $f \in \mathcal{F}$ and $t \in[i / N,(i+1) / N]$, we get

$$
\begin{aligned}
u_{t} \varphi_{t}(f) u_{t}^{*} & \approx_{\frac{\delta}{10}} u_{t} \varphi_{\frac{i}{N}}(f) u_{t}^{*}=u_{i} u_{i, N t-i}^{*} \varphi_{\frac{i}{N}}(f) u_{i, N t-i} u_{i}^{*} \\
& \approx_{\frac{\varepsilon}{10}} u_{i} \varphi_{\frac{i}{N}}(f) u_{i}^{*} \approx_{\frac{\delta}{5}} \psi_{\frac{i}{N}}(f) \\
& \approx_{\frac{\delta}{10}} \psi_{t}(f)
\end{aligned}
$$

As this holds on every interval $[i / N,(i+1) / N]$, it follows that

$$
\left\|u \varphi(f) u^{*}-\psi(f)\right\|<\varepsilon \quad(f \in \mathcal{F}) .
$$

This proves that $\varphi \approx_{\mathrm{u}} \psi$. 


\section{Chapter IV}

\section{Existence}

In this chapter we establish existence results for standard c.p.c. order zero maps and standard ${ }^{*}$-homomorphisms from generalized prime dimension drop algebras into certain UHF-algebras. At the end of this section we construct so-called unitarily suspended ${ }^{*}$-endomorphisms of generalized prime dimension drop algebras. These are particular instances of trace collapsing *-endomorphisms, which will allow us to show that any stationary inductive limit along a fixed generalized prime dimension drop algebra together with such a unitarily suspended *-endomorphism is strongly self-absorbing.

\section{IV.1 Order zero maps}

IV.1.1 Convention. Let $x \in M_{n}$ and $y \in M_{m}$. Whenever we identify $M_{n} \otimes M_{m}$ with $M_{n m}$, we do this via

$$
x \otimes y=\left(x \cdot y_{i j}\right)_{i, j=1}^{m} .
$$

For $p \in \mathbb{N}$, define the UHF-algebra $M_{p^{\infty}}$ to be the limit of

$$
M_{p^{n}} \rightarrow M_{p^{n+1}}: x \mapsto 1_{p} \otimes x .
$$

IV.1.2 Lemma. Let $A$ be a unital $\mathrm{C}^{*}$-algebra. Assume $0 \leq \alpha<\beta \leq 1$. If $h \in A$ is a Lebesgue contraction, then the element

$$
h(\alpha, \beta):=\alpha 1_{A}+(\beta-\alpha) h
$$

has spectrum $[\alpha, \beta]$ and

$$
\tau(f(h(\alpha, \beta)))=\frac{1}{\beta-\alpha} \int_{\alpha}^{\beta} f(t) d t \quad\left(f \in C_{0}(0,1], \tau \in T(A)\right) .
$$


Proof. It is clear that $\sigma(h(\alpha, \beta))=[\alpha, \beta]$, since $\sigma(h)=[0,1]$. For the remaining statement consider the function

$$
g:[0,1] \rightarrow[0,1]: t \mapsto(\beta-\alpha) t+\alpha .
$$

For $f \in C_{0}(0,1]$ we then get by a change of variables:

$$
\tau(f(h(\alpha, \beta)))=\tau((f \circ g)(h))=\int_{0}^{1} f(g(t)) d t=\frac{1}{\beta-\alpha} \int_{\alpha}^{\beta} f(t) d t .
$$

IV.1.3 Remark. (Win09, 1.2.3]) Let $p \geq 2$ be a natural number. For $i, j=2, \cdots, p$ we consider the relations

$$
\left\|x_{i}\right\| \leq 1, x_{i} x_{j}=0(i \neq j), x_{i}^{*} x_{j}=\delta_{i, j} \cdot x_{2}^{*} x_{2} .
$$

Then

$$
C_{0}(0,1] \otimes M_{p} \cong \mathrm{C}^{*}\left(x_{2}, x_{3}, \cdots, x_{p} \mid \mathcal{R}_{p}\right) .
$$

In particular, if $x_{2}, x_{3}, \cdots, x_{p}$ are elements in some $\mathrm{C}^{*}$-algebra $A$ satisfying $\left(\mathcal{R}_{p}\right)$, then the assignment

$$
\varphi\left(e_{i j}\right)=x_{i} x_{j}^{*} \quad(i, j=2,3, \cdots, p)
$$

defines $^{1}$ a c.p.c. order zero map $\varphi: M_{p} \rightarrow A$. Note that

$$
\varphi\left(e_{11}\right)=\varphi\left(e_{i 1}^{*} e_{i 1}\right)=x_{i}^{*} x_{i}=x_{2}^{*} x_{2} \quad(i=2,3, \cdots, p) .
$$

IV.1.4 Lemma. Let $p, q \geq 2$ be natural numbers. Then $M_{q^{\infty}}$ contains elements $x_{2}, x_{3}, \cdots, x_{p}$ satisfying $\left(\mathcal{R}_{p}\right)$ such that $x_{2}^{*} x_{2}$ has $\frac{1}{p}$ Lebesgue spectral measure, i.e. such that

$$
\tau_{M_{q} \infty}\left(f\left(x_{2}^{*} x_{2}\right)\right)=\frac{1}{p} \int_{0}^{1} f(t) d t \quad\left(f \in C_{0}(0,1]\right) .
$$

Proof. Let us recursively define two sequences of non-negative integers $\left(\alpha_{i}\right)_{i=1}^{\infty}$ and $\left(\beta_{i}\right)_{i=0}^{\infty}$. Set $\beta_{0}:=1$ and define $\alpha_{i}$ and $\beta_{i}$ for $i \geq 1$ by

$$
q \beta_{i-1}=p \alpha_{i}+\beta_{i}
$$

${ }^{1}$ More precisely: The elements $\left\{\iota^{\frac{1}{2}} \otimes e_{i 1}: i=2,3, \cdots, p\right\}$ satisfy $\left(\mathcal{R}_{p}\right)$ and hence induce a ${ }^{*}$-homomorphism $C_{0}(0,1] \otimes M_{p} \rightarrow A$, which in turn corresponds to a c.p.c. order zero $\operatorname{map} \varphi: M_{p} \rightarrow A$ satisfying $\varphi\left(e_{i j}\right)=x_{i} x_{j}^{*}$. 
where $\beta_{i} \in\{0,1,2, \cdots, p-1\}$. A short computation using (IV.1.1) shows that the coefficients $\left(\alpha_{i}\right)_{i=1}^{\infty}$ are chosen such that

$$
\sum_{i=1}^{\infty} \frac{p \alpha_{i}}{q^{i}}=1
$$

For further reference, we denote the partial sums by $S_{N}$. We define recursively another sequence $\left(\delta_{i}\right)_{i=0}^{\infty}$ by $\delta_{0}=0$ and for $i \geq 1$ we let

$$
\delta_{i}:=q\left(\delta_{i-1}+p \alpha_{i}\right)
$$

Using IV.1.3 one easily checks that

$$
q^{i+1}=q \beta_{i}+\delta_{i} \quad(i \geq 0)
$$

In particular (IV.1.4 implies that

$$
\delta_{i-1}+p \alpha_{i}+\beta_{i}=q^{i} \quad(i \geq 1) .
$$

All this is made in such a way that we can subdivide the matrix algebra $M_{q^{i}}$ into three orthogonal parts, as indicated in Figure IV.1. Let $h \in M_{q^{\infty}}$ be a

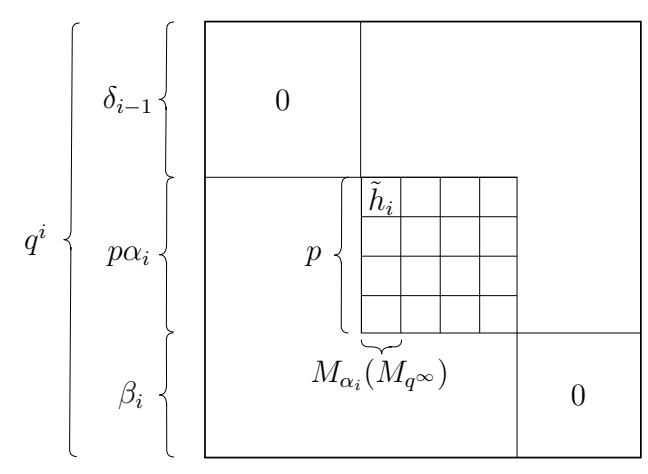

Figure IV.1: Subdivision of $M_{q^{i}}$ and the element $\bar{h}_{i}$.

Lebesgue contraction, for example

$$
h:=\sum_{i=1}^{\infty}\left(\sum_{k=1}^{q-1} \frac{k}{q^{i}} 1_{q}^{\otimes(i-1)} \otimes e_{k k}^{(q)} \otimes 1_{q}^{\otimes \infty}\right) .
$$

For $i \in \mathbb{N}$ and $r \in\left\{1,2, \cdots, \alpha_{i}\right\}$ we set (as in Lemma IV.1.2

$$
\tilde{h}_{i, r}:=h\left(1-S_{i-1}-\frac{r p}{q^{i}}, 1-S_{i-1}-\frac{(r-1) p}{q^{i}}\right) \in M_{q^{\infty}}
$$


and

$$
\tilde{h}_{i}:=\operatorname{diag}\left(\tilde{h}_{i, 1}, \tilde{h}_{i, 2}, \cdots, \tilde{h}_{i, \alpha_{i}}\right) \in M_{\alpha_{i}}\left(M_{q^{\infty}}\right) .
$$

By Lemma IV.1.2, we know

$$
\tau_{M_{q} \infty}\left(f\left(\tilde{h}_{i, r}\right)\right)=\frac{q^{i}}{p} \int_{1-S_{i-1}-\frac{r p}{q^{i}}}^{1-S_{i-1}-\frac{(r-1) p}{q^{i}}} f(t) d t \quad\left(f \in C_{0}(0,1], 1 \leq r \leq \alpha_{i}\right)
$$

and hence

$$
\left(\tau_{M_{q} \infty} \circ \operatorname{Tr}_{\alpha_{i}}\right)\left(f\left(\tilde{h}_{i}\right)\right)=\frac{q^{i}}{p} \int_{1-S_{i}}^{1-S_{i-1}} f(t) d t \quad\left(f \in C_{0}(0,1]\right),
$$

where $\operatorname{Tr}_{\alpha_{i}}$ denotes the sum over the diagonal in $M_{\alpha_{i}}\left(M_{q^{\infty}}\right)$. Next, put $\tilde{h}_{i}$ into the upper left corner of $M_{p}\left(M_{\alpha_{i}}\left(M_{q^{\infty}}\right)\right)$, which we denote by

$$
e_{11}^{(p)} \otimes \tilde{h}_{i}
$$

and set

$$
\bar{h}_{i}:=0_{\delta_{i-1}} \oplus\left(e_{11}^{(p)} \otimes \tilde{h}_{i}\right) \oplus 0_{\beta_{i}} \in M_{q^{i}}\left(M_{q^{\infty}}\right) \cong M_{q^{i}} \otimes M_{q^{\infty}} .
$$

The element $\bar{h}_{i}$ is as depicted in Figure IV.1. Note that by IV.1.5, the element $\bar{h}_{i}$ lives in $M_{q^{i}}\left(M_{q^{\infty}}\right)$. Next, we consider the elements $\bar{h}_{i}$ in

$$
M_{q^{\infty}} \otimes M_{q^{\infty}}=\underline{\lim _{\longrightarrow}}\left(M_{q^{i}} \otimes M_{q^{\infty}}, 1_{q} \otimes \operatorname{id}_{M_{q^{i}}} \otimes \operatorname{id}_{M_{q} \infty}\right) .
$$

By construction, the $\bar{h}_{i}$ are mutually orthogonal positive contractions, which are summable in $M_{q^{\infty}} \otimes M_{q^{\infty}}$. We therefore may define

$$
\bar{h}:=\sum_{i=1}^{\infty} \bar{h}_{i} \in M_{q^{\infty}} \otimes M_{q^{\infty}} .
$$

We can now check that $\bar{h}$ has $\frac{1}{p}$ Lebesgue spectral measure. Denote $T_{N}:=$ $\sum_{i=1}^{N} \bar{h}_{i}$. For $f \in C_{0}(0,1]$ and $N \in \mathbb{N}$ we get:

$$
\begin{gathered}
\tau_{M_{q} \infty \otimes M_{q} \infty}\left(f\left(T_{N}\right)\right)=\sum_{i=1}^{N}\left(\tau_{M_{q^{i}}} \otimes \tau_{M_{q} \infty}\right)\left(f\left(\bar{h}_{i}\right)\right)=\sum_{i=1}^{N} \frac{1}{q^{i}}\left(\tau_{M_{q} \infty} \circ \operatorname{Tr}_{\alpha_{i}}\right)\left(f\left(\tilde{h}_{i}\right)\right) \\
\stackrel{\text { IV.1.6) }}{=} \sum_{i=1}^{N} \frac{1}{q^{i}} \frac{q^{i}}{p} \int_{1-S_{i}}^{1-S_{i-1}} f(t) d t=\frac{1}{p} \int_{1-S_{N}}^{1} f(t) d t .
\end{gathered}
$$


By (IV.1.2) the result follows, if we let $N$ tend to infinity.

In order to finish the proof, instead of $\bar{h}_{i}$, we look, for $j=2,3, \cdots, p$, at the elements

$$
\bar{x}_{j, i}:=0_{\delta_{i-1}} \oplus\left(e_{j 1}^{(p)} \otimes\left(\tilde{h}_{i}\right)^{\frac{1}{2}}\right) \oplus 0_{\beta_{i}} \in M_{q^{i}}\left(M_{q^{\infty}}\right) \cong M_{q^{i}} \otimes M_{q^{\infty}}
$$

and define

$$
x_{j}:=\sum_{i=1}^{\infty} \bar{x}_{j, i} \quad(j=2,3, \cdots, p) .
$$

The elements $\left\{x_{j}\right\}_{j=2}^{p}$ then satisfy $\left(\mathcal{R}_{p}\right)$ and $x_{2}^{*} x_{2}=\bar{h}$, which has $\frac{1}{p}$ Lebesgue spectral measure.

The reader is invited to run this construction for $p=2$ and $q=3$, in which case $\alpha_{i}=\beta_{i}=1$, for all $i \in \mathbb{N}$.

IV.1.5 Lemma. Let $p, q \geq 2$ be natural numbers. Then, there exists a c.p.c. order zero map

$$
\varphi: M_{p} \rightarrow M_{q^{\infty}}
$$

such that $h_{\varphi}$ is a Lebesgue contraction.

Proof. The elements $x_{2}, x_{3}, \cdots, x_{p}$ produced in Lemma IV.1.4 satisfy $\left(\mathcal{R}_{p}\right)$ and hence define, as described in Remark IV.1.3, a c.p.c. order zero map $\varphi: M_{p} \rightarrow M_{q^{\infty}}$ such that $\varphi\left(e_{i j}\right)=x_{i} x_{j}^{*}$, for $i, j=2,3, \cdots, p$. Using that $x_{2}^{*} x_{2}$ has $\frac{1}{p}$ Lebesgue spectral measure, it is now easy to see that $h_{\varphi}$ is a Lebesgue contraction.

\section{IV.2 Unitarily suspended endomorphisms}

IV.2.1 Lemma. Let $p, q \geq 2$ be natural numbers. Then, for $r \in\{p, q\}$, there exist c.p.c. order zero maps $\alpha: M_{p} \rightarrow M_{r \infty}$ and $\beta: M_{q} \rightarrow M_{r \infty}$ such that

$$
\alpha\left(1_{p}\right)+\beta\left(1_{q}\right)=1, \quad\left[\alpha\left(M_{p}\right), \beta\left(M_{q}\right)\right]=\{0\}
$$

and such that $h_{\alpha}, h_{\beta}$ are Lebesgue contractions.

Proof. Let us do the case $r=q$. By LemmaIV.1.5, there exists a c.p.c. order zero map $\phi: M_{p} \rightarrow M_{q \infty}$ such that $h_{\phi}$ is a Lebesgue contraction. We then define c.p.c. order zero maps

$$
\begin{aligned}
& \alpha: M_{p} \rightarrow M_{q^{\infty}} \otimes M_{q}: x \mapsto \phi(x) \otimes 1_{q}, \\
& \beta: M_{q} \rightarrow M_{q^{\infty}} \otimes M_{q}: y \mapsto\left(1-h_{\phi}\right) \otimes y .
\end{aligned}
$$


Since $h_{\phi}$ commutes with the image of $\phi$, we see that the images of $\alpha$ and $\beta$ commute. Furthermore, by definition $\alpha\left(1_{p}\right)+\beta\left(1_{q}\right)=1$ and it is clear that $h_{\alpha}$ and $h_{\beta}$ are Lebesgue contractions.

IV.2.2 Corollary. Let $p, q \geq 2$ be coprime natural numbers. Then, for $r \in\{p, q\}$, there exists a standard ${ }^{*}$-homomorphism

$$
\varphi: Z_{p, q} \rightarrow M_{r \infty}
$$

Proof. Let $r \in\{p, q\}$ and let $\alpha: M_{p} \rightarrow M_{r^{\infty}}$ and $\beta: M_{q} \rightarrow M_{r^{\infty}}$ be as in Lemma IV.2.1. By Theorem I.3.9 these maps induce a standard *-homomorphism $\varphi: Z_{p, q} \rightarrow M_{r \infty}$ such that $\grave{\varphi}=(\bar{\alpha})^{\prime}$ and $\dot{\varphi}=\bar{\beta}$, where $(\cdot)^{\prime}$ means that we have reversed the orientation of the unit interval.

IV.2.3 Convention. Let $\mathfrak{p}$ and $\mathfrak{q}$ be supernatural numbers. We then write $Z_{\mathfrak{p}, \mathfrak{q}}$ as an inductive limit as follows: Fix sequences $\left(P_{n}\right)_{n=1}^{\infty},\left(Q_{n}\right)_{n=1}^{\infty}$ converging (see Section I.3) to $\mathfrak{p}$ respectively $\mathfrak{q}$ such that $P_{n}$ divides $P_{n+1}$ and $Q_{n}$ divides $Q_{n+1}$. This defines unital inclusions $M_{P_{n}} \subseteq M_{P_{n+1}}, M_{Q_{n}} \subseteq M_{Q_{n+1}}$ and $\gamma_{n, n+1}: Z_{P_{n}, Q_{n}} \rightarrow Z_{P_{n+1}, Q_{n+1}}$ such that

$$
Z_{\mathfrak{p}, \mathfrak{q}}=\underset{\lim }{\longrightarrow}\left(Z_{P_{n}, Q_{n}}, \gamma_{n, n+1}\right) .
$$

Let us denote the limit maps by $\gamma_{n}: Z_{P_{n}, Q_{n}} \rightarrow Z_{\mathfrak{p}, \mathfrak{q}}$. Furthermore, we denote by

$$
\kappa_{n}: Z_{\mathfrak{p}, \mathfrak{q}} \rightarrow Z_{P_{n}, Q_{n}}
$$

the induced conditional expectations.

IV.2.4 Lemma. Le $\mathfrak{p}$ and $\mathfrak{q}$ be coprime supernatural numbers. Then, for $\mathfrak{r} \in\{\mathfrak{p}, \mathfrak{q}\}$, there exists a standard ${ }^{*}$-homomorphism

$$
\varphi: Z_{\mathfrak{p}, \mathfrak{q}} \rightarrow M_{\mathfrak{r}}
$$

Proof. Let $\left(\mathcal{F}_{n}\right)_{n=1}^{\infty}$ be an increasing sequence of finite subsets of $Z_{\mathfrak{p}, \mathfrak{q}}$ with dense union, such that $\mathcal{F}_{n} \subseteq Z_{P_{n}, Q_{n}}$ and $\gamma_{n, n+1}\left(\mathcal{F}_{n}\right) \subseteq \mathcal{F}_{n+1}$. Furthermore, let $\varepsilon_{n}>0$ with $\sum \varepsilon_{n}<\infty$. By Corollary IV.2.2, for each $n \in \mathbb{N}$, there exists a standard ${ }^{*}$-homomorphism $\varphi_{n}: Z_{P_{n}, Q_{n}} \rightarrow M_{\mathfrak{r}}$. Note that $\varphi_{n+1} \circ \gamma_{n, n+1}$ is also standard. Hence, by Lemma III.3.2, there exists a unitary $u_{n+1} \in M_{\mathfrak{r}}$ such that

$$
\left\|\varphi_{n}(x)-u_{n+1} \varphi_{n+1}\left(\gamma_{n, n+1}(x)\right) u_{n+1}^{*}\right\|<\varepsilon_{n} \quad\left(x \in \mathcal{F}_{n}\right) .
$$


Define $\tilde{\varphi}_{1}:=\varphi_{1}$ and for $n \geq 2$ we let

$$
\tilde{\varphi}_{n}: Z_{P_{n}, Q_{n}} \rightarrow M_{\mathfrak{r}}: x \mapsto u_{2} u_{3} \cdots u_{n} \varphi_{n}(x) u_{n}^{*} \cdots u_{3}^{*} u_{2}^{*}
$$

One then checks that for each $x \in Z_{P_{n}, Q_{n}}$ the sequence $\left(\tilde{\varphi}_{k}\left(\gamma_{n, k}(x)\right)\right)_{k=n}^{\infty}$ is Cauchy and we may define

$$
\varphi(x):=\lim _{k \rightarrow \infty} \tilde{\varphi}_{k}\left(\gamma_{n, k}(x)\right) \quad\left(x \in Z_{P_{n}, Q_{n}}\right) .
$$

This extends to a ${ }^{*}$-homomorphism $\varphi: Z_{\mathfrak{p}, \mathfrak{q}} \rightarrow M_{\mathfrak{r}}$, which is easily seen to be standard.

IV.2.5 Definition. Let $\mathfrak{p}$ and $\mathfrak{q}$ be supernatural numbers of infinite type. We say that a ${ }^{*}$-homomorphism $\mu: Z_{\mathfrak{p}, \mathfrak{q}} \rightarrow Z_{\mathfrak{p}, \mathfrak{q}}$ is unitarily suspended if

$$
\mu_{t}= \begin{cases}\operatorname{ad}\left(u_{t}\right) \circ\left(\alpha_{0} \otimes 1_{\mathfrak{q}}\right) & \text { if } t \in[0,1), \\ 1_{\mathfrak{p}} \otimes \alpha_{1} & \text { if } t=1\end{cases}
$$

where $\alpha_{0}: Z_{\mathfrak{p}, \mathfrak{q}} \rightarrow M_{\mathfrak{p}}$ and $\alpha_{1}: Z_{\mathfrak{p}, \mathfrak{q}} \rightarrow M_{\mathfrak{q}}$ are unital ${ }^{*}$-homomorphisms and $\left(u_{t}\right)_{t \in[0,1)}$ is a unitary path in $M_{\mathfrak{p}} \otimes M_{\mathfrak{q}}$ starting at the identity.

IV.2.6 Theorem. Let $\mathfrak{p}$ and $\mathfrak{q}$ be coprime supernatural numbers of infinite type. Then, there exists a unitarily suspended standard ${ }^{*}$-homomorphism $\mu: Z_{\mathfrak{p}, \mathfrak{q}} \rightarrow Z_{\mathfrak{p}, \mathfrak{q}}$.

Proof. By Lemma IV.2.4, there exist standard ${ }^{*}$-homomorphisms $\alpha_{0}: Z_{\mathfrak{p}, \mathfrak{q}} \rightarrow$ $M_{\mathfrak{p}}$ and $\alpha_{1}: Z_{\mathfrak{p}, \mathfrak{q}} \rightarrow M_{\mathfrak{q}}$. By Theorem III.5.4, we have $\alpha_{0} \otimes 1_{\mathfrak{q}} \sim_{\text {asu }} 1_{\mathfrak{p}} \otimes \alpha_{1}$, witnessed by a unitary path $\left(u_{t}\right)_{t \in[0,1)}$ starting at the identity. Define a *homomorphism $\mu: Z_{\mathfrak{p}, \mathfrak{q}} \rightarrow Z_{\mathfrak{p}, \mathfrak{q}}$ by $\mu_{t}:=\operatorname{ad}\left(u_{t}\right) \circ\left(\alpha_{0} \otimes 1_{\mathfrak{p}}\right)$ for $t \in[0,1)$ and $\mu_{1}:=1_{\mathfrak{p}} \otimes \alpha_{1}$. Then $\mu$ is standard and unitarily suspended in the sense of Definition IV.2.5. 


\section{Chapter V}

\section{Alternative approaches to classification}

This chapter explains alternative routes leading to classification, as established in Chapter III and Chapter IV]. More precisely, we will reprove Lemma IV.1.5 and Lemma III.2.3, i.e.

(i) there exists a c.p.c. order zero map $\varphi: M_{p} \rightarrow U$ such that $h_{\varphi}$ is a Lebesgue contraction,

(ii) any two c.p.c. order zero maps as in (i) are approximately unitarily equivalent,

where $U$ is a UHF-algebra of infinite type 1 Once these results are established, one proceeds as before to get uniqueness and existence results for maps on generalized dimension drop algebras.

To get (i) and (ii) in a more general and quick fashion, we rely on classification of *-homomorphisms from $C_{0}(0,1]$ into stable rank one $\mathrm{C}^{*}$-algebras by means of the Cuntz semigroup ([CE08]). Furthermore, we provide a proof for (ii) using (i) and the uniqueness of the hyperfinite $\mathrm{II}_{1}$-factor. These von Neumann algebraic methods are inspired by recent methods in classifcation, e.g. SSch18.

In the last section we show how classification by the Cuntz semigroup, as in Rob12], can be used to circumvent all of these methods and how to recover Lemmas IV.2.4 and III.3.2.

\footnotetext{
${ }^{1}$ We also prove (i) and (ii) for more complicated codomains, e.g. ultrapowers of UHFalgebras.
} 
(iii) there exists a standard ${ }^{*}$-homomorphism $Z_{\mathfrak{p}, \mathfrak{q}} \rightarrow U$,

(iv) any two standard ${ }^{*}$-homomorphisms $Z_{\mathfrak{p}, \mathfrak{q}} \rightarrow U$ are approximately unitarily equivalent.

However, note that the results of Section III.4 and in particular the asymptotic uniqueness result (Theorem III.5.4) cannot be proved by the methods used in this chapter.

\section{V.1 The Cuntz semigroup}

Let us briefly recall the Cuntz semigroup of a $\mathrm{C}^{*}$-algebra and the category $\mathbf{C u}$, which has been introduced in [CEI08]. See also [APT11] for an excellent survey and connections to classification.

V.1.1 Definition. Let $A$ be a $\mathrm{C}^{*}$-algebra and $a, b \in A_{+}$. We say that $a$ is Cuntz below $b$ if and only if there exists a sequence $\left(x_{n}\right)_{n=1}^{\infty}$ in $A$ such that

$$
\lim _{n \rightarrow \infty}\left\|x_{n}^{*} b x_{n}-a\right\|=0 .
$$

We denote this relation by $a \preccurlyeq b$. We say that $a$ is Cuntz equivalent to $b$ if and only if $a \preccurlyeq b$ and $b \preccurlyeq a$. This is denoted by $a \sim_{\mathrm{Cu}} b$ and we note that $\sim_{\mathrm{Cu}}$ is an equivalence relation.

V.1.2 The Cuntz semigroup of a $\mathrm{C}^{*}$-algebra. Let $A$ be a $\mathrm{C}^{*}$-algebra. We define

$$
\mathrm{Cu}(A):=(A \otimes \mathbb{K})_{+} / \sim_{\mathrm{Cu}},
$$

where $\mathbb{K}$ denotes the compact operators on $\ell^{2}(\mathbb{N})$. The class of an element $a \in(A \otimes \mathbb{K})_{+}$is denoted by $[a]$. Then $\mathrm{Cu}(A)$ becomes a positively ordered abelian monoid, with [0] as neutral element and where

$$
[a] \leq[b] \quad \stackrel{\text { def }}{\Longleftrightarrow} \quad a \preccurlyeq b \quad\left(a, b \in(A \otimes \mathbb{K})_{+}\right) .
$$

Addition is defined by

$$
[a]+[b]:=[\varphi(a \oplus b)]
$$

where $a \oplus b \in M_{2}(A \otimes \mathbb{K})$ denotes the diagonal sum and $\varphi: M_{2}(A \otimes \mathbb{K}) \rightarrow$ $A \otimes \mathbb{K}$ is any ${ }^{*}$-isomorphism induced by a ${ }^{*}$-isomorphism $\mathbb{K} \otimes M_{2} \rightarrow \mathbb{K}$. This addition does not depend on the choice of isomorphism, since any two such 
isomorphisms are unitarily equivalent. ${ }^{2}$ Then, $\mathrm{Cu}(A)$ is a positively ordered abelian monoid, called the Cuntz semigroup of $A]^{3}$ For a ${ }^{*}$-homomorphism $\phi: A \rightarrow B$ we define

$$
\mathrm{Cu}(\phi): \mathrm{Cu}(A) \rightarrow \mathrm{Cu}(B):[a] \mapsto[\phi(a)],
$$

where $\phi$ is understood to be $\phi \otimes \mathrm{id}_{\mathbb{K}}$. This makes $\mathrm{Cu}$ into a functor from the category of $\mathrm{C}^{*}$-algebras into a certain category $\mathbf{C u}$, which is explained below and was identified in [CEI08.

V.1.3 The category Cu. Let $S$ be a positively ordered abelian monoid. For $x, y \in S$ we say that $x$ is way below $y$ if and only if the following holds: Whenever $\left(y_{n}\right)_{n=1}^{\infty}$ is an increasing sequence in $S$ such that $\sup _{n} y_{n}$ exists and $y \leq \sup _{n} y_{n}$, then there exists some $m \in \mathbb{N}$ such that $x \leq y_{m}$. This will be denoted by $x \ll y$. We say that a sequence $\left(x_{n}\right)_{n=1}^{\infty}$ in $S$ is rapidly increasing if $x_{n} \ll x_{n+1}$, for all $n \in \mathbb{N}$.

The objects of $\mathbf{C u}$ are positively ordered abelian monoids $S$ such that the following four axioms are satisfied:

(O1) Any increasing sequence in $S$ has a supremum in $S$,

(O2) every element in $S$ is the supremum of a rapidly increasing sequence in $S$,

(O3) if $x_{1}, x_{2}, y_{1}, y_{2} \in S$ and $x_{i} \ll y_{i}$, for $i=1,2$, then $x_{1}+x_{2} \ll y_{1}+y_{2}$,

(O4) if $\left(x_{n}\right)_{n=1}^{\infty}$ and $\left(y_{n}\right)_{n=1}^{\infty}$ are increasing sequences in $S$, then

$$
\sup _{n}\left(x_{n}+y_{n}\right)=\sup _{n} x_{n}+\sup _{n} y_{n}
$$

A morphism in $\mathbf{C u}$ is a map $\alpha: S \rightarrow T$, such that

(M1) $\alpha$ is additive and preserves 0 ,

(M2) $\alpha$ is order preserving,

\footnotetext{
${ }^{2}$ By a unitary in $M(A \otimes \mathbb{K})$.

${ }^{3}$ There is also a more classical version called the Cuntz semigroup, usually denoted by $W(A)$, which is given by those equivalence classes in $\mathrm{Cu}(A)$ that are represented by an element in $M_{n}(A)_{+}$, for some $n \in \mathbb{N}$. It is true that $W(A)$ is dense in $\mathrm{Cu}(A)$ and $W(A \otimes \mathbb{K}) \cong \mathrm{Cu}(A)$. See [APT11, Theorem 4.33]
} 
(M3) $\alpha$ preserves suprema of increasing sequences,

(M4) $\alpha$ preserves the way below relation.

If $\alpha$ only satisfies (M1),(M2) and (M3), we say that $\alpha$ is a generalized $\mathbf{C u -}$ morphism.

V.1.4 Example. The Cuntz semigroup of $\mathbb{K}=\mathbb{K}\left(\ell^{2}(\mathbb{N})\right)$ is isomorphic to $\overline{\mathbb{N}}=\mathbb{N} \cup\{\infty\}$. The semigroup structure on $\overline{\mathbb{N}}$ is the obvious one. Furthermore, the isomorphism is given by

$$
\mathrm{Cu}(\mathbb{K}) \rightarrow \overline{\mathbb{N}}:[a] \mapsto \operatorname{rank}(a),
$$

where $\operatorname{rank}(a):=\operatorname{dim}(\operatorname{Im}(a))$.

V.1.5 Definition. Let $S$ be a $\mathbf{C u}$-semigroup and note that $[0, \infty]$ is in a natural way a $\mathbf{C u}$-semigroup. Then, a functional on $S$ is a generalized $\mathbf{C u}-$ morphism $\lambda: S \rightarrow[0, \infty]$, i.e. $\lambda$ is additive and preserves the zero element, order, addition and suprema of increasing sequences. The set of functionals on $S$ will be denoted by $\mathrm{F}(S)$.

The next theorem shows that functionals on $\mathrm{Cu}(A)$ can be described very nicely.

V.1.6 Theorem. There is a bijection

$$
\mathrm{QT}_{2}(A) \rightarrow \mathrm{F}(\mathrm{Cu}(A)): \tau \mapsto d_{\tau}
$$

between the set of lower semicontinuous 2-quasitraces on $A$ and functionals on $\mathrm{Cu}(A)$. The functiona田 $d_{\tau}$ is given by

$$
d_{\tau}([a])=\lim _{n \rightarrow \infty}\left(\tau \otimes \operatorname{Tr}_{\mathbb{K}}\right)\left(a^{\frac{1}{n}}\right) \quad\left(a \in(A \otimes \mathbb{K})_{+}\right) .
$$

If $A$ is exact, then

$$
\mathrm{T}(A) \cong \mathrm{F}(\mathrm{Cu}(A)),
$$

where $\mathrm{T}(A)^{5}$ denotes the non-cancellative cone of lower semicontinuous trace $\AA^{6}$ on $A$. If in addition $A$ is simple, then

$$
\mathrm{T}_{d}(A) \cup\left\{\tau_{\infty}\right\}=\mathrm{T}(A) \cong \mathrm{F}(\mathrm{Cu}(A)),
$$

\footnotetext{
${ }^{4}$ Note that one of the main parts of the proof consists of showing that $d_{\tau}$ is indeed a functional.

${ }^{5}$ Note the subtle difference between the set of tracial states $T(A)$ and the set of lower semicontinuous traces $\mathrm{T}(A)$.

${ }^{6}$ Trace here means a homogeneous map $\tau: A_{+} \rightarrow[0, \infty]$ such that $\tau\left(x^{*} x\right)=\tau\left(x x^{*}\right)$, for all $x \in A$.
} 
where $\mathrm{T}_{d}(A) \subseteq \mathrm{T}(A)$ denotes the set of densely defined lower semicontinuous traces. Moreover, if $A$ is unital we may identify $\mathrm{T}_{d}(A) \cong T(A)$.

Proof. The bijective correspondence is proven in [ERS11, Proposition 4.2]. The statement about exactness follows from [ERS11, Remark 4.5] and essentially relies on a result by Haagerup that 2-quasitraces on exact $\mathrm{C}^{*}$-algebras are traces. For the remaining statement, let us show that if $A$ is simple and $\tau(a)<\infty$, for some $a \in A_{+}$, then $\tau$ is densely defined. We still assume that $\tau$ is a lower semicontinuous trace in the sense of [ERS11, Definition 3.1]. Let $x \in A_{+}$and $\varepsilon>0$. Since $A$ is assumed to be simple, $a$ is full and we know that there exist $x_{1}, \cdots, x_{n} \in A$ with

$$
x \approx_{\varepsilon} \sum_{i=1}^{n} x_{i}^{*} a x_{i} .
$$

Using [APT11, Theorem 2.13], we may assume that

$$
(x-\varepsilon)_{+}=\sum_{i=1}^{n} x_{i}^{*} a x_{i}
$$

Now

$$
\tau\left((x-\varepsilon)_{+}\right)=\sum_{i=1}^{n} \tau\left(a^{\frac{1}{2}}\left(x_{i}^{*} x_{i}\right) a^{\frac{1}{2}}\right) \leq \sum_{i=1}^{n}\left\|x_{i}\right\|^{2} \tau(a)<\infty .
$$

This proves the claim that $\tau$ is densely defined and it immediately follows that

$$
\mathrm{T}(A)=\mathrm{T}_{d}(A) \cup\left\{\tau_{\infty}\right\}
$$

where

$$
\tau_{\infty}(x)= \begin{cases}0 & \text { if } x=0 \\ \infty & \text { otherwise }\end{cases}
$$

If $A$ is unital then $\mathrm{T}_{d}(A) \cong T(A)$, because densely defined traces are finite on $1_{A}$ and hence must be bounded traces. The isomorphism is then given by $\tau \mapsto \frac{\tau}{\|\tau\|}$.

V.1.7 Definition. Let $A$ be a $\mathrm{C}^{*}$-algebra. We then denote

$\mathrm{Cu}(A)_{+}:=\{[a] \in \mathrm{Cu}(A):[a]$ is not represented by a projection in $A \otimes \mathbb{K}\}$. 
V.1.8 Remark. Let $A$ be a $\mathrm{C}^{*}$-algebra of stable rank one. Then $V(A)$, the Murray-von Neumann semigroup of $A$, sits canonically inside $\mathrm{Cu}(A)$ (see [APT11, Lemma 2.20]). Furthermore, by [APT11, Corollary 2.24], $\mathrm{Cu}(A)_{+}$ is an absorbing subsemigroup of $\mathrm{Cu}(A)$ and it follows that

$$
\mathrm{Cu}(A)=V(A) \sqcup \mathrm{Cu}(A)_{+},
$$

where $\sqcup$ means disjoint union. Absorbing means that $V(A)+\mathrm{Cu}(A)_{+} \subseteq$ $\mathrm{Cu}(A)_{+}$.

The next lemma will be used to show that on $\mathrm{Cu}(A)_{+}$certain functionals behave like a $\mathbf{C u}$-morphism.

V.1.9 Lemma. Let $A$ be a $\mathrm{C}^{*}$-algebra and let $x \in \mathrm{Cu}(A)$ and $y \in \mathrm{Cu}(A)_{+}$ such that $x \ll y$. Then, there exists $z \in \mathrm{Cu}(A)$ such that $z \neq 0$ and

$$
x+z \leq y .
$$

Proof. Write $x=[a]$ and $y=[b]$, where $a, b \in(A \otimes \mathbb{K})_{+}$. We may assume that $\|a\|=\|b\|=1$. Since $x \ll y$, there exists $\varepsilon>0$ such that $\left.[a] \leq\left[(b-\varepsilon)_{+}\right]\right]^{7}$ Let $c_{\varepsilon} \in C_{0}(0,1]$ be such that $\left\|c_{\varepsilon}\right\|=1$ and $c_{\varepsilon}(t)=0$ for $t \in[\varepsilon, 1]$. Since 0 is not an isolated point in the spectrum of $b$, it follows that $c_{\varepsilon}(b) \neq 0.8$ Furthermore $c_{\varepsilon}(b)$ is orthogonal to $(b-\varepsilon)_{+}$. We see that

$$
[a]+\left[c_{\varepsilon}(b)\right] \leq\left[(b-\varepsilon)_{+}\right]+\left[c_{\varepsilon}(b)\right]=\left[(b-\varepsilon)_{+}+c_{\varepsilon}(b)\right] \leq[b] .
$$

Let $z:=\left[c_{\varepsilon}(b)\right]$. Then $z \neq 0$ and by the above calculation $x+z \leq y$.

V.1.10 Definition. Let $A$ be a unital $\mathrm{C}^{*}$-algebra. By LAff ${ }_{++}(T(A))$ we denote the semigroup of lower semicontinuous affine real-valued (not necessarily finite) strictly positive functions on $T(A)$. Addition and order is pointwise. Then, there is a map of semigroups

$$
\mathrm{Cu}(A) \rightarrow \operatorname{LAff}_{++}(T(A)):[a] \mapsto \widehat{[a]},
$$

where

$$
\widehat{[a]}(\tau):=d_{\tau}([a]) .
$$

\footnotetext{
${ }^{7}$ This is because $[b]=\sup _{n \in \mathbb{N}}\left[(b-1 / n)_{+}\right]$.

${ }^{8}$ If 0 is isolated in $\sigma(b)$, we easily see that $b$ is represented by a projection, which contradicts the assumption that $y=[b] \in \mathrm{Cu}(A)_{+}$.
} 
Let us define another semigroup (cf. [APT11, Definition 5.4]). The underlying set is

$$
\widetilde{W}(A):=V(A) \sqcup \mathrm{LAff}_{++}(T(A)) .
$$

We extend the addition of $V(A)$ and $\mathrm{LAff}_{++}(T(A))$ by setting

$$
[p]+f:=\widehat{[p]}+f \in \operatorname{LAff}_{++}(T(A)),
$$

where $[p] \in V(A)$ and $f \in \operatorname{LAff}_{++}(T(A))$. The order is extended by

- $f \leq[p]$ if and only if $f(\tau) \leq \tau(p)$, for all $\tau \in T(A)$ and

- $[p] \leq f$ if and only if $\tau(p)<f(\tau)$, for all $\tau \in T(A)$.

V.1.11 Example. Let $M_{\mathfrak{p}}$ be an infinite dimensional UHF-algebra and denote $\mathrm{K}_{0}\left(M_{\mathfrak{p}}\right) \cong \mathbb{Q}(\mathfrak{p}) \subseteq \mathbb{Q} !^{9}$ Then

$$
\widetilde{W}\left(M_{\mathfrak{p}}\right) \cong \mathbb{Q}(\mathfrak{p})_{+} \sqcup(0, \infty],
$$

by identifying $f \in \operatorname{LAff}_{++}\left(T\left(M_{\mathfrak{p}}\right)\right)$ with $f\left(\tau_{M_{\mathfrak{p}}}\right) \in(0, \infty]$ and $[p] \in V\left(M_{\mathfrak{p}}\right)$ with $\left(\tau_{M_{\mathfrak{p}}} \otimes \operatorname{Tr}_{\mathbb{K}}\right)(p)$, where $\tau_{M_{\mathfrak{p}}}$ is the unique trace on $M_{\mathfrak{p}}$. In order to distinguish elements in $\mathbb{Q}(\mathfrak{p})_{+}$and $(0, \infty]$, it is convenient to denote elements in $(0, \infty) \subseteq \widetilde{W}\left(M_{\mathfrak{p}}\right)$ by $\alpha^{\prime}$, where $\alpha \in(0, \infty)$. Then the order is given by the usual order on the two distinguished subsets and for $\alpha \in(0, \infty)$ and $\beta \in \mathbb{Q}(\mathfrak{p})_{+}$, we have

- $\alpha^{\prime} \leq \beta$ if and only if $\alpha \leq_{\mathbb{R}} \beta$ and

- $\beta \leq \alpha^{\prime}$ if and only if $\beta<_{\mathbb{R}} \alpha$.

The next lemma gives a more explicit description of the Cuntz semigroup of certain $\mathrm{C}^{*}$-algebras, cf. [APT11, Proposition 5.26].

V.1.12 Lemma. Let $A$ be a simple, unital, exact $\mathrm{C}^{*}$-algebra with stable rank one and strict comparison (see Definition V.2.1 for strict comparison). Then, the map ${ }^{10}$

$$
\Gamma: \mathrm{Cu}(A) \rightarrow V(A) \sqcup \mathrm{LAff}_{++}(T(A))(=\widetilde{W}(A))
$$

\footnotetext{
${ }^{9}$ Recall that $\mathbb{Q}(\mathfrak{p})=\left\{\frac{k}{\prod_{p \in \mathcal{F}} p^{\kappa_{p}}}: k \in \mathbb{Z}, \mathcal{F} \subseteq \mathbb{P}\right.$ finite, $\left.\kappa_{p}<\infty, \kappa_{p} \leq \nu_{p}(\mathfrak{p})\right\}$, where $\mathbb{P}$ denotes the set of prime numbers.

${ }^{10}$ To define $\Gamma$ we use that $\mathrm{Cu}(A)=V(A) \sqcup \mathrm{Cu}(A)_{+}$.
} 
given by

$$
\Gamma([a])= \begin{cases}{[a]} & \text { if }[a] \in V(A), \\ \widehat{[a]} & \text { if }[a] \in \mathrm{Cu}(A)_{+}\end{cases}
$$

is an order embedding.

V.1.13 Corollary. Let $U$ be a UHF-algebra of infinite type. Then

$$
\mathrm{Cu}(U) \cong V(U) \sqcup(0, \infty]=\widetilde{W}(U) .
$$

The isomorphism is the map $\Gamma$ from Lemma $V .1 .12$.

Proof. It is a standard result that UHF-algebras have strict comparison, cf. Rør92. By Lemma V.1.12, we know that $\Gamma$ is an order embedding, so it suffices to show that $\Gamma$ is surjective. As before, we identify $\operatorname{LAff}_{++}(T(U))$ with $(0, \infty)$, by identifying $f$ with $f\left(\tau_{U}\right)$, where $\tau_{U}$ is the unique trace on $U$. To show that $\Gamma$ is surjective, it remains to show that the map

$$
\kappa: \mathrm{Cu}(U)_{+} \rightarrow(0, \infty]:[x] \mapsto d_{\tau_{U}}([x])
$$

is surjective. To see this let $h \in U$ be a Lebesgue contraction ${ }^{11}$ and $\alpha \in(0,1]$. Let $f \in C_{0}(0,1]_{+}$be such that $\|f\|=1$ and $\mu(\operatorname{supp}(f))=\alpha$, where $\mu$ is the Lebesgue measure on $(0,1]$. Then

$$
d_{\tau_{U}}(f(h))=\lim _{n \rightarrow \infty} \tau_{U}\left(f(h)^{\frac{1}{n}}\right)=\lim _{n \rightarrow \infty} \int_{0}^{1} f^{\frac{1}{n}}(t) d t=\mu(\operatorname{supp}(f))=\alpha .
$$

Note that 0 is not an isolated point in $\sigma(f(h))$, because $\sigma(f(h))=f(\sigma(h))=$ $f([0,1])=[0,1]$. By [APT11, Proposition 2.23] we have $[f(h)] \in \mathrm{Cu}(U)_{+}$and it follows that $\alpha \in \operatorname{Im}(\kappa)$. If now $\beta \in(0, \infty)$, let $n \in \mathbb{N}$ with $\frac{\beta}{n} \in(0,1]$. Let $x \in \mathrm{Cu}(U)_{+}$with $\kappa(x)=\frac{\beta}{n}$. Then $n x \in \mathrm{Cu}(U)_{+}$and $\kappa(n x)=n \kappa(x)=\beta$. The element $\infty \in \widetilde{W}(U)$ is given by $\kappa\left(\left[1_{U} \otimes k\right]\right)$, where $k$ is the compact diagonal operator with diagonal $\left(\frac{1}{n}\right)_{n=1}^{\infty}$. This finishes the proof.

The next theorem is due to Ciuperca and Elliott ([CE08]) and is proven to hold for a more general class of target algebras in [RS10, Theorem 2].

V.1.14 Theorem. Assume $A$ is a $\mathrm{C}^{*}$-algebra that has stable rank one. Then, for any $\mathbf{C u - m o r p h i s m ~}$

$$
\alpha: \mathrm{Cu}\left(C_{0}(0,1]\right) \rightarrow \mathrm{Cu}(A),
$$

\footnotetext{
${ }^{11}$ See the proof of Lemma $\overline{I V .1 .4}$ for an explicit formula for such a Lebesgue contraction.
} 
such that $\alpha([\iota]) \leq\left[h_{A}\right]$, where $h_{A} \in A$ is strictly positive, there exists a ${ }^{*}$ homomorphism $\varphi: C_{0}(0,1] \rightarrow A$ such that $\mathrm{Cu}(\varphi)=\alpha$. Furthermore, $\alpha$ is unique up to approximate unitary equivalence by unitaries in $(A \otimes \mathbb{K})^{+}{ }^{12}$

Building on this Robert proved the following ${ }^{13}$ in [Rob12]:

V.1.15 Theorem. Let $A$ be a unital one dimensional non-commutative $\mathrm{CW}$ complex with trivial $\mathrm{K}_{1}$-group or a unital sequential inductive limit of such. Let $B$ be a unital stable rank one $\mathrm{C}^{*}$-algebra. Then, for every $\mathbf{C u}$-morphism

$$
\alpha: \mathrm{Cu}(A) \rightarrow \mathrm{Cu}(B)
$$

such that $\alpha\left(\left[1_{A}\right]\right)=\left[1_{B}\right]$, there exists a unital ${ }^{*}$-homomorphism $\varphi: A \rightarrow B$ such that $\mathrm{Cu}(\varphi)=\alpha$. Furthermore, $\varphi$ is unique up to approximate unitary equivalence.

V.1.16 Remark. By definition a one dimensional non-commutative CW complex $A$ is given by a pullback

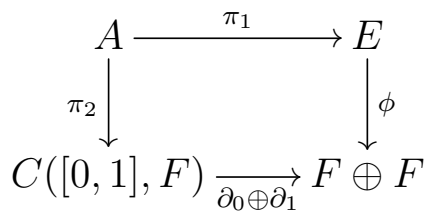

where $E$ and $F$ are finite dimensional $\mathrm{C}^{*}$-algebras and $\phi: E \rightarrow F \oplus F$ is a *-homomorphism. The maps $\partial_{i}$ are the evaluations at the endpoints. We already saw in Lemma I.3.3 that $Z_{p, q}$ is such a pullback, for $p, q \in \mathbb{N}$. Hence Theorem V.1.15 also applies to generalized dimension drop algebras.

\section{V.2 Uniqueness for order zero maps}

V.2.1 Definition. Let $A$ be a unital $\mathrm{C}^{*}$-algebra. We say that $A$ has strict comparison of positive elements by bounded traces if for all $a, b \in M_{n}(A)_{+}$, we have

$$
d_{\tau}(a)<d_{\tau}(b) \quad(\tau \in T(A))
$$

\footnotetext{
${ }^{12}$ If $A$ is unital, we may assume that the unitaries implementing the approximate unitary equivalence live in $A$.

${ }^{13}$ He actually proved a more general result for possibly non-unital $\mathrm{C}^{*}$-algebras. However, we will not need this version and hence omit it.
} 
implies that $a \preccurlyeq b$ in $M_{n}(A)$. Recall that the functional $d_{\tau}$ is given by

$$
d_{\tau}(x)=\lim _{n \rightarrow \infty}\left(\tau \otimes \operatorname{Tr}_{n}\right)\left(x^{\frac{1}{n}}\right) \quad\left(x \in M_{n}(A)_{+}\right),
$$

where $\operatorname{Tr}_{n}$ is the sum over the diagonal on $M_{n}$.

The following is [TWW17, Lemma 2.1 (ii)].

V.2.2 Lemma. Let $A$ be a unital $\mathrm{C}^{*}$-algebra that has strict comparison of positive elements by bounded traces. If $h, k \in A$ are two Lebesgue contractions, then

$$
\mathrm{Cu}\left(\phi_{h}\right)=\mathrm{Cu}\left(\phi_{k}\right),
$$

where

$$
\phi_{h}, \phi_{k}: C_{0}(0,1] \rightarrow A
$$

are the ${ }^{*}$-homomorphisms induced by the given contractions.

Proof. It suffices to show that $f(h) \sim_{\mathrm{Cu}} f(k)$, for every non-zero $f \in C_{0}(0,1]_{+}$. Let $\varepsilon>0$. Then, there exists a small open interval, say $I=(a, b) \cap(0,1]$ such that $f(t) \in(0, \varepsilon)$, for $t \in I$. In particular $(f-\varepsilon)_{+}$vanishes on $I$. Let $c_{I} \in C_{0}(0,1]_{+}$be of norm one such that $c_{I}(t)=0$ for $t \notin I$, see Figure V.1. Then

$$
d_{\tau}\left(c_{I}(h)\right)>0 \quad(\tau \in T(A))
$$

Furthermore, by the choice of $I$ we have that

$$
(f-\varepsilon)_{+}+c_{I} \preccurlyeq f .
$$

This is because in commutative $\mathrm{C}^{*}$-algebras, Cuntz subequivalence is determined by the support of functions (cf. [APT11, Proposition 2.5]). Using that

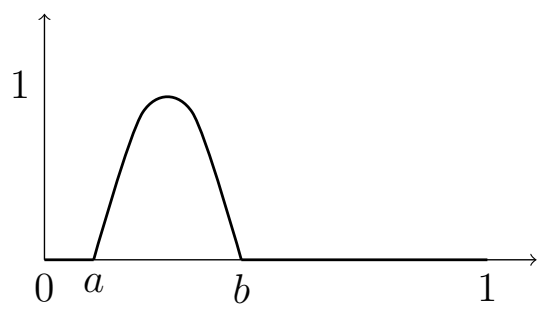

Figure V.1: The function $c_{I}$. 
$c_{I}(h)$ and $(f(h)-\varepsilon)_{+}$are orthogonal, we get for every $\tau \in T(A)$ :

$$
\begin{aligned}
d_{\tau}\left((f(h)-\varepsilon)_{+}\right) & \stackrel{\text { V.2.1 }}{<} d_{\tau}\left((f(h)-\varepsilon)_{+}\right)+d_{\tau}\left(c_{I}(h)\right) \\
& =d_{\tau}\left((f(h)-\varepsilon)_{+}+c_{I}(h)\right) \\
& \stackrel{\mathrm{V} .2 .2}{\leq} d_{\tau}(f(h)) \\
& =d_{\tau}(f(k)) .
\end{aligned}
$$

The last equality follows, because $h$ and $k$ are both Lebesgue contractions. Now, by strict comparison, it follows that

$$
(f(h)-\varepsilon)_{+} \preccurlyeq f(k)
$$

and hence

$$
[f(h)]=\sup _{n}\left[\left(f(h)-\frac{1}{n}\right)_{+}\right] \leq[f(k)] .
$$

Reversing the roles of $h$ and $k$, we see that $[f(k)] \leq[f(h)]$ and thus $f(h) \sim_{\mathrm{Cu}}$ $f(k)$.

V.2.3 Definition. Let us denote by $\mathscr{A}$ the class of unital $\mathrm{C}^{*}$-algebras that have stable rank one and strict comparison of positive elements by bounded traces.

V.2.4 Example. The easiest and for us most interesting class of $\mathrm{C}^{*}$-algebras that belong to $\mathscr{A}$ is the class of UHF-algebras. The stable rank one property is easy to check. Strict comparison is proven in $[\mathrm{R} \varnothing \mathrm{r} 92]$ and relies on the fact that matrix algebras enjoy this property.

V.2.5 Lemma. Let $A \in \mathscr{A}$ and assume $T\left(A_{\omega}\right)=T(A)_{\omega}$, where we recall that $T(A)_{\omega}$ denotes the set of limit traces, i.e. those of the form

$$
\tau_{\omega}\left(\left[\left(x_{n}\right)_{n=1}^{\infty}\right]\right)=\lim _{\omega} \tau\left(x_{n}\right) \quad(\tau \in T(A)) .
$$

Then $A_{\omega} \in \mathscr{A}$.

Proof. Using that $T\left(A_{\omega}\right)=T(A)_{\omega}$ and $\left[\mathrm{BBS}^{+} 19\right.$, Lemma 1.23], it follows that $A_{\omega}$ has strict comparison. To see that $A_{\omega}$ has stable rank one, let $x \in A_{\omega}$, say $x=\left[\left(x_{n}\right)_{n=1}^{\infty}\right]$. Since $A$ has stable rank one, we find $g_{n} \in A$ such that $\left\|x_{n}-g_{n}\right\|<\frac{1}{n}$ and $g_{n}$ is invertible. Look at the polar decomposition $g_{n}=u_{n}\left|g_{n}\right|$. Let $u=\left[\left(u_{n}\right)_{n=1}^{\infty}\right]$ and $g=\left[\left(g_{n}\right)_{n=1}^{\infty}\right]$. Then

$$
x=\left[\left(x_{n}\right)_{n=1}^{\infty}\right]=\left[\left(g_{n}\right)_{n=1}^{\infty}\right]=u|g|,
$$


where $u \in A_{\omega}$ is a unitary and $|g| \in A_{\omega}$ is positive. Then

$$
x \approx_{\varepsilon} u(|g|+\varepsilon)
$$

and $u(|g|+\varepsilon)$ is invertible.

V.2.6 Remark. To get examples of $\mathrm{C}^{*}$-algebras to which Lemma V.2.5 applies, let us briefly recall a result on limit traces established by Ozawa. In [Oza13, Theorem 8] he shows (in a more general fashion) that

$$
{\overline{T(A)_{\omega}}}^{\text {weak }^{*}}=T\left(A_{\omega}\right)
$$

if $A$ has commutator bounds $(m, C)$ for some $m \in \mathbb{N}$ and $C>0$ in the sense of [NR16, Remark 2.2]:

For every $h \in A_{+}$and $\varepsilon>0$, there exist $x_{1}, y_{1}, \cdots, x_{m}, y_{m} \in A$ such that

$$
\left\|h-\sum_{i=1}^{m}\left[x_{i}, y_{i}\right]\right\| \leq \sup _{\tau \in T(A)} \tau(h)+\varepsilon
$$

and

$$
\sum_{i=1}^{m}\left\|x_{i}\right\|\left\|y_{i}\right\| \leq C\|h\| .
$$

Using [NR16, Proposition 2.4] we know that commutator bounds pass to inductive limits. Since matrix algebras have commutator bounds $(1,2)$ ([Fac82, Lemma 3.5]) it follows that any UHF-algebra has commutator bounds $(1,2)$ and we see that

$$
T(U)_{\omega}=T\left(U_{\omega}\right)
$$

where $U$ is a UHF-algebra. In particular, it follows that $U_{\omega} \in \mathscr{A}$.

V.2.7 Lemma. (cf. Theorem III.1.5) Let $A \in \mathscr{A}$. If $h$ and $k$ are Lebesgue contractions in $A$, then $h \approx_{\mathrm{u}} k$.

Proof. By LemmaV.2.2 we see that $\mathrm{Cu}\left(\phi_{h}\right)=\mathrm{Cu}\left(\phi_{k}\right)$, where $\phi_{h}, \phi_{k}: C_{0}(0,1] \rightarrow$ $A$ denote the ${ }^{*}$-homomorphisms corresponding to $h$ resp. $k$. Furthermore, $\mathrm{Cu}\left(\phi_{h}\right)([\iota])=[h] \leq\left[1_{A}\right]$ and similarly $\mathrm{Cu}\left(\phi_{k}\right)([\iota]) \leq\left[1_{A}\right]$. By Theorem V.1.14 it follows that $\phi_{h} \approx_{\mathrm{u}} \phi_{k}$ and hence $h \approx_{\mathrm{u}} k$.

V.2.8 Lemma. (cf. Lemma III.2.3) Let $\mathfrak{p}$ be a supernatural number and let $\varphi, \psi: M_{\mathfrak{p}} \rightarrow A$ be two c.p.c. order zero maps such that $h_{\varphi}$ and $h_{\psi}$ are Lebesgue contractions. Assume also that $A \in \mathscr{A}$. Then $\varphi \approx_{\mathrm{u}} \psi$. 
Proof. We may assume that $\mathfrak{p}$ is finite, say equal to $p \in \mathbb{N}$. We saw in the proof of Lemma III.2.3 that $\varphi\left(e_{11}\right)$ and $\psi\left(e_{11}\right)$ both have $\frac{1}{p}$ Lebesgue spectral measure. By Lemma|V.2.7 it follows that $\varphi\left(e_{11}\right) \approx \psi\left(e_{11}\right)$. By Lemma III.2.1 and the fact that $A$ has stable rank one it now follows that $\varphi \approx_{\mathrm{u}} \psi$.

V.2.9 Definition. Let $p \in \mathbb{N}$. Fix $\varepsilon>0$ and $\mathcal{F} \subseteq C_{0}(0,1] \otimes M_{p}$ finite. A c.p.c. map $\varphi: C_{0}(0,1] \otimes M_{p} \rightarrow A$ is called

- $(\mathcal{F}, \varepsilon)$-multiplicative if

$$
\|\varphi(x y)-\varphi(x) \varphi(y)\|<\varepsilon \quad(x, y \in \mathcal{F})
$$

- $(\mathcal{F}, \varepsilon)$-standard if

$$
\left|(\tau \circ \varphi)(x)-\tau_{\text {Leb }}(x)\right|<\varepsilon \quad(x \in \mathcal{F}, \tau \in T(A)) .
$$

The following lemma is inspired by [Sch18, Lemma 5.2].

V.2.10 Lemma. Let $p \in \mathbb{N}$ and assume $U$ is a UHF-algebra. Then, for every finite set $\mathcal{F} \subseteq C_{0}(0,1] \otimes M_{p}$ and $\varepsilon>0$, there exists a finite set $\mathcal{G} \subseteq C_{0}(0,1] \otimes$ $M_{p}$ and $\delta>0$ such that the following holds: Whenever $\varphi, \psi: C_{0}(0,1] \otimes M_{p} \rightarrow$ $U$ are $(\mathcal{G}, \delta)$-multiplicative and $(\mathcal{G}, \delta)$-standard c.p.c. maps, there exists a unitary $u \in U$ such that

$$
\left\|u \varphi(f) u^{*}-\psi(f)\right\|<\varepsilon \quad(f \in \mathcal{F}) .
$$

Proof. Assume the result is false. Then, there exist $\mathcal{F} \subseteq C_{0}(0,1] \otimes M_{p}$ finite and $\varepsilon>0$ and c.p.c. maps $\varphi_{n}, \psi_{n}: C_{0}(0,1] \otimes M_{p} \rightarrow U$ which are $\left(\mathcal{G}_{n}, \delta_{n}\right)$ multiplicative and $\left(\mathcal{G}_{n}, \delta_{n}\right)$-standard, where $\mathcal{G}_{n}$ is an increasing sequence of finite subsets of $C_{0}(0,1] \otimes M_{p}$ with dense union and $\delta_{n} \downarrow 0$, but such that for every unitary $u \in U$ we have

$$
\left\|u \varphi_{n}(f) u^{*}-\psi_{n}(f)\right\| \geq \varepsilon
$$

for some $f \in \mathcal{F}$. By assumption, the $\varphi_{n}$ and $\psi_{n}$ induce standard ${ }^{*}$-homomorphisms $\varphi, \psi: C_{0}(0,1] \otimes M_{p} \rightarrow U_{\omega}$. By Lemma V.2.8 and the fact that $U_{\omega} \in \mathscr{A}$, there exists a unitary $u \in U_{\omega}$ with $\operatorname{ad}(u) \circ \varphi=\psi(14$. We can write

\footnotetext{
${ }^{14} \mathrm{As} U_{\omega}$ is an ultrapower, a reindexing argument can be used to turn $\approx_{\mathrm{u}}$ into $\sim_{\mathrm{u}}$, see Remark I.5.2.
} 
$u=\left[\left(u_{n}\right)_{n=1}^{\infty}\right]$ for a sequence of unitaries $u_{n} \in U{ }^{15}$ As

$$
\lim _{n \rightarrow \omega}\left\|u_{n} \varphi_{n}(f) u_{n}^{*}-\psi_{n}(f)\right\|=0 \quad(f \in \mathcal{F}),
$$

there exists for every $f \in \mathcal{F}$ a set $X_{f} \in \omega$ with $\left\|u_{n} \varphi_{n}(f) u_{n}^{*}-\psi_{n}(f)\right\|<\varepsilon$, for $n \in X_{f}$. Now, any $n \in \bigcap_{f \in \mathcal{F}} X_{f} \in \omega$ satisfies

$$
\left\|u_{n} \varphi_{n}(f) u_{n}^{*}-\psi_{n}(f)\right\|<\varepsilon \quad(f \in \mathcal{F}),
$$

contradicting our assumption.

\section{V.3 Existence of order zero maps}

V.3.1 Lemma. Let $p \in \mathbb{N}$ and assume $U$ is a UHF-algebra of infinite type. Then, for every finite set $\mathcal{F} \subseteq C_{0}(0,1] \otimes M_{p}$ and $\varepsilon>0$, there exists a $(\mathcal{F}, \varepsilon)$ multiplicative and $(\mathcal{F}, \varepsilon)$-standard c.p.c. map $\phi: C_{0}(0,1] \otimes M_{p} \rightarrow U$.

Proof. Let $h \in Q$ be a Lebesgue contraction, where $Q$ is the universal UHFalgebra. See the proof of Lemma IV.1.4 for the construction of such a Lebesgue contraction. Since $Q$ is strongly self-absorbing, we may fix an isomorphism $\varphi: Q \otimes Q \rightarrow Q$. After identifying $M_{p}$ with a unital subalgebra of $Q$, we consider $\psi: M_{p} \rightarrow Q: x \mapsto \varphi(h \otimes x)$, which is a c.p.c. order zero map such that $h_{\psi}=\varphi\left(h \otimes 1_{p}\right)$ is a Lebesgue contraction. Next, we look at the following diagram:

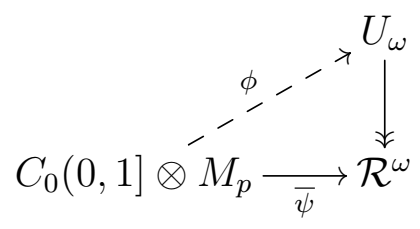

By viewing $U$ as a weak*-dense subalgebra of $\mathcal{R}$, the unique separably acting hyperfinite $\mathrm{II}_{1}$-factor, the inclusion $U \hookrightarrow \mathcal{R}$ induces a trace preserving surjection $U_{\omega} \rightarrow \mathcal{R}^{\omega}$, by [KR14, Theorem 3.3]. To get a ${ }^{*}$-homomorphic lift $\phi$ of $\bar{\psi}{ }^{16}$ we use that the cone over $M_{p}$ is projective. Next, by the Choi-Effros

\footnotetext{
${ }^{15}$ Look at any lift $\left(x_{n}\right)_{n=1}^{\infty}$ of $u$ in $\ell^{\infty}(U)$. Then the $x_{n}$ are approximately unitary and in particular eventually invertible. By replacing $x_{n}$ with $u_{n}:=x_{n}\left|x_{n}\right|^{-1}$ we get a unitary lift.

${ }^{16}$ Since $Q$ has weak closure $\mathcal{R}$ (in its GNS-representation), we regard $\bar{\psi}$ as a map taking values in $\mathcal{R}$, which then sits canonically inside $\mathcal{R}^{\omega}$. Recall also that $\mathcal{R}^{\omega}$ is the tracial ultrapower of $\mathcal{R}$, given by $\ell^{\infty}(\mathcal{R})$ modulo sequences $\left(x_{n}\right)_{n=1}^{\infty}$ such that $\lim _{\omega} \tau_{\mathcal{R}}\left(x_{n}^{*} x_{n}\right)=0$.
} 
lifting theorem, there are c.p.c. maps $\phi_{n}: C_{0}(0,1] \otimes M_{p} \rightarrow U$ such that

$$
\phi(x)=\left[\left(\phi_{n}(x)\right)_{n=1}^{\infty}\right] \quad\left(x \in C_{0}(0,1] \otimes M_{p}\right) .
$$

Then, the $\phi_{n}$ are approximately multiplicative and approximately standard, since the lift $\phi$ is a standard ${ }^{*}$-homomorphism. The result follows.

We can now present two alternative proofs of Lemma IV.1.5.

V.3.2 Lemma. (cf. Lemma IV.1.5) Let $p \in \mathbb{N}$ and let $U$ be a UHF-algebra of infinite type. Then, there exists a c.p.c. order zero map $\psi: M_{p} \rightarrow U$ such that $h_{\psi}$ is a Lebesgue contraction.

Proof using Lemma V.3.1. Let $\left(\mathcal{F}_{n}\right)_{n=1}^{\infty}$ be an increasing sequence of finite subsets of $C_{0}(0,1] \otimes M_{p}$ with dense union and let $\varepsilon_{n}>0$ such that $\sum \varepsilon_{n}<\infty$. Find finite subsets $\mathcal{G}_{n} \subseteq C_{0}(0,1] \otimes M_{p}$ and $\delta_{n}>0$ accordingly to Lemma V.2.10. We may assume that $\delta_{n} \downarrow 0$ and $\left(\mathcal{G}_{n}\right)_{n=1}^{\infty}$ is increasing with dense union. By Lemma V.3.1, for each $n \in \mathbb{N}$, there exists a $\left(\mathcal{G}_{n}, \delta_{n}\right)$-multiplicative and $\left(\mathcal{G}_{n}, \delta_{n}\right)$-standard c.p.c. map $\phi_{n}: C_{0}(0,1] \otimes M_{p} \rightarrow U$. By Lemma V.2.10. for each $n \geq 1$, there exists a unitary $u_{n+1} \in U$ such that

$$
\left\|\phi_{n}(x)-u_{n+1} \phi_{n+1}(x) u_{n+1}^{*}\right\|<\varepsilon_{n} \quad\left(x \in \mathcal{F}_{n}\right) .
$$

Define $\psi_{1}:=\phi_{1}$ and

$$
\psi_{n}:=u_{2} u_{3} \cdots u_{n} \phi_{n}(\cdot) u_{n}^{*} \cdots u_{3}^{*} u_{2}^{*} \quad(n \geq 2) .
$$

Then it is easy to see that $\left(\psi_{n}(x)\right)_{n=1}^{\infty}$ is Cauchy for all $x \in \bigcup_{n=1}^{\infty} \mathcal{F}_{n}$ and hence for all $x \in C_{0}(0,1] \otimes M_{p}$. Finally, define

$$
\psi: C_{0}(0,1] \otimes M_{p} \rightarrow U: x \mapsto \lim _{n \rightarrow \infty} \psi_{n}(x) .
$$

Then $\psi$ is a standard ${ }^{*}$-homomorphism, which is the same as a c.p.c. order zero map $\bar{\psi}: M_{p} \rightarrow U$ such that $h_{\bar{\psi}}$ is a Lebesgue contraction.

Proof using Theorem $V .1 .14$. Let $\Gamma: \mathrm{Cu}(U) \rightarrow V(U) \sqcup(0, \infty]$ be the isomorphism from Corollary V.1.13. We claim that the functional

$$
d_{\tau_{\text {Leb }}}: \mathrm{Cu}\left(C_{0}(0,1] \otimes M_{p}\right) \rightarrow[0, \infty]
$$

is actually a $\mathbf{C u}$-morphism. To this end it suffices to show that it preserves the way below relation. Since $C_{0}(0,1]$ is stably projectionless, we see that

$$
\mathrm{Cu}\left(C_{0}(0,1] \otimes M_{p}\right)=\{0\} \sqcup \mathrm{Cu}\left(C_{0}(0,1] \otimes M_{p}\right)_{+} .
$$


Let $x, y \in \mathrm{Cu}\left(C_{0}(0,1] \otimes M_{p}\right)$ such that $x \ll y$. We may assume that $x, y \neq 0$ (because $0 \ll 0$ in $[0, \infty]$ ). Furthermore, we note that $d_{\tau_{\text {Leb }}}(x)<\infty$ [17 By (V.3.1) and Lemma V.1.9 it follows that there exists $0 \neq z \in \mathrm{Cu}\left(C_{0}(0,1] \otimes\right.$ $\left.M_{p}\right)$ such that $x+z \leq y$. Since $\tau_{\text {Leb }}$ is faithful we see that $d_{\tau_{\text {Leb }}}(z)>0$ and hence

$$
d_{\tau_{\text {Leb }}}(x)<d_{\tau_{\text {Leb }}}(x)+d_{\tau_{\text {Leb }}}(z)=d_{\tau_{\text {Leb }}}(x+z) \leq d_{\tau_{\text {Leb }}}(y) .
$$

The calculation makes sense, because $d_{\tau_{\text {Leb }}}(x)<\infty$.

Let now $\alpha: \mathrm{Cu}\left(C_{0}(0,1] \otimes M_{p}\right) \rightarrow \mathrm{Cu}(U)$ be such that the following diagram commutes:

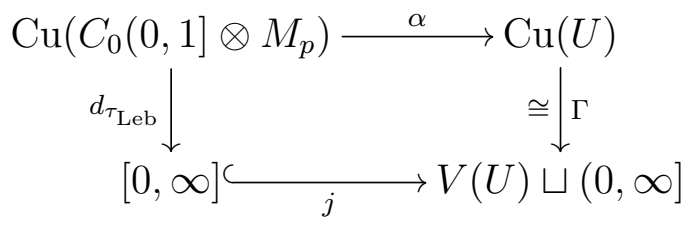

Note that $\alpha\left(\left[\iota \otimes 1_{p}\right]\right)=\Gamma^{-1}\left(1^{\prime}\right) \leq \Gamma^{-1}\left(\left[1_{U}\right]\right)=\left[1_{U}\right]$, where $1^{\prime}$ denotes the element 1 considered in $(0, \infty]$, cf. Example V.1.11. Now Theorem V.1.14 shows that the $\mathbf{C u}$-morphism $\alpha$ lifts to a ${ }^{*}$-homomorphism $\varphi: C_{0}(0,1] \otimes M_{p} \rightarrow$ $U$. Let us show that $\varphi$ is standard. Indeed, denote

$$
\tau:=\tau_{U} \circ \varphi
$$

Then $\tau$ is a trace on $C_{0}(0,1] \otimes M_{p}$ and by Theorem V.1.6 it follows that $\tau=\tau_{\text {Leb }}$ if $d_{\tau}=d_{\tau_{\text {Leb }}}$. Let us check this:

$$
j \circ d_{\tau}=j \circ d_{\tau_{U} \circ \varphi}=j \circ d_{\tau_{U}} \circ \alpha=\Gamma \circ \alpha=j \circ d_{\tau_{\text {Leb }}} .
$$

It follows that $d_{\tau}=d_{\tau_{\mathrm{Leb}}}$, because $j$ is injective.

\section{V.4 Existence and uniqueness of maps on di- mension drop algebras}

Using Robert's classification theorem, we can prove Lemma IV.2.4 immediately, without having to establish existence results for standard c.p.c. order zero maps first.

\footnotetext{
${ }^{17}$ We already noted that $g=\sup _{n} g_{n}$, where each $g_{n}$ lives in some matrix algebra over $C_{0}(0,1]$, see also [APT11, Lemma 5.23]. But then, since $f \ll g$, we have $f \leq g_{n}$ for some $n \in \mathbb{N}$ and the claim follows, because $d_{\tau_{\text {Leb }}}\left(g_{n}\right)<\infty$.
} 
V.4.1 Lemma. (cf. Lemma IV.2.4) Let $\mathfrak{p}$ and $\mathfrak{q}$ be coprime supernatural numbers of infinite type and let $U$ be a UHF-algebra of infinite type. Then, there exists a standard ${ }^{*}$-homomorphism

$$
\varphi: Z_{\mathfrak{p}, \mathfrak{q}} \rightarrow U
$$

which is unique up to approximate unitary equivalence.

Proof. Since $Z_{\mathfrak{p}, \mathfrak{q}}$ has stable rank one (see GLN14, Proposition 3.3]), we have, by Remark V.1.8, that

$$
\mathrm{Cu}\left(Z_{\mathfrak{p}, \mathfrak{q}}\right)=V\left(Z_{\mathfrak{p}, \mathfrak{q}}\right) \sqcup \mathrm{Cu}\left(Z_{\mathfrak{p}, \mathfrak{q}}\right)_{+} \cong \mathbb{N} \sqcup \mathrm{Cu}\left(Z_{\mathfrak{p}, \mathfrak{q}}\right)_{+} \cdot
$$

See Lemma I.3.3 for the computation of $V\left(Z_{\mathfrak{p}, \mathfrak{q}}\right)$. Let us define a map

$$
\alpha: \mathbb{N} \sqcup \mathrm{Cu}\left(Z_{\mathfrak{p}, \mathfrak{q}}\right)_{+} \rightarrow \mathbb{N} \sqcup(0, \infty]
$$

by

$$
\alpha(x):= \begin{cases}x & \text { if } x \in \mathbb{N} \\ d_{\tau_{\text {Leb }}}(x) & \text { if } x \in \mathrm{Cu}\left(Z_{\mathfrak{p}, \mathfrak{q}}\right)_{+}\end{cases}
$$

The set $\mathbb{N} \sqcup(0, \infty]$ has the structure of a $\mathbf{C u}$-semigroup as explained in Example V.1.11. Let us check that $\alpha$ is a $\mathbf{C u}$-morphism.

(M1): Clearly $\alpha$ is additive on each component and preserves the zero element. If $n \in \mathbb{N}$ and $x \in \mathrm{Cu}\left(Z_{\mathfrak{p}, \mathfrak{q}}\right)_{+}$, then $n+x \in \mathrm{Cu}\left(Z_{\mathfrak{p}, \mathfrak{q}}\right)_{+}\left(\right.$since $\mathrm{Cu}\left(Z_{\mathfrak{p}, \mathfrak{q}}\right)_{+}$ is absorbing) and we get

$$
\begin{aligned}
\alpha(n+x) & =d_{\tau_{\mathrm{Leb}}}(n+x)=n d_{\tau_{\mathrm{Leb}}}(1)+d_{\tau_{\mathrm{Leb}}}(x)=n+d_{\tau_{\mathrm{Leb}}}(x) \\
& =\alpha(n)+\alpha(x) .
\end{aligned}
$$

Recall that we identify $\mathbb{N} \cong\left\{n\left[1_{Z_{\mathfrak{p}, \mathfrak{q}}}\right]: n \in \mathbb{N}\right\} \subseteq \mathrm{Cu}\left(Z_{\mathfrak{p}, \mathfrak{q}}\right)$.

(M2): Clearly, $\alpha$ is order preserving on each component. Assume $n \in \mathbb{N}$ and $[f] \in \mathrm{Cu}\left(Z_{\mathfrak{p}, \mathfrak{q}}\right)_{+}$. If $n \leq[f]$, we have to show that $\alpha(n) \leq \alpha([f])$. By definition of $\alpha$ and the order on $\mathbb{N} \sqcup(0, \infty]$, this is true if $n<\mathbb{R} d_{\tau_{\text {Leb }}}([f])$. However, since $n$ is compact ${ }^{18}$, we see that $n \ll[f]$. Then Lemma V.1.9 shows that there exists $0 \neq z \in \mathrm{Cu}\left(Z_{\mathfrak{p}, \mathfrak{q}}\right)$ such that $n+z \leq[f]$. It follows that

$$
n=d_{\tau_{\text {Leb }}}(n)<d_{\tau_{\text {Leb }}}(n)+d_{\tau_{\text {Leb }}}(z) \leq d_{\tau_{\text {Leb }}}([f]) .
$$

\footnotetext{
${ }^{18}$ An element $x \in \mathrm{Cu}(A)$ is called compact if $x \ll x$.
} 
If $[f] \leq n$ it is clear that $\alpha([f]) \leq \alpha(n)$.

(M3): This follows from the fact that $\alpha$ preserves suprema of increasing sequences on each component.

(M4) It remains to prove that $\alpha$ preserves the way below relation. It is enough to check this on $\mathrm{Cu}\left(Z_{\mathfrak{p}, \mathfrak{q}}\right)_{+}$. Again, this follows from Lemma V.1.9.

We see that $\alpha$ is $\mathbf{C u}$-morphism. Using Lemma V.1.13 and composing $\alpha$ with the inclusion

$$
\mathbb{N} \sqcup(0, \infty] \rightarrow V(U) \sqcup(0, \infty] \cong \mathrm{Cu}(U),
$$

it follows by Theorem V.1.15 that $\alpha$ lifts to a unital ${ }^{*}$-homomorphism $\varphi: Z_{\mathfrak{p}, \mathfrak{q}} \rightarrow$ $U \sqrt{19}$ Furthermore, it is again easy to check that $d_{\tau_{U} \circ \varphi}=d_{\tau_{\text {Leb }}}$, showing that $\tau_{U} \circ \varphi=\tau_{\text {Leb }}$, as desired.

The uniqueness statement is also contained in Theorem V.1.15.

\footnotetext{
${ }^{19}$ Note the $\alpha\left(\left[1_{Z_{\mathfrak{p}, \mathfrak{q}}}\right]\right)=\left[1_{U}\right]$.
} 


\section{Chapter VI}

\section{Strongly self-absorbing stationary inductive limits}

In this chapter we show that the stationary inductive limit associated to a unitarily suspended *-homomorphism is strongly self-absorbing (Theorem VI.2.2. We end with some general observations, for example that these limits are all isomorphic and define the initial object in the category of strongly self-absorbing $\mathrm{C}^{*}$-algebras.

\section{VI.1 An approximate intertwining}

VI.1.1 Notation. Let $n \in \mathbb{N}$ and $\mathfrak{p}_{1}, \mathfrak{p}_{2}, \cdots, \mathfrak{p}_{n}$ be supernatural numbers. Let us define

$$
M_{\left(\mathfrak{p}_{1}, \cdots, \mathfrak{p}_{n}\right)}:=M_{\mathfrak{p}_{1}} \otimes M_{\mathfrak{p}_{2}} \otimes \cdots \otimes M_{\mathfrak{p}_{n}}
$$

If $i_{1}, i_{2}, \cdots, i_{k}$ are $k$ distinct integers, not necessarily in increasing order, with $i_{k} \in\{1,2, \cdots, n\}$, then there is an obvious inclusion

$$
l_{\left(\mathfrak{p}_{1}, \cdots, \mathfrak{p}_{n}\right)}^{\left[i_{1}, i_{2}, \cdots, i_{k}\right]}: M_{\left(\mathfrak{p}_{i_{1}}, \mathfrak{p}_{2}, \cdots, \mathfrak{p}_{i_{k}}\right)} \rightarrow M_{\left(\mathfrak{p}_{1}, \cdots, \mathfrak{p}_{n}\right)} .
$$

Let us furthermore denote

$$
M_{\left(\mathfrak{p}_{1}, \cdots, \mathfrak{p}_{n}\right)}^{\left[i_{1}, i_{2}, \cdots, i_{k}\right]}:=\operatorname{Im}\left(l_{\left(\mathfrak{p}_{1}, \cdots, \mathfrak{p}_{n}\right)}^{\left[i_{1}, i_{2}, \cdots, i_{k}\right]}\right) .
$$

If the $n$-tupel $\left(\mathfrak{p}_{1}, \cdots, \mathfrak{p}_{n}\right)$ in question is clear, we will omit the subscripts and simply write $\imath^{\left[i_{1}, \cdots, i_{k}\right]}$ and $M^{\left[i_{1}, \cdots, i_{k}\right]}$. 
VI.1.2 Example. The map $\imath_{(\mathfrak{p}, \mathfrak{q})}^{[1]}: M_{\mathfrak{p}} \rightarrow M_{\mathfrak{p}} \otimes M_{\mathfrak{q}}$ for example is the first factor embedding, whereas $\imath_{(\mathfrak{p}, \mathfrak{q})}^{[2]}: M_{\mathfrak{q}} \rightarrow M_{\mathfrak{p}} \otimes M_{\mathfrak{q}}$ denotes the second factor embedding. Another example is the flip map $M_{\mathfrak{p}} \otimes M_{\mathfrak{p}} \rightarrow M_{\mathfrak{p}} \otimes M_{\mathfrak{p}}$, which is $\imath_{(\mathfrak{p}, \mathfrak{p})}^{[2,1]}$ in our notation.

VI.1.3 Definition. Let $\mathfrak{p}_{1}, \cdots, \mathfrak{p}_{n}$ and $\mathfrak{q}_{1}, \cdots, \mathfrak{q}_{m}$ be supernatural numbers. Let $\mathfrak{r}:=\left(\mathfrak{p}_{1}, \mathfrak{p}_{2}, \cdots, \mathfrak{p}_{n}\right)$ and $\mathfrak{s}:=\left(\mathfrak{q}_{1}, \mathfrak{q}_{2}, \cdots, \mathfrak{q}_{m}\right)$. We then define

$$
Z_{\mathfrak{r}, \mathfrak{s}}=\left\{f \in C\left([0,1], M_{\mathfrak{r}} \otimes M_{\mathfrak{s}}\right): \begin{array}{l}
f(0) \in M_{\mathfrak{r}} \otimes 1_{\mathfrak{s}} \\
f(1) \in 1_{\mathfrak{r}} \otimes M_{\mathfrak{s}}
\end{array}\right\}
$$

VI.1.4 Convention. Let $\mathfrak{p}$ and $\mathfrak{q}$ be supernatural numbers. To simplify notation a bit, let us define $\mathfrak{p}^{2}:=(\mathfrak{p}, \mathfrak{p}), \mathfrak{q}^{2}:=(\mathfrak{q}, \mathfrak{q})$ and $\mathfrak{r}:=\left(\mathfrak{p}^{2}, \mathfrak{q}^{2}, \mathfrak{p}^{2}, \mathfrak{q}^{2}\right)$. We then identify $Z_{\mathfrak{p}^{2}, \mathfrak{q}^{2}} \otimes Z_{\mathfrak{p}^{2}, \mathfrak{q}^{2}}$ with a $\mathrm{C}^{*}$-subalgebra of $C\left([0,1]^{2}, M_{\mathfrak{r}}\right)$, where $f \otimes g \in Z_{\mathfrak{p}^{2}, \mathfrak{q}^{2}} \otimes Z_{\mathfrak{p}^{2}, \mathfrak{q}^{2}}$ gets identified with the function $(f \otimes g)(s, t):=f(s) \otimes$ $g(t)$. Under this identification, one can check that an element $h \in Z_{\mathfrak{p}^{2}, \mathfrak{q}^{2}} \otimes$ $Z_{\mathfrak{p}^{2}, \mathfrak{q}^{2}}$ must satisfy:

- $h(0,0) \in M_{\mathfrak{r}}^{[1,2,5,6]}$,

- $h(s, 0) \in M_{\mathfrak{r}}^{[1,2,3,4,5,6]}$, for $s \in(0,1)$ and

- $h(1,0) \in M_{\mathfrak{r}}^{[3,4,5,6]}$.

Similar boundary conditions must be fulfilled on the remaining sides of the square.

VI.1.5 Notation. Let $\mu: Z_{\mathfrak{p}, \mathfrak{q}} \rightarrow Z_{\mathfrak{p}, \mathfrak{q}}$ be a unital *-homomorphism. For $(i, j) \in\{(1,3),(2,4)\}$ we define

$$
\mu^{[i, j]}: Z_{\mathfrak{p}, \mathfrak{q}} \rightarrow Z_{\mathfrak{p}^{2}, \mathfrak{q}^{2}} ; \quad \mu_{t}^{[i, j]}:=\imath_{\left(\mathfrak{p}^{2}, \mathfrak{q}^{2}\right)}^{[i, j]} \circ \mu_{t}
$$

Furthermore, we define the following *-homomorphism:

$$
\widetilde{\widetilde{\mu}}: Z_{\mathfrak{p}, \mathfrak{q}} \otimes Z_{\mathfrak{p}, \mathfrak{q}} \rightarrow Z_{\mathfrak{p}^{2}, \mathfrak{q}^{2}} ; \quad \widetilde{\tilde{\mu}}_{s}(f \otimes g):=\mu_{s}^{[1,3]}(f) \mu_{s}^{[2,4]}(g)
$$

Note that $\mu_{s}^{[1,3]}$ and $\mu_{s}^{[2,4]}$ are commuting *-homomorphisms.

VI.1.6 Proposition. Let $\mathfrak{p}$ and $\mathfrak{q}$ be coprime supernatural numbers of infinite type and assume $\mu: Z_{\mathfrak{p}, \mathfrak{q}} \rightarrow Z_{\mathfrak{p}, \mathfrak{q}}$ is unitarily suspended. Then

$$
\left(\operatorname{id}_{Z_{\mathfrak{p}^{2}, \mathfrak{q}^{2}}} \otimes 1_{Z_{\mathfrak{p}^{2}, \mathfrak{q}^{2}}}\right) \circ \widetilde{\widetilde{\mu}} \approx_{\mathrm{u}} \mu^{[1,3]} \otimes \mu^{[1,3]}
$$


That is, we can make the following diagram commute arbitrary well up to conjugating by a unitary.

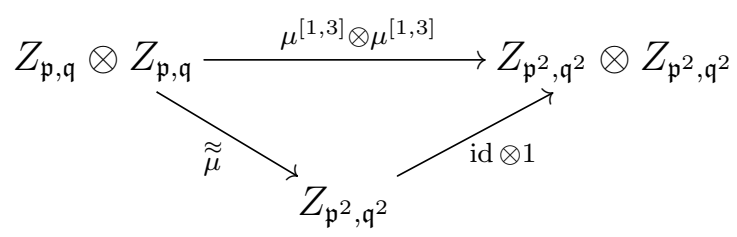

Proof. (I) Let us first do some setup. Recall from Definition IV.2.5 that $\mu: Z_{\mathfrak{p}, \mathfrak{q}} \rightarrow Z_{\mathfrak{p}, \mathfrak{q}}$ is given by

$$
\mu_{t}= \begin{cases}\operatorname{ad}\left(v_{t}\right) \circ\left(\alpha_{0} \otimes 1_{\mathfrak{q}}\right) & \text { if } t \in[0,1), \\ 1_{\mathfrak{p}} \otimes \alpha_{1} & \text { if } t=1,\end{cases}
$$

where $\left(v_{t}\right)_{t \in[0,1)}$ is a unitary path in $M_{\mathfrak{p}} \otimes M_{\mathfrak{q}}$ starting at the identity and where $\alpha_{0}: Z_{\mathfrak{p}, \mathfrak{q}} \rightarrow M_{\mathfrak{p}}$ and $\alpha_{1}: Z_{\mathfrak{p}, \mathfrak{q}} \rightarrow M_{\mathfrak{q}}$ are unital ${ }^{*}$-homomorphisms. During the proof we identify the codomain of $\mu^{[1,3]} \otimes \mu^{[1,3]}$ with functions on the square, accordingly to Convention VI.1.4. Under this identification, the map $\mu^{[1,3]} \otimes \mu^{[1,3]}$ becomes:

$$
\left(\mu^{[1,3]} \otimes \mu^{[1,3]}\right)(f \otimes g)(s, t)=\imath_{\mathfrak{r}}^{[1,3]}\left(\mu_{s}(f)\right) \cdot \iota_{\mathfrak{r}}^{[5,7]}\left(\mu_{t}(g)\right),
$$

where $\mathfrak{r}=\left(\mathfrak{p}^{2}, \mathfrak{q}^{2}, \mathfrak{p}^{2}, \mathfrak{q}^{2}\right)$ is as in Convention VI.1.4. Finally, we will need to write $Z_{\mathfrak{p}, \mathfrak{q}}$ as an inductive $\operatorname{limit} \underset{\lim }{\longrightarrow}\left(Z_{P_{n}, Q_{n}}, \gamma_{n, n+1}\right)$, as explained in Convention IV.2.3.

(II) Let us fix a normalized and finite set $\mathcal{F} \subseteq Z_{\mathfrak{p}, \mathfrak{q}}$ and let $\varepsilon>0$. The goal is to find a unitary $W \in Z_{\mathfrak{p}^{2}, \mathfrak{q}^{2}} \otimes Z_{\mathfrak{p}^{2}, \mathfrak{q}^{2}}$ making the diagram (VI.1.1) commute up to $\varepsilon$ on $\mathcal{F} \otimes \mathcal{F}:=\{f \otimes g: f, g \in \mathcal{F}\}$. For convenience we may assume that the unitary path $\left(v_{t}\right)_{t \in[0,1)}$ is constant in a small open neighborhood of zero, i.e. we may assume there exists $0<\eta<1$ such that $v_{t}=1_{\mathfrak{p q}}$, for $t \in[0, \eta]$. Let us fix some $0<\delta<\eta$, such that

$$
\left|s-s^{\prime}\right| \leq \delta \quad \Rightarrow \quad\left\|\mu_{s}(f)-\mu_{s^{\prime}}(f)\right\| \leq \frac{\varepsilon}{10} \quad(f \in \mathcal{F})
$$

and let $n \in \mathbb{N}$ such that

$$
\left\|\kappa_{n}(\mu(f))-\mu(f)\right\| \leq \frac{\varepsilon}{10} \quad(f \in \mathcal{F}),
$$

where $\kappa_{n}$ is the conditional expectation as defined in Convention IV.2.3. By possibly enlarging $n$ we can ensure the existence $]^{1}$ of a unitary path $\left(\bar{v}_{t}\right)_{t \in[0,1]}$

\footnotetext{
${ }^{1}$ This is done by approximating the unitary $\left(v_{t}\right)_{t \in[0,1-\delta]} \in C\left([0,1-\delta], M_{\mathfrak{p}} \otimes M_{\mathfrak{q}}\right)$ by a unitary in $C\left([0,1-\delta], M_{P_{n}} \otimes M_{Q_{n}}\right)$.
} 
such that

$$
\left[\begin{array}{ll}
\bar{v}_{t}=1_{P_{n}} \otimes 1_{Q_{n}} & \text { if } t \in[0, \delta], \\
\left\|\bar{v}_{t}-v_{t}\right\| \leq \frac{\varepsilon}{10} & \text { if } t \in[\delta, 1-\delta], \\
\bar{v}_{t}=\bar{v}_{1-\delta} & \text { if } t \in[1-\delta, 1] .
\end{array}\right.
$$

Let us denote $p:=P_{n}, q:=Q_{n}$ and $r:=(p, p, q, q, p, p, q, q)$. We then identify $M_{r} \subseteq M_{\mathfrak{r}}$ as unital subalgebra, via our fixed inclusions? ${ }^{2}$. Next, let

$$
S_{p} \in M_{p} \otimes M_{p} \quad \text { and } \quad S_{q} \in M_{q} \otimes M_{q}
$$

denote the self-adjoint flip unitaries. This means $S_{p}(x \otimes y) S_{p}^{*}=y \otimes x$, for all $x, y \in M_{p}$ and similarly for $M_{q}$. Then define

$$
\left[\begin{array}{l}
\widetilde{S}_{p}:=\imath_{r}^{[2,5]}\left(S_{p}\right), \\
\widetilde{S}_{q}:=\imath_{r}^{[4,7]}\left(S_{q}\right), \\
\widetilde{S}:=\widetilde{S}_{p} \widetilde{S}_{q}=\widetilde{S}_{q} \widetilde{S}_{p} \in M_{r}^{[2,4,5,7]}
\end{array}\right.
$$

and for $t \in[0,1]$ we let

$$
\begin{aligned}
& u_{t}:=\imath_{\mathfrak{r}}^{[2,4]}\left(v_{\min (t, 1-\delta)}\right), \\
& \bar{u}_{t}:=\imath_{r}^{[2,4]}\left(\bar{v}_{t}\right) .
\end{aligned}
$$

By VI.1.6) we see that $\left\|u_{t}-\bar{u}_{t}\right\| \leq \frac{\varepsilon}{10}$, for all $t \in[0,1]$. We are now ready to define a first approximation to the desired unitary $W$ :

$$
U:[0,1]^{2} \rightarrow M_{r}:(s, t) \mapsto \widetilde{S} \bar{u}_{t} \bar{u}_{s}^{*}
$$

Note that $U$ does not yet define an element of $Z_{\mathfrak{p}^{2}, \mathfrak{q}^{2}} \otimes Z_{\mathfrak{p}^{2}, \mathfrak{q}^{2}}$. However, let us show that the unitary $U$ makes diagram (VI.1.1) commute approximately, i.e.

$$
U\left(\widetilde{\widetilde{\mu}}(f \otimes g) \otimes 1_{Z_{\mathfrak{p}^{2}, \mathfrak{q}^{2}}}\right) U^{*} \approx_{\frac{\varepsilon}{2}} \mu^{[1,3]}(f) \otimes \mu^{[1,3]}(g) \quad(f, g \in \mathcal{F}) .
$$

First, observe the following identities:
(a) $\operatorname{ad}\left(\widetilde{S}_{p}\right) \circ \imath_{r}^{[2,4]}=\imath_{r}^{[5,4]}$,
(b) $\operatorname{ad}\left(\widetilde{S}_{q}\right) \circ \imath_{r}^{[5,4]}=\imath_{r}^{[5,7]}$,
(c) $\operatorname{ad}\left(\widetilde{S}_{q}\right) \circ \imath_{r}^{[2,4]}=\imath_{r}^{[2,7]}$,

\footnotetext{
${ }^{2}$ Remember that $M_{r}$ and $M_{\mathfrak{r}}$ are a tensor product of eight matrix respectively UHFalgebras, see Notation VI.1.1
} 
(d) $\operatorname{ad}(\widetilde{S}) \circ \imath_{r}^{[2,4]}=\imath_{r}^{[5,7]}$,

(e) $u_{t} u_{s}^{*} \mu_{s}^{[2,4]}(g) u_{s} u_{t}^{*}=\mu_{t}^{[2,4]}(g)$, for any $g \in Z_{\mathfrak{p}, \mathfrak{q}}$ and $s, t \in[0,1-\delta]$.

The identities (a)-(d) are easily proven by looking at elementary tensors, whereas (e) follows from the definition of $\mu$. We are now able to prove VI.1.10). Let $f, g \in \mathcal{F}$ and $(s, t) \in[0,1]^{2}$. Filling in the definitions gives

$$
\begin{array}{r}
U_{s, t}\left(\widetilde{\widetilde{\mu}}(f \otimes g) \otimes 1_{Z_{\mathfrak{p}^{2}, \mathfrak{q}^{2}}}\right)(s, t) U_{s, t}^{*} \\
=\widetilde{S} \bar{u}_{t} \bar{u}_{s}^{*} \iota_{\mathfrak{r}}^{[1,3]}\left(\mu_{s}(f)\right) \iota_{\mathfrak{r}}^{[2,4]}\left(\mu_{s}(g)\right) \bar{u}_{s} \bar{u}_{t}^{*} \widetilde{S}^{*} .
\end{array}
$$

Note that $\bar{u}_{t}$ and $\widetilde{S}$ commute with $M_{\mathfrak{r}}^{[1,3]}$. For $(s, t) \in[0,1-\delta]^{2}$, for example, one checks that (VI.1.11) becomes

$$
\begin{aligned}
& \imath_{\mathfrak{r}}^{[1,3]}\left(\mu_{s}(f)\right) \widetilde{S} \bar{u}_{t} \bar{u}_{s}^{*} l_{\mathfrak{r}}^{[2,4]}\left(\mu_{s}(g)\right) \bar{u}_{s} \bar{u}_{t}^{*} \widetilde{S}^{*} \\
& \underset{\frac{2 \varepsilon}{10}}{\stackrel{\text { VI.1.6 }}{\approx}} \quad i_{\mathfrak{r}}^{[1,3]}\left(\mu_{s}(f)\right) \widetilde{S} u_{t} u_{s}^{*} \imath_{\mathfrak{r}}^{[2,4]}\left(\mu_{s}(g)\right) u_{s} u_{t}^{*} \widetilde{S}^{*} \\
& \stackrel{(\mathrm{e})}{=} \quad i_{\mathfrak{r}}^{[1,3]}\left(\mu_{s}(f)\right) \widetilde{S} \tau_{\mathfrak{r}}^{[2,4]}\left(\mu_{t}(g)\right) \widetilde{S}^{*}
\end{aligned}
$$

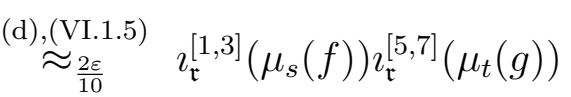

$$
\begin{aligned}
& \stackrel{\text { VI.1.3 }}{=}\left(\mu^{[1,3]}(f) \otimes \mu^{[1,3]}(g)\right)(s, t) \text {. }
\end{aligned}
$$

For $(s, t) \in([1-\delta, 1] \times[0,1-\delta]) \cup([0,1-\delta] \times[1-\delta, 1]) \cup[1-\delta, 1]^{2}$ one proceeds similarly and uses in particular (VI.1.4). This proves the claim (VI.1.10).

(III) The next step is to perturb $U$ on the boundary. Since $S_{p}$ and $S_{q}$ are connected to the identity in $M_{p} \otimes M_{p}$ respectively $M_{q} \otimes M_{q}$ we can find a unitary path $\left(\nu_{t}\right)_{t \in[0,1]}$ inside $M_{r}$ such that the following holds:

$$
\left[\begin{array}{ll}
\nu_{0}=\widetilde{S}_{q} & \\
\nu_{t} \in \mathrm{C}^{*}\left(\widetilde{S}_{q}\right) \subseteq M_{r}^{[4,7]} & \text { if } t \in[0, \delta] \\
\nu_{t}=1_{r} & \text { if } t \in[\delta, 1-\delta] \\
\nu_{t} \in \mathrm{C}^{*}\left(\widetilde{S}_{p}\right) \subseteq M_{r}^{[2,5]} & \text { if } t \in[1-\delta, 1] \\
\nu_{1}=\widetilde{S}_{p} . &
\end{array}\right.
$$

Observe that

(f) $\left[\widetilde{S}, \nu_{t}\right]=0$, for every $t \in[0,1]$,

(g) $\left[\nu_{s}, \imath_{\mathfrak{r}}^{[i, j]}\left(\mu_{s}(f)\right)\right] \approx_{\frac{\varepsilon}{10}} 0$, for all $s \in[0,1],(i, j) \in\{(2,4),(5,7)\}$ and $f \in \mathcal{F}$. 
For (f), let $t \in[0, \delta]$. Then, $\nu_{t} \in M_{r}^{[4,7]}$. Since $\widetilde{S}_{p} \in M_{r}^{[2,5]}$ we see that $\widetilde{S} \nu_{t} \widetilde{S}^{*}=\widetilde{S}_{q} \nu_{t} \widetilde{S}_{q}^{*}$. Note that $\widetilde{S}_{q}$ commutes with $\nu_{t}$ for $t \in[0, \delta]$, since the $\mathrm{C}^{*}$-algebra generated by $\widetilde{S}_{q}$ is commutative. For $t \in[\delta, 1-\delta]$ nothing is to check and for $t \in[1-\delta, 1]$ we argue similarly as before. Let us check (g) for $(i, j)=(2,4)$ and $s \in[0, \delta]$ :

$$
\nu_{s} \imath_{\mathfrak{r}}^{[2,4]}\left(\mu_{s}(f)\right) \nu_{s}^{*} \stackrel{\underset{\text { VI.1.4. }}{\approx}}{\frac{\varepsilon}{10}} \nu_{s} l_{\mathfrak{r}}^{[2,4]}\left(\mu_{0}(f)\right) \nu_{s}^{*}=i_{\mathfrak{r}}^{[2,4]}\left(\mu_{0}(f)\right) .
$$

The last equality follows from the fact that $\mu_{0}(f)=\alpha_{0}(f) \otimes 1_{\mathfrak{q}} \in M_{\mathfrak{p}} \otimes 1_{\mathfrak{q}}$ so that $\imath_{\mathfrak{r}}^{[2,4]}\left(\mu_{0}(f)\right) \in M_{\mathfrak{r}}^{[2]}$, whereas $\nu_{s} \in M_{\mathfrak{r}}^{[4,7]}$ for $s \in[0, \delta]$. A similar observation applies to the case $s \in[1-\delta, 1]$. We can now define the desired unitary $W$ by

$$
W_{s, t}:= \begin{cases}\nu_{\min (s, t)} U_{s, t} & \text { if }(s, t) \in[0, \delta]^{2}, \\ \nu_{\max (s, t)} U_{s, t} & \text { if }(s, t) \in[1-\delta, 1]^{2}, \\ \nu_{t} U_{s, t} \nu_{s} & \text { else. }\end{cases}
$$

This should be compared to the construction of Jiang and Su in [JS99, Proposition 8.3]. Now, we have to check the following:

(i) $W$ is continuous,

(ii) $W \in Z_{\mathfrak{p}^{2}, \mathfrak{q}^{2}} \otimes Z_{\mathfrak{p}^{2}, \mathfrak{q}^{2}}$,

(iii) $\operatorname{ad}(W) \circ\left(\widetilde{\widetilde{\mu}} \otimes 1_{Z_{\mathfrak{p}^{2}, \mathfrak{q}^{2}}}\right) \approx_{(\mathcal{F} \otimes \mathcal{F}, \varepsilon)} \mu^{[1,3]} \otimes \mu^{[1,3]}$.

Without reference, we will use that

$$
U_{s, t}=\widetilde{S} \quad\left((s, t) \in[0, \delta]^{2} \cup[1-\delta, 1]^{2}\right) .
$$

We note that (i) follows easily from (f). In order to show (ii), we have to verify that $W$ satisfies all boundary conditions, as explained in Convention VI.1.4. The computation is not difficult but tedious, so as an example we show how to handle the case $t=0$, which splits up into the following three cases:

- $s \in[0, \delta]: W_{s, t}=\nu_{\min (s, t)} U_{s, t}=\nu_{0} \widetilde{S}=\widetilde{S}_{q} \widetilde{S}=\widetilde{S}_{p} \in M_{r}^{[2,5]}$,

- $s \in[\delta, 1]: W_{s, t}=\nu_{t} U_{s, t} \nu_{s}=\nu_{0} U_{s, 0} \nu_{s}=\widetilde{S}_{q}\left(\widetilde{S} \bar{u}_{s}^{*}\right) \nu_{s}=\widetilde{S}_{p} \bar{u}_{s}^{*} \nu_{s}$, which lives in $M_{r}^{[2,5]} M_{r}^{[2,4]} M_{r}^{[2,5]} \subseteq M_{r}^{[2,4,5]}$, 


$$
\text { - } s=1: W_{s, t}=\widetilde{S}_{p} \bar{u}_{s}^{*} \nu_{s}=\widetilde{S}_{p} \bar{u}_{1-\delta}^{*} \widetilde{S}_{p} \in \widetilde{S}_{p} M_{r}^{[2,4]} \widetilde{S}_{p} \underset{(\mathrm{a})}{\subseteq} M_{r}^{[4,5]} .
$$

It follows that $W$ is a unitary in $Z_{\mathfrak{p}^{2}, \mathfrak{q}^{2}} \otimes Z_{\mathfrak{p}^{2}, \mathfrak{q}^{2}}$ and it remains to prove (iii). For $(s, t) \in[0, \delta]^{2}$ and $f, g \in \mathcal{F}$ we compute

$$
\begin{aligned}
& W_{s, t}\left(\tilde{\tilde{\mu}}(f \otimes g) \otimes 1_{Z_{\mathrm{p}^{2}, \mathfrak{q}^{2}}}\right)(s, t) W_{s, t}^{*} \\
& =\quad \nu_{\min (s, t)} \widetilde{S} l_{\mathfrak{r}}^{[1,3]}\left(\mu_{s}(f)\right) \nu_{\mathfrak{r}}^{[2,4]}\left(\mu_{s}(g)\right) \nu_{\min (s, t)}^{*} \widetilde{S}^{*} \\
& \text { (f), } \\
& \stackrel{(\mathrm{g})}{\approx}_{\frac{\varepsilon}{10}} \quad \widetilde{S}_{\mathfrak{r}}^{[1,3]}\left(\mu_{s}(f)\right) \imath_{\mathfrak{r}}^{[2,4]}\left(\mu_{s}(g)\right) \widetilde{S}^{*} \\
& =U_{s, t}\left(\tilde{\widetilde{\mu}}(f \otimes g) \otimes 1_{Z_{\mathfrak{p}^{2}, \mathfrak{q}^{2}}}\right)(s, t) U_{s, t}^{*} \\
& \underset{\frac{\varepsilon}{2}}{\stackrel{\text { VI.1.10 }}{\approx}}\left(\mu^{[1,3]}(f) \otimes \mu^{[1,3]}(g)\right)(s, t) .
\end{aligned}
$$

The computation for $(s, t) \in[1-\delta, 1]^{2}$ is similar. For the remaining part, we get

$$
\begin{aligned}
& W_{s, t}\left(\tilde{\widetilde{\mu}}(f \otimes g) \otimes 1_{Z_{\mathfrak{p}^{2}, \mathfrak{q}^{2}}}\right)(s, t) W_{s, t}^{*} \\
& =\quad \nu_{t} U_{s, t} \nu_{s} \imath_{\mathfrak{r}}^{[1,3]}\left(\mu_{s}(f)\right) \imath_{\mathfrak{r}}^{[2,4]}\left(\mu_{s}(g)\right) \nu_{s}^{*} U_{s, t}^{*} \nu_{t}^{*} \\
& \stackrel{(\mathrm{g})}{\approx}_{\frac{2 \varepsilon}{10}} \nu_{t} U_{s, t} \iota_{\mathfrak{r}}^{[1,3]}\left(\mu_{s}(f)\right) \iota_{\mathfrak{r}}^{[2,4]}\left(\mu_{s}(g)\right) U_{s, t}^{*} \nu_{t}^{*} \\
& \stackrel{\text { VI.1.10 }}{\approx \frac{\varepsilon}{2}} \nu_{t} \nu_{\mathfrak{r}}^{[1,3]}\left(\mu_{s}(f)\right) \imath_{\mathfrak{r}}^{[5,7]}\left(\mu_{t}(g)\right) \nu_{t}^{*} \\
& \stackrel{(\mathrm{g})}{\approx}{ }_{\frac{2 \varepsilon}{10}} l_{\mathfrak{r}}^{[1,3]}\left(\mu_{s}(f)\right) \imath_{\mathfrak{r}}^{[5,7]}\left(\mu_{t}(g)\right) \\
& \stackrel{\text { VI.1.3 }}{=}\left(\mu^{[1,3]}(f) \otimes \mu^{[1,3]}(g)\right)(s, t) \text {. }
\end{aligned}
$$

This finishes the proof.

\section{VI.2 Proof of the main theorem}

VI.2.1 Definition. Let $\mathfrak{p}$ and $\mathfrak{q}$ be coprime supernatural numbers of infinite type. By Theorem IV.2.6, there exists a unitarily suspended standard *homomorphism $\mu: Z_{\mathfrak{p}, \mathfrak{q}} \rightarrow Z_{\mathfrak{p}, \mathfrak{q}}$, which we fix for the rest of this section. Define

$$
\bar{\mu}: Z_{\mathfrak{p}, \mathfrak{q}} \rightarrow Z_{\mathfrak{p}, \mathfrak{q}} ; \quad \bar{\mu}:=\Phi \circ \mu^{[1,3]},
$$

where $\Phi: Z_{\mathfrak{p}^{2}, \mathfrak{q}^{2}} \rightarrow Z_{\mathfrak{p}, \mathfrak{q}}$ is the canonical isomorphism, induced fiberwise by isomorphisms $M_{\mathfrak{p}^{2}} \rightarrow M_{\mathfrak{p}}$ and $M_{\mathfrak{q}^{2}} \rightarrow M_{\mathfrak{q}}$. We then define

$$
\overrightarrow{Z_{\mathfrak{p}, \mathfrak{q}}^{\mu}}:=\underline{\lim }\left(Z_{\mathfrak{p}, \mathfrak{q}}, \bar{\mu}\right) \text {. }
$$


That is, $\overrightarrow{Z_{\mathfrak{p}, \mathfrak{q}}^{\mu}}$ is the stationary inductive limit of $Z_{\mathfrak{p}, \mathfrak{q}}$ along $\bar{\mu}$.

VI.2.2 Theorem. The $\mathrm{C}^{*}$-algebra $\overrightarrow{Z_{\mathfrak{p}, \mathfrak{q}}^{\mu}}$ is strongly self-absorbing. Furthermore, for any sequence $\left(\varphi_{n}\right)_{n=1}^{\infty}$ of standard ${ }^{*}$-endomorphisms of $Z_{\mathfrak{p}, \mathfrak{q}}$ we have

$$
\overrightarrow{Z_{\mathfrak{p}, \mathfrak{q}}^{\mu}} \cong \underset{\lim }{\longrightarrow}\left(Z_{\mathfrak{p}, \mathfrak{q}}, \varphi_{n}\right) .
$$

Proof. Let us consider the following diagram, where $A:=\underline{\lim }\left(Z_{\mathfrak{p}, \mathfrak{q}}, \varphi_{n}\right)$ :

(I)

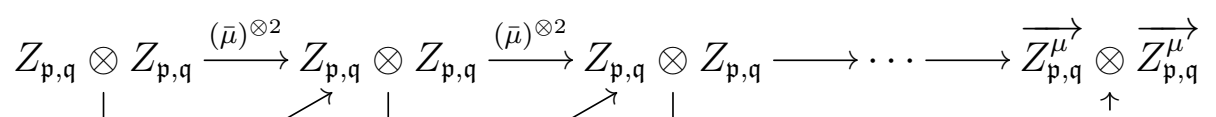

(II)

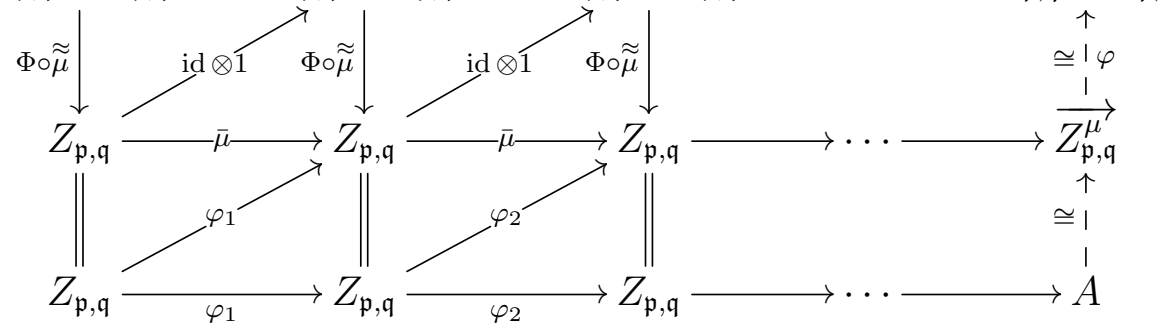

Let us first have a look at row (I). By Proposition VI.1.6, we have (id $\otimes 1$ ) ○ $\widetilde{\tilde{\mu}} \approx_{\mathrm{u}} \mu^{[1,3]} \otimes \mu^{[1,3]}$. By composing with $\Phi \otimes \Phi$ it follows that

$$
(\mathrm{id} \otimes 1) \circ(\Phi \circ \widetilde{\mu}) \approx_{\mathrm{u}} \bar{\mu} \otimes \bar{\mu} .
$$

Furthermore, one easily computes that

$$
(\Phi \circ \tilde{\widetilde{\mu}}) \circ(\mathrm{id} \otimes 1)=\Phi \circ \mu^{[1,3]}=\bar{\mu} .
$$

This shows that each triangle in (I) commutes approximately up to conjugating by a unitary. By $\mathrm{Rør} 02$, Corollary 2.3.3] it follows that there exist unitaries $\left(u_{n}\right)_{n=1}^{\infty}$ in $Z_{\mathfrak{p}, \mathfrak{q}} \otimes Z_{\mathfrak{p}, \mathfrak{q}}$ such that if id $\otimes 1$ is replaced by $\operatorname{ad}\left(u_{n}\right) \circ(\mathrm{id} \otimes 1)$, then the above diagram is an approximate intertwining (in the sense of Rør02, Definition 2.3.1]). By the same corollary the induced isomorphism $\varphi: \overrightarrow{Z_{\mathfrak{p}, \mathfrak{q}}^{\mu}} \rightarrow \overrightarrow{Z_{\mathfrak{p}, \mathfrak{q}}^{\mu}} \otimes \overrightarrow{Z_{\mathfrak{p}, \mathfrak{q}}^{\mu}}$ satisfies

$$
\varphi(x)=\lim _{n \rightarrow \infty} u_{n}(x \otimes 1) u_{n}^{*} \quad\left(x \in \overrightarrow{Z_{\mathfrak{p}, \mathfrak{q}}^{\mu}}\right)
$$

where now each $u_{n}$ is considered as an element of $\overrightarrow{Z_{\mathfrak{p}, \mathfrak{q}}^{\mu}} \otimes \overrightarrow{Z_{\mathfrak{p}, \mathfrak{q}}^{\mu}}$. By definition, it follows that $\overrightarrow{Z_{\mathfrak{p}, \mathfrak{q}}^{\mu}}$ is strongly self-absorbing.

Next, we look at row (II). Clearly, all lower triangles commute and by Theorem III.6.1 we have that $\varphi_{n} \approx_{\mathrm{u}} \bar{\mu}$, for each $n \in \mathbb{N}^{3}$. Again, by an approximate intertwining argument, it follows that $A \cong \overline{Z_{\mathfrak{p}, \mathfrak{q}}^{\mu}}$.

\footnotetext{
${ }^{3}$ Note that $\bar{\mu}$ is still standard.
} 
VI.2.3 Remark. The previous theorem picks up the spirit of [RW10, Theorem 3.4] and shows that any stationary inductive limit with a standard (hence trace collapsing) *-endomorphism is strongly self-absorbing. In our approach however, we do not have to compare the limit to the Jiang-Su algebra $\mathcal{Z}$.

VI.2.4 Lemma. Let $n \in \mathbb{N}$. Then, there exists $k \in \mathbb{N}$ and a unital ${ }^{*}$-homomorphism $Z_{n, n+1} \rightarrow Z_{2^{k}, 2^{k}+1}$.

Proof. Choose $k \in \mathbb{N}$ such that $2^{k} \geq n(n+1)$ and write $2^{k}=n d+r$, where $r \in\{0,1, \cdots, n-1\}$. Then clearly $d \geq n+1>r+1$. By [Sat10, Proposition 2.1], there exist elements $s, c_{1}, c_{2}, \cdots, c_{2^{k}}$ in $Z_{2^{k}, 2^{k}+1}$ such that

$$
c_{1} \geq 0, \quad c_{i} c_{j}^{*}=\delta_{i, j} c_{1}^{2}, \quad s^{*} s+\sum_{j=1}^{2^{k}} c_{j}^{*} c_{j}=1 \text { and } c_{1} s=s .
$$

For $i=1,2, \cdots, n$ define

$$
x_{i}:=\sum_{j=(i-1) \cdot d+1}^{i \cdot d} c_{j} \quad \text { and } \quad b_{i}:=x_{i}^{*} x_{i}=\sum_{j=(i-1) \cdot d+1}^{i \cdot d} c_{j}^{*} c_{j} .
$$

Note that the $b_{i}$ are mutually equivalent orthogonal positive elements. By the relations on the $c_{j}$ and $s$ it is easy to see that

(a) $1=b_{1}+b_{2}+\cdots+b_{n}+\left(c_{n d+1}^{*} c_{n d+1}+\cdots+c_{n d+r}^{*} c_{n d+r}\right)+s^{*} s$,

(b) $\left[s^{*} s\right] \leq\left[c_{1}\right]$ and $\left[c_{1}\right]=\left[c_{1}^{2}\right]=\left[c_{1}^{*} c_{1}\right]$,

(c) $d\left[c_{1}\right]=\left[b_{1}\right]$.

Here $[a]$ denotes the class of an element $a$ in $W\left(Z_{2^{k}, 2^{k}+1}\right)$, the Cuntz semigroup of $Z_{2^{k}, 2^{k}+1}$. Now we have

$$
\begin{aligned}
{\left[1-\left(b_{1}+\cdots+b_{n}\right)\right] } & \stackrel{(\mathrm{a})}{=}\left[\left(c_{n d+1}^{*} c_{n d+1}+\cdots+c_{n d+r}^{*} c_{n d+r}\right)+s^{*} s\right] \\
& \leq\left[c_{n d+1}^{*} c_{n d+1}\right]+\cdots+\left[c_{n d+r}^{*} c_{n d+r}\right]+\left[s^{*} s\right] \\
& =r\left[c_{1}^{*} c_{1}\right]+\left[s^{*} s\right] \stackrel{(\mathrm{b})}{\leq} r\left[c_{1}^{*} c_{1}\right]+\left[c_{1}\right]=(r+1)\left[c_{1}\right] \\
& \ll d\left[c_{1}\right] \stackrel{(\mathrm{c})}{=}\left[b_{1}\right] .
\end{aligned}
$$

The last line follows since $r+1<d$. Hence, there exists $\varepsilon>0$ such that

$$
1-\left(b_{1}+\cdots+b_{n}\right) \lesssim\left(b_{1}-\varepsilon\right)_{+} .
$$

By [RW10, Proposition 5.1 (ii)], it follows that $Z_{n, n+1}$ embeds unitally into $Z_{2^{k}, 2^{k}+1}$. 
VI.2.5 Proposition. Let $p, q \in \mathbb{N}$ be coprime. Then $Z_{p, q}$ maps unitally into any strongly self-absorbing $\mathrm{C}^{*}$-algebra.

Proof. Let $p, q \in \mathbb{N}$ be coprime. We can find $k, l \in \mathbb{N}$ such that $l q-k p=1$ and $2 l>p, 2 k>q$. With $n:=k p$, there exists by [JS99, Proposition 2.5] a unital *-homomorphism $Z_{p, q} \rightarrow Z_{k p, l q}=Z_{n, n+1}$. By Lemma VI.2.4. $Z_{n, n+1}$ embeds unitally into $Z_{2^{k}, 2^{k}+1}$, for some large enough $k$. Finally, by [Win11, Theorem 3.1], $Z_{2^{k}, 2^{k}+1}$ maps unitally into any strongly self-absorbing $\mathrm{C}^{*}$-algebra.

VI.2.6 Lemma. Let $A$ be a strongly self-absorbing $\mathrm{C}^{*}$-algebra that is locally approximated by prime dimension drop algebras. Then $A$ embeds unitally into any strongly self-absorbing $\mathrm{C}^{*}$-algebra.

Proof. Let $D$ be a strongly self-absorbing $\mathrm{C}^{*}$-algebra. Since $A$ is locally approximated by prime dimension drop algebras $Z_{p, q}$ with $p, q \in \mathbb{N}$, it follows by [Lor93, Theorem 3.8] that $A$ is an inductive limit of such dimension drop algebras. By Proposition VI.2.5, each of these embed unitally into $D$ and hence into the central sequence algebra $D_{\omega} \cap D^{\prime}$, see [TW07, Theorem 2.2]. By [TW08, Proposition 2.2 $]^{5}$ we see that $D \cong A \otimes D$ and hence $A$ embeds unitally into $D$.

For the next theorem we first recall Kirchberg's definition of a central sequence algebra (cf. [Kir06, Definition 1.1]).

VI.2.7 Definition. Let $A$ be a $\mathrm{C}^{*}$-algebra. We then define

$$
F(A):=\frac{A_{\omega} \cap A^{\prime}}{\operatorname{Ann}\left(A, A_{\omega}\right)}
$$

where $\operatorname{Ann}\left(A, A_{\omega}\right):=\left\{x \in A_{\omega}: x A=A x=\{0\}\right\}$.

VI.2.8 Remark. If $A$ is $\sigma$-unital and $\left(h_{n}\right)_{n=1}^{\infty}$ is an approximate unit for $A$, then $F(A)$ is unital with unit $\left[\left(h_{n}\right)_{n=1}^{\infty}\right]+\operatorname{Ann}\left(A, A_{\omega}\right)$.

VI.2.9 Theorem. Let $\mathfrak{p}$ and $\mathfrak{q}$ be coprime supernatural numbers of infinite type and let $\left(\varphi_{n}\right)_{n=1}^{\infty}$ be a sequence of standard ${ }^{*}$-endomorphisms of $Z_{\mathfrak{p}, \mathfrak{q}}$. Let us denote

$$
A:=\underline{\lim }\left(Z_{\mathfrak{p}, \mathfrak{q}}, \varphi_{n}\right) .
$$

\footnotetext{
${ }^{4}$ By definition, this means that there exists a sequence $A_{n} \subseteq A$ of prime dimension drop algebras, such that for any finite set $\mathcal{F} \subseteq A$ and $\varepsilon>0$, there exists some $n \in \mathbb{N}$ such that $\mathcal{F} \subseteq_{\varepsilon} A_{n}$, i.e. for every $x \in \mathcal{F}$ there exists $y \in A_{n}$ such that $\|x-y\|<\varepsilon$.

${ }^{5}$ In this proposition, the assumption of $\mathrm{K}_{1}$-injectivity is superfluous.
} 
Then the following holds:

(i) $A$ is the initial object in the category ${ }^{6}$ of strongly self-absorbing $\mathrm{C}^{*}$ algebras,

(ii) If $B$ is any separable $\mathrm{C}^{*}$-algebra such that there exists a unital *-homomorphism $Z_{2,3} \rightarrow F(B)$, then $B \cong B \otimes A$,

(iii) If $B$ is any separable $\mathrm{C}^{*}$-algebra and $Z_{2,3}$ maps unitally into $M(B)_{\omega} \cap B^{\prime}$, then $B \cong B \otimes A$.

Proof. (i) By Theorem VI.2.2 it follows that $A$ is strongly self-absorbing. Then Lemma VI.2.6 implies that $A$ is the initial object in the category of strongly self-absorbing $\mathrm{C}^{*}$-algebras.

(ii) By [Kir06, Corollary 1.13], there is a unital *-homomorphism $Z_{2,3}^{\otimes \infty} \rightarrow$ $F(B)$ and hence any dimension drop algebra $Z_{2^{n}, 3^{n}} \subseteq Z_{2,3}^{\otimes n}$ maps unitally into $F(B)$. Let $(\mathfrak{r}, \mathfrak{s}):=\left(2^{\infty}, 3^{\infty}\right)$. By a similar argument as in [TW08, Proposition 2.2], it follows that $\overrightarrow{Z_{\mathfrak{r}, \mathfrak{s}}^{\mu}}$ (see Definition VI.2.1) maps unitally into $F(B)$. By [Jia97], the algebra $\overrightarrow{Z_{\mathfrak{r}, \mathfrak{s}}^{\mu}}$ is $\mathrm{K}_{1}$-injective]. Then the same proof as in [Naw13, Proposition 5.1] applies to show that $B \cong B \otimes \overrightarrow{Z_{\mathfrak{r}, \mathfrak{s}}^{\mu}}$. See also [Kir06, Proposition 4.11 (1)]. By (i) we know that $A \cong \overrightarrow{Z_{\mathfrak{r}, \mathfrak{s}}^{\mu}}$ and hence $B \cong B \otimes A$.

(iii) Note that there is a unital *-homomorphism

$$
M(B)_{\omega} \cap B^{\prime} \rightarrow F(B):\left[\left(x_{n}\right)_{n=1}^{\infty}\right] \mapsto\left[\left(x_{n} h_{n}\right)_{n=1}^{\infty}\right]+\operatorname{Ann}\left(B, B_{\omega}\right),
$$

where $\left(h_{n}\right)_{n=1}^{\infty}$ is an approximate unit for $B$. It follows that $Z_{2,3}$ maps unitally into $F(B)$ and (ii) applies.

VI.2.10 Remark. It is now particularly easy to see that any prime dimension drop algebra $Z_{\mathfrak{p}, \mathfrak{q}}$ embeds unitally into any $A$, where $A$ is as in Theorem VI.2.9, Furthermore, it is encoded in the construction, that these embeddings are standard.

\footnotetext{
${ }^{6}$ The morphisms in this category are approximate unitary equivalence classes of *homomorphisms. This ensures that the initial object is unique, cf. [TW07 Proposition $5.12]$.

${ }^{7}$ The result there is stated for the Jiang-Su algebra $\mathcal{Z}$, however it only uses that $\mathcal{Z}$ is a simple inductive limit of prime dimension drop algebras.
} 


\title{
List of Symbols
}

$A, B, C, \cdots$
$M_{n}$
$1_{n}$
$\operatorname{Tr}_{n}$
$M_{\mathfrak{p}}$
$1_{\mathfrak{p}}$
$\tau_{M_{\mathfrak{p}}}$
$C_{0}(X)$
$A_{+}$
$A^{+}$
$T(A)$
$\varphi^{*}$
$\sim_{\mathrm{u}}$
$\approx_{\mathrm{u}}$
$\sim$
$\approx$
$\sim_{\mathrm{asu}}$
$\preccurlyeq$
$\sim_{\mathrm{Cu}}$
$x \approx_{\varepsilon} y$
$[x, y]$
$\mathrm{ad}$
$\grave{\imath}, \grave{\imath}, \grave{\varphi}, \grave{\varphi}$
$\sigma(x)$

\author{
$\mathrm{C}^{*}$-algebras \\ complex $n \times n$ matrices \\ unit of $M_{n}$ \\ the unnormalized trace on $M_{n}$ \\ UHF-algebra associated to $\mathfrak{p}$ \\ unit of $M_{\mathfrak{p}}$ \\ the unique tracial state on $M_{\mathfrak{p}}$ \\ cts. functions on $X$ that vanish at infinity \\ positive elements \\ forced unitization \\ tracial state space \\ induced map on the tracial state spaces \\ unitary equivalence \\ approximate unitary equivalence \\ Murray-von Neumann equivalence \\ Approximate Murray-von Neumann equivalence \\ strong asymptotic unitary equivalence \\ Cuntz below \\ Cuntz equivalent \\ $\|x-y\|<\varepsilon$ \\ the commutator $x y-y x$ \\ $\operatorname{ad}(u)(x)=u x u^{*}$ \\ Definition I.3.6 \\ spectrum of an element $x$
}




\section{Bibliography}

[APT11] Pere Ara, Francesc Perera, and Andrew S. Toms. K-theory for operator algebras. Classification of $\mathrm{C}^{*}$-algebras. In Aspects of operator algebras and applications, volume 534 of Contemp. Math., pages 1-71. Amer. Math. Soc., Providence, RI, 2011.

[BBS $\left.{ }^{+} 19\right]$ Joan Bosa, Nathanial P. Brown, Yasuhiko Sato, Aaron Tikuisis, Stuart White, and Wilhelm Winter. Covering dimension of $\mathrm{C}^{*}$ algebras and 2-coloured classification. Mem. Amer. Math. Soc., 257(1233):vii+97, 2019.

[BEEK98] Ola Bratteli, George A. Elliott, David E. Evans, and Akitaka Kishimoto. Homotopy of a pair of approximately commuting unitaries in a simple $\mathrm{C}^{*}$-algebra. J. Funct. Anal., 160(2):466-523, 1998.

[Bla98] Bruce Blackadar. K-theory for operator algebras, volume 5 of Mathematical Sciences Research Institute Publications. Cambridge University Press, Cambridge, second edition, 1998.

[BO08] Nathanial P. Brown and Narutaka Ozawa. C*-algebras and finitedimensional approximations, volume 88 of Graduate Studies in Mathematics. American Mathematical Society, Providence, RI, 2008.

[CE08] Alin Ciuperca and George A. Elliott. A remark on invariants for $\mathrm{C}^{*}$-algebras of stable rank one. Int. Math. Res. Not. IMRN, (5):Art. ID rnm 158, 33, 2008.

[CEI08] Kristofer T. Coward, George A. Elliott, and Cristian Ivanescu. The Cuntz semigroup as an invariant for $\mathrm{C}^{*}$-algebras. J. Reine Angew. Math., 623:161-193, 2008. 
[CET $\left.{ }^{+} 19\right]$ Jorge Castillejos, Samuel Evington, Aaron Tikuisis, Stuart White, and Wilhelm Winter. Nuclear dimension of simple $\mathrm{C}^{*}$-algebras. arxiv:1901.05853 [math.OA], 2019.

[DW09] Marius Dadarlat and Wilhelm Winter. On the KK-theory of strongly self-absorbing C*-algebras. Math. Scand., 104(1):95-107, 2009 .

[ER78] Edward G. Effros and Jonathan Rosenberg. C*-algebras with approximately inner flip. Pacific J. Math., 77(2):417-443, 1978.

[ERS11] George A. Elliott, Leonel Robert, and Luis Santiago. The cone of lower semicontinuous traces on a $\mathrm{C}^{*}$-algebra. Amer. J. Math., 133(4):969-1005, 2011.

[Fac82] Thierry Fack. Finite sums of commutators in $\mathrm{C}^{*}$-algebras. Ann. Inst. Fourier (Grenoble), 32(1):vii, 129-137, 1982.

[FHRT17] Ilijas Farah, Bradd Hart, Mikael Rørdam, and Aaron Tikuisis. Relative commutants of strongly self-absorbing $\mathrm{C}^{*}$-algebras. Selecta Math. (N.S.), 23(1):363-387, 2017.

[Gab18] James Gabe. A new proof of Kirchberg's $\mathcal{O}_{2}$-stable classification. Journal für die reine und angewandte Mathematik, 2018.

[Gha19] Saeed Ghasemi. Strongly self-absorbing C*-algebras and Fraïssé limits. arxiv:1910.13590 [math.OA], 2019.

[GLN14] Guihua Gong, Huaxin Lin, and Zhuang Niu. Classification of finite simple amenable $\mathcal{Z}$-stable $\mathrm{C}^{*}$-algebras. arXiv:1501.00135 [math.OA], 2014.

[Jia97] Xinhui Jiang. Nonstable K-theory for $\mathcal{Z}$-stable $\mathrm{C}^{*}$-algebras. math/9707228, 1997.

[JS99] Xinhui Jiang and Hongbing Su. On a simple unital projectionless C*-algebra. Amer. J. Math., 121(2):359-413, 1999.

[JW14] Bhishan Jacelon and Wilhelm Winter. $\mathcal{Z}$ is universal. J. Noncommut. Geom., 8(4):1023-1042, 2014. 
[Kir06] Eberhard Kirchberg. Central sequences in $\mathrm{C}^{*}$-algebras and strongly purely infinite algebras. In Operator Algebras: The Abel Symposium 2004, volume 1 of Abel Symp., pages 175-231. Springer, Berlin, 2006.

[KR14] Eberhard Kirchberg and Mikael Rørdam. Central sequence C*algebras and tensorial absorption of the Jiang-Su algebra. J. Reine Angew. Math., 695:175-214, 2014.

[Lin17] Huaxin Lin. From the basic homotopy lemma to the classification of $\mathrm{C}^{*}$-algebras, volume 124 of CBMS Regional Conference Series in Mathematics. Published for the Conference Board of the Mathematical Sciences, Washington, DC; by the American Mathematical Society, Providence, RI, 2017.

[Lor93] Terry A. Loring. $C^{*}$-algebras generated by stable relations. $J$. Funct. Anal., 112(1):159-203, 1993.

[Naw13] Norio Nawata. Picard groups of certain stably projectionless $\mathrm{C}^{*}$ algebras. J. Lond. Math. Soc. (2), 88(1):161-180, 2013.

[NR16] Ping Wong Ng and Leonel Robert. Sums of commutators in pure C*-algebras. Münster J. Math., 9(1):121-154, 2016.

[Oza13] Narutaka Ozawa. Dixmier approximation and symmetric amenability for $\mathrm{C}^{*}$-algebras. J. Math. Sci. Univ. Tokyo, 20(3):349-374, 2013.

[Pet85] Dénes Petz. Spectral scale of self-adjoint operators and trace inequalities. Journal of Mathematical Analysis and Applications, 109(1):74-82, 1985 .

[RLL00] Mikael Rørdam, Flemming Larsen, and Niels Laustsen. An introduction to K-theory for $\mathrm{C}^{*}$-algebras, volume 49 of London Mathematical Society Student Texts. Cambridge University Press, Cambridge, 2000.

[Rob12] Leonel Robert. Classification of inductive limits of 1-dimensional NCCW complexes. Adv. Math., 231(5):2802-2836, 2012. 
[Rør92] Mikael Rørdam. On the structure of simple $\mathrm{C}^{*}$-algebras tensored with a UHF-algebra. II. J. Funct. Anal., 107(2):255-269, 1992.

[Rør02] Mikael Rørdam. Classification of nuclear, simple $\mathrm{C}^{*}$-algebras. In Classification of nuclear $\mathrm{C}^{*}$-algebras. Entropy in operator algebras, volume 126 of Encyclopaedia Math. Sci., pages 1-145. Springer, Berlin, 2002.

[Rør04] Mikael Rørdam. The stable and the real rank of $\mathcal{Z}$-absorbing C*-algebras. Internat. J. Math., 15(10):1065-1084, 2004.

[RS87] Jonathan Rosenberg and Claude Schochet. The Künneth theorem and the universal coefficient theorem for Kasparov's generalized K-functor. Duke Math. J., 55(2):431-474, 1987.

[RS10] Leonel Robert and Luis Santiago. Classification of *-homomorphisms from $C_{0}(0,1]$ to a $\mathrm{C}^{*}$-algebra. J. Funct. Anal., 258(3):869-892, 2010.

[RW10] Mikael Rørdam and Wilhelm Winter. The Jiang-Su algebra revisited. J. Reine Angew. Math., 642:129-155, 2010.

[Sat10] Yasuhiko Sato. The Rohlin property for automorphisms of the Jiang-Su algebra. J. Funct. Anal., 259(2):453-476, 2010.

[Sch18] Christopher Schafhauser. Subalgebras of simple AF-algebras. arXiv:1807.07381 [math.OA], Jul 2018.

[Sko16] Paul Skoufranis. Closed convex hulls of unitary orbits in $\mathrm{C}^{*}$ algebras of real rank zero. J. Funct. Anal., 270(4):1319-1360, 2016.

[Tik16] Aaron Tikuisis. K-theoretic characterization of $\mathrm{C}^{*}$-algebras with approximately inner flip. Int. Math. Res. Not. IMRN, (18):56705694, 2016.

[TW07] Andrew S. Toms and Wilhelm Winter. Strongly self-absorbing C*-algebras. Trans. Amer. Math. Soc., 359(8):3999-4029, 2007.

[TW08] Andrew S. Toms and Wilhelm Winter. $\mathcal{Z}$-stable ASH algebras. Canad. J. Math., 60(3):703-720, 2008. 
[TWW17] Aaron Tikuisis, Stuart White, and Wilhelm Winter. Quasidiagonality of nuclear $\mathrm{C}^{*}$-algebras. Ann. of Math. (2), 185(1):229-284, 2017.

[Win09] Wilhelm Winter. Covering dimension for nuclear $\mathrm{C}^{*}$-algebras. II. Trans. Amer. Math. Soc., 361(8):4143-4167, 2009.

[Win11] Wilhelm Winter. Strongly self-absorbing $\mathrm{C}^{*}$-algebras are $\mathcal{Z}$ stable. J. Noncommut. Geom., 5(2):253-264, 2011.

[Win12] Wilhelm Winter. Nuclear dimension and $\mathcal{Z}$-stability of pure $\mathrm{C}^{*}$ algebras. Invent. Math., 187(2):259-342, 2012.

[Win14] Wilhelm Winter. Localizing the Elliott conjecture at strongly self-absorbing C*-algebras. J. Reine Angew. Math., 692:193-231, 2014.

[WZ09] Wilhelm Winter and Joachim Zacharias. Completely positive maps of order zero. Münster J. Math., 2:311-324, 2009.

[WZ10] Wilhelm Winter and Joachim Zacharias. The nuclear dimension of C*-algebras. Adv. Math., 224(2):461-498, 2010. 

